\title{
Algumas propriedades do espaço topológico dos irracionais
}

\author{
Henry Naranjo Teheran
}

\author{
DisSERTAÇÃO APRESENTADA \\ $\mathrm{AO}$ \\ Instituto de MATEMÁticA E EstatísticA \\ DA \\ Universidade DE SÃO PAUlo \\ PARA
OBTENÇÁTO DO TÍTULO \\ $\mathrm{DE}$ \\ Mestre EM CiênCIAS
}

\author{
Área de Concentração: Matemática \\ Orientadora: Profa. Dra. Ofelia Teresa Alas
}

Durante o desenvolvimento deste trabalho o autor recebeu auxílio financeiro do CNPq.

São Paulo, 29 de setembro de 2011. 


\section{Algumas propriedades do espaço topológico dos irracionais}

Este exemplar corresponde à redação final da dissertação devidamente corrigida e defendida por Henry Naranjo Teheran e aprovada pela Comissão Julgadora.

Banca Examinadora:

- Profa. Dra. Ofelia Teresa Alas (orientadora) - IME-USP.

- Profa. Dra. Lucia Renato Junqueira - IME-USP.

- Prof. Dr. Marcelo Dias Passos - UNIFESP. 


\section{Agradecimentos}

Agradeço primeiramente a Deus por dar-me força espiritual e segurança ao longo de minha estadia no Brasil.

À minha família, principalmente aos meus pais Hernando e Luz María, pelo apoio e educação que sempre recebi deles.

À minha professora orientadora Ofelia T. Alas pela paciência, ensinamentos e bons conselhos. Serei sempre grato a ela.

Ao IME-USP pela imensa oportunidade em estar aqui.

Ao Hector, à Nubia e ao Rafael pela ajuda ao chegar nesta grande cidade para estudar no IME e na adaptação nos meus primeiros dias.

Aos meus amigos Cleber, por ter me ajudado a corrigir meus erros de português, Gustavo, por me auxiliar nas questões burocráticas do instituto. Ao Ronald, Luis, Fabiana, Janaina e a todos os meus colegas com quem partilhei a sala de estudo.

Ao CNPq pelo apoio financeiro.

À minha namorada Charytyn, pelo apoio, atenção e carinho...

Gracias.... 


\section{Resumo}

Neste trabalho estudamos algumas propriedades de $\mathbb{R} \backslash \mathbb{Q}$, visto como subespaço topológico de $\mathbb{R}$. Uma caracterização topológica é enunciada e como consequência obtemos que $\mathbb{R} \backslash \mathbb{Q}$ é homeomorfo ao $\omega^{\omega}$ com a topologia produto de Tychonoff ( $\omega$ com a topologia discreta). Também são vistos resultados sobre o espaço de Cantor que é um subespaço importante de $\mathbb{R} \backslash \mathbb{Q}$.

Em $Z F C$ (sistema axiomático de Zermelo-Fraenkel com o Axioma da Escolha) ou em $Z F C$ com algum outro axioma (por exemplo, Hipótese do Contínuo, Axioma de Martin, igualdades entre pequenos cardinais) são exibidos exemplos de espaços topológicos normais e espaços normais de Lindelöf, cujo produto com $\mathbb{R} \backslash \mathbb{Q}$ não é normal. Usando a noção de $P(2)$-espaço de K. Morita, mostra-se que para todo $P(2)$ espaço normal $X$, vale que $X \times(\mathbb{R} \backslash \mathbb{Q})$ é normal. Além disso, temos que se $X$ é um espaço normal tal que $X \times S$ é normal, para todo subespaço $S$ de $\mathbb{R} \backslash \mathbb{Q}$, então $X$ é um $P(2)$-espaço. 


\section{Abstract}

In this work we study some properties of $\mathbb{R} \backslash \mathbb{Q}$ seen as topological subspace of $\mathbb{R}$. A topological characterization is given and as a consequence, we obtain that $\mathbb{R} \backslash \mathbb{Q}$ is homeomorphic to $\omega^{\omega}$ with the Tychonoff product topology ( $\omega$ with the discrete topology). We also study the Cantor space which is an important subspace of $\mathbb{R} \backslash \mathbb{Q}$.

In $Z F C$ (Zermelo-Fraenkel axiomatic system with the Axiom of Choice) or $Z F C$ with some other axiom (for example, Continuum Hypothesis, Martin's Axiom, equalities between small cardinals) it is shown examples of normal or Lindelöf normal topological spaces such that the product with $\mathbb{R} \backslash \mathbb{Q}$ is nonnormal. Using K. Morita's $P(2)$ space, it is shown that for all normal $P(2)$-space $X$, the product $X \times(\mathbb{R} \backslash \mathbb{Q})$ is normal. Besides, we have that if $X$ is a normal space such that $X \times S$ is normal, for all subspace $S$ of $\mathbb{R} \backslash \mathbb{Q}$, then $X$ is a $P(2)$-space. 


\section{Sumário}

Lista de Símbolos

Introdução

0 $\quad$ Preliminares 5

0.1 Teoria dos Conjuntos . . . . . . . . . . . . . . . . . 5

0.2 Topologia Geral . . . . . . . . . . . . . . . . . . . . . . . . 11

0.3 Outros Resultados . . . . . . . . . . . . . . . . . . . . . 19

1 Caracterização de $\mathbb{R} \backslash \mathbb{Q}$ e de $\omega^{\omega} \quad 24$

2 Não normalidade de produtos de espaços normais por $\mathbb{R} \backslash \mathbb{Q} \quad 45$

2.1 Reta de Michael . . . . . . . . . . . . . . . . . . . . 46

$2.2 \mathrm{CH}, \mathrm{MA}$ e produto de espaços de Lindelöf com os irracionais . . . . . . 49

2.3 Concentração e produto de espaços de Lindelöf com os irracionais . . . 64

3 Produtos com um fator Lindelöf e um fator métrico separável $\quad 78$

3.1 Teorema de Rudin e Starbird . . . . . . . . . . . . . . . . . . . 79

3.2 Normalidade no produto de espaços normais com um espaço métrico separável . . . . . . . . . . . . . . . . . . 86

$\begin{array}{lr}\text { Apêndices } & 88\end{array}$

$\begin{array}{lr}\text { A Produto com um fator compacto } & 89\end{array}$

$\begin{array}{ll}\text { B Mais resultados usados no trabalho } & 100\end{array}$

$\begin{array}{ll}\text { Referências Bibliográficas } & 102\end{array}$ 


\section{Lista de Símbolos}

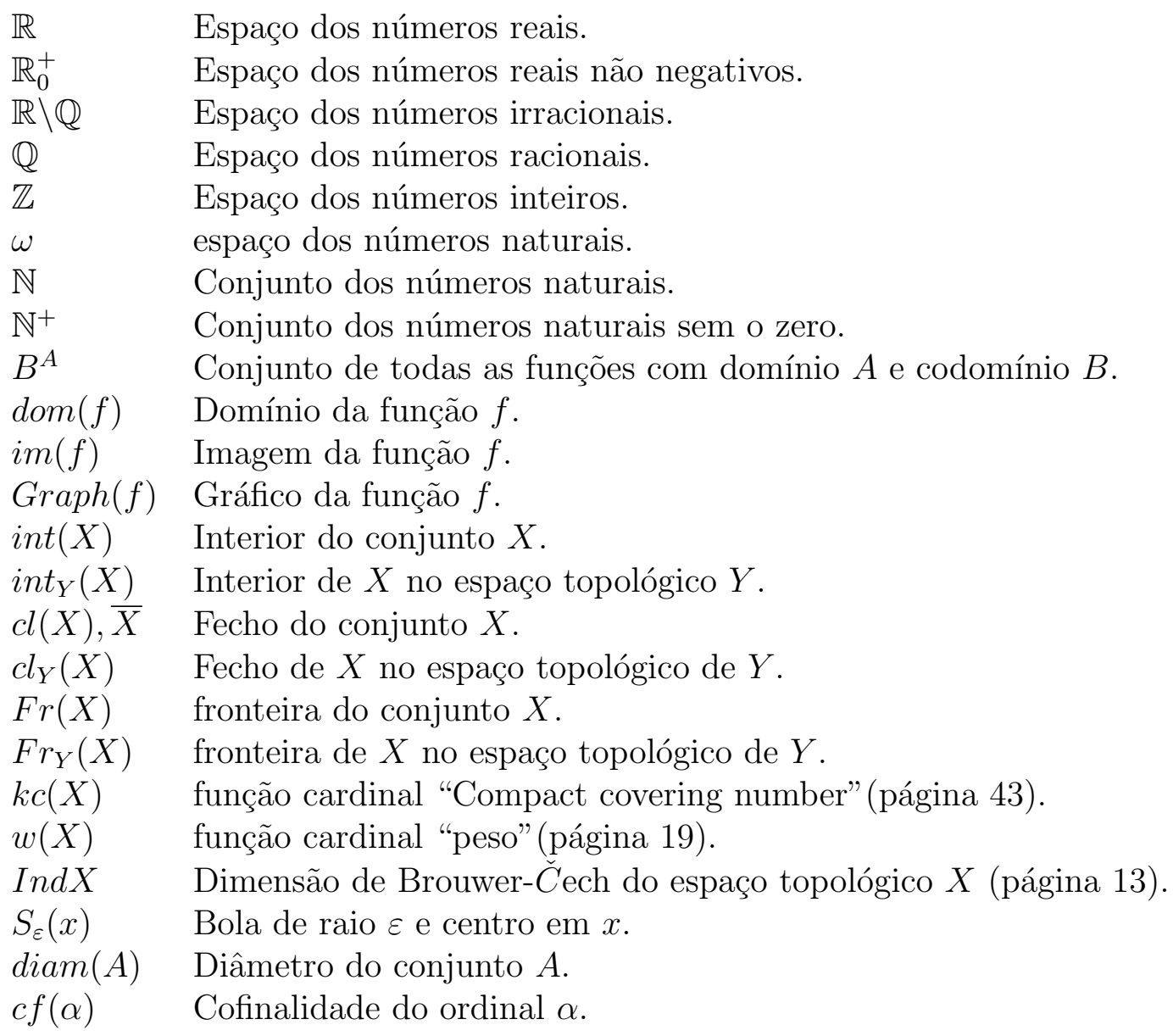




\section{Introdução}

Denotaremos por $Z F C$ o sistema axiomático de Zermelo-Fraenkel com o Axioma da Escolha.

Nesta dissertação vamos estudar algumas propriedades do espaço topológico dos números irracionais, como subespaço da reta real $\mathbb{R}$. Esse subespaço será denotado por $\mathbb{R} \backslash \mathbb{Q}$. Poderíamos pensar que $\mathbb{R} \backslash \mathbb{Q}$ é um subespaço simples de $\mathbb{R}$, mas este espaço tem propriedades muito interessantes e ainda hoje há problemas em aberto que envolvem $\mathbb{R} \backslash \mathbb{Q}$.

O subespaço $\mathbb{R} \backslash \mathbb{Q}$ é não vazio, completamente metrizável, 0-dimensional, com base de abertos enumerável e sem compactos abertos não vazios. A menos de homeomorfismos, é único com essas condições (Corolário 1.7).

Em Topologia Geral, sabe-se que o produto de espaços normais nem sempre é normal, mesmo que um dos fatores seja um espaço métrico separável. Em 1955, M. E. Rudin ([17]) exibiu, não em $Z F C$, um espaço normal $X$ cujo produto com o subespaço $[0,1]$ de $\mathbb{R}$ não é normal, utilizando uma reta de Souslin. Posteriormente, em 1971 ela construiu um exemplo em $Z F C$.

Em 1976, K. Morita, propôs a seguinte conjectura:

"Se $Y$ é um espaço de Hausdorff tal que $X \times Y$ é normal para todo espaço normal de Hausdorff $X$, então $Y$ é discreto".

Novamente M. E. Rudin, em 1978 ([18]) demonstrou a veracidade da conjectura. 
Vejamos agora, o que ocorre com a normalidade do produto quando um dos fatores é $\mathbb{R} \backslash \mathbb{Q}$. L. B. Lawrence [10, prop. 1] afirma que:

"Se $X$ e $Y$ são espaços topológicos e $Y$ é completamente metrizável, separável e não $\sigma$-compacto, então $X \times Y$ é normal se e só se $X \times(\mathbb{R} \backslash \mathbb{Q})$ é normal".

Esse resultado justifica o interesse em encontrar exemplos de espaços topológicos $X$ tais que $X \times(\mathbb{R} \backslash \mathbb{Q})$ não seja normal. Outro resultado importante, mostrado nesta dissertação (Teorema 3.4), é que, para um espaço topológico $X$ regular e de Lindelöf, $X \times(\mathbb{R} \backslash \mathbb{Q})$ é de Lindelöf, se e somente se, $X \times(\mathbb{R} \backslash \mathbb{Q})$ é normal.

Em 1963, E. A. Michael ([11]) deu o primeiro exemplo em $Z F C$ de um espaço normal cujo produto topológico com $\mathbb{R} \backslash \mathbb{Q}$ não é normal. O espaço em questão não é de Lindelöf. A questão:

"Existe em $Z F C$ um espaço topológico normal e de Lindelöf cujo produto com $\mathbb{R} \backslash \mathbb{Q}$ não é normal?"

ainda permanece em aberto.

Entretanto, adicionando outro axioma a $Z F C$ é possível obter exemplos de tais espaços (por exemplo a Hipótese do Contínuo, ou o Axioma de Martin ou a asserção $b=\omega_{1}$, etc.)

A dissertação começa com um Capítulo 0 de preliminares, onde recordamos algumas noções de Teoria dos Conjuntos e Topologia, necessárias para o desenvolvimento do trabalho.

O Capítulo 1 é dedicado ao subespaço topológico $\mathbb{R} \backslash \mathbb{Q}$. Vamos caracterizá-lo e concluir que $\mathbb{R} \backslash \mathbb{Q}$ é homeomorfo ao $\omega^{\omega}$. Também caracterizaremos o espaço de Cantor $\{0,1\}^{\omega}$ e o dos racionais $\mathbb{Q}$. Serão mencionados alguns outros resultados relacionados com $\mathbb{R} \backslash \mathbb{Q}$ e o subespaço $\{0,1\}^{\omega}$; por exemplo, veremos que todo espaço metrizável e compacto é imagem contínua do espaço de Cantor e que todo espaço metrizável, separável, 0-dimensional e que não contem compactos abertos não vazios pode ser den- 
samente imerso em $\mathbb{R} \backslash \mathbb{Q}$.

No Capítulo 2 apresentamos alguns exemplos, em $Z F C$ ou $Z F C$ com algum outro axioma adicional (ou a Hipótese do Contínuo, ou o Axioma de Martin ou $\mathfrak{b}=\omega_{1}$ ) de espaços topológicos normais para os quais o seu produto com $\mathbb{R} \backslash \mathbb{Q}$ não é normal.

No caso $Z F C$, exibimos $X=[0,1]$ como subespaço da reta de Michael (Teorema $2.1)$; isto é, $[0,1]$ com a topologia

$$
\Theta=\{U \cup V: U \text { é aberto usual em }[0,1], V \subseteq[0,1] \backslash \mathbb{Q}\}
$$

No caso $Z F C$ com mais algum axioma, o espaço topológico $X$, além de ser normal, será de Lindelöf. Nestas construções, ao invés de $\mathbb{R} \backslash \mathbb{Q}$, usaremos o seu homeomorfo $\omega^{\omega}$.

No Capítulo 3 estudamos a noção de $P(2)$-espaço para concluir que nesses espaços topológicos o produto com $\mathbb{R} \backslash \mathbb{Q}$ é sempre normal; Morita em [14] mostrou que se $X$ é um espaço topológico, o produto $X \times Y$ é normal para todo espaço metrizável e separável $Y$ se e somente se $X$ é um $P(2)$-espaço normal.

Também apresentaremos um teorema de Rudin e Starbird (Teorema 3.4) a fim de concluir que para todo espaço topológico regular e de Lindelöf $X$ temos que $X \times(\mathbb{R} \backslash \mathbb{Q})$ é normal se e somente se $X \times(\mathbb{R} \backslash \mathbb{Q})$ é de Lindelöf. Este resultado é usado no Capítulo 2 para demonstrar a normalidade ou a não normalidade do produto de alguns espaços.

Finalmente, nos Apêndices A e B exibimos alguns resultados dados por T. Przymusiński em [15], sobre o produto topológico onde um dos fatores é compacto, que nos permitem demonstrar o Corolário 3.3 e o Teorema de Rudin e Starbird mencionado acima. Também mostramos algumas proposições que são usadas no texto. Isto é feito para torná-lo mais abrangente e simplificar a leitura do mesmo.

Como um comentário final, destacamos que muitas das demonstrações nesta dissertação foram feitas tentando simplificar as apresentadas nos artigos. Algumas delas foram obtidas usando ideias de vários artigos. 


\section{Capítulo 0}

\section{Preliminares}

Neste capítulo vamos recordar algumas noções básicas e necessárias para o desenvolvimento deste trabalho. Se for necessário aprofundar em algumas destas noções veja-se [9] (no caso de Teoria dos Conjuntos) e [6] (no caso de Topologia).

\subsection{Teoria dos Conjuntos}

Usaremos o sistema axiomático de Zermelo-Fraenkel com o Axioma da Escolha (ZFC).

Operações de conjuntos e noções básicas serão supostas conhecidas (tais como: relações, ordem total e parcial, boa-ordem, isomorfismo entre conjuntos bem ordenados, elemento maximal e minimal, máximos e mínimos, extremos superiores e inferiores). O conjunto das partes de um conjunto $A$ será denotado por $P(A)$.

0.1 (Conjunto transitivo, ordinal). Um conjunto $A$ é dito transitivo se todo elemento de A é também subconjunto de A. Um conjunto é um ordinal se for transitivo e bem ordenado pela relação $\in$.

Notemos que todo elemento de um ordinal é ordinal. Em geral os ordinais serão denotados por letras minúsculas gregas: $\alpha, \beta, \gamma, \delta, \ldots$ Também é comum escrever $\alpha<\beta$ para dizer $\alpha \in \beta$. 
Vamos supor conhecidos também os conceitos de ordinal sucessor e ordinal limite. Todo conjunto não vazio $A$ de ordinais admite um supremo, $\sup (A)=\bigcup A$, e no caso de ser $A \neq \phi$ também admite um mínimo, $\bigcap A$.

Processos de indução transfinita e recursão serão feitos sobre subconjuntos bem ordenados.

0.2 (Equipotência, Ordinal Cardinal). Dois conjuntos $A$ e B são chamados equipotentes ( $e$ denotamos por $A \approx B$ ) se $A=B=\phi$ ou se $A \neq \phi \neq B$ e existir uma função $f: A \longrightarrow B$ bijetora.

Sob o Axioma de Escolha $(A C)$ todo conjunto pode ser bem ordenado. Definimos a cardinalidade de um conjunto $A$ como sendo $|A|=\min \{\alpha: \alpha$ é ordinal $e \alpha \approx A\}$. Um ordinal $\alpha$ é dito cardinal se $|\alpha|=\alpha$.

Se $A$ é um conjunto e $\lambda$ é um cardinal, definimos

$$
[A]^{<\lambda}=\{X \in P(A):|A|<\lambda\}
$$

Em particular $[\omega]^{<\omega}$ denota o conjunto de todos os subconjuntos finitos de $\omega$.

É bem conhecido que para todo conjunto $A,|A|<|P(A)|$. $\operatorname{Logo} \omega<2^{\omega}\left(\right.$ Se $\omega_{1}$ denota o menor ordinal não enumerável, então $\omega_{1} \leq 2^{\omega}$ ). A Hipótese do Contínuo (CH) é a asserção $2^{\omega}=\omega_{1}$.

0.3 (Cofinalidade de Ordinais). Sejam $\alpha, \beta$ ordinais. Se $\beta$ é ordinal sucessor $(\beta=\eta+1)$, uma função $f: \alpha \longrightarrow \beta$ é dita cofinal em $\beta$ se $\eta \in i m(f)$. Se $\beta$ é ordinal limite, então $f: \alpha \longrightarrow \beta$ é dita cofinal em $\beta$ se im $(f)$ é ilimitada em $\beta$. A cofinalidade de um ordinal $\beta$ (que denotaremos por cf $(\beta)$ ) é o menor ordinal $\alpha$ para o qual existe $f: \alpha \longrightarrow \beta$ cofinal em $\beta$. Se $\beta$ é ordinal sucessor, então $c f(\beta)=1$. Logo só há interesse nas cofinalidades de ordinais limites. Se $\beta$ é limite e cf $(\beta)=\alpha$, podemos provar que existe $f: \alpha \longrightarrow \beta$ cofinal em $\beta$ estritamente crescente. 
0.4 (Pré-ordem). Uma relação $\preceq$ em um conjunto $P$ é uma pré-ordem se $\preceq$ é reflexiva e transitiva. Nestas condições dizemos que $(P, \preceq)$ é um conjunto pré-ordenado. Como é costume, abusaremos da notação ao escrever $P$ ao invés de $(P, \preceq)$, quando a definição da pré-ordem $\preceq$ seja clara dentro do contexto.

Vamos definir agora a pré-ordem $\leq_{*}$ em $\omega^{\omega}$

$0.5\left(\leq_{*} \mathrm{e}<_{*}\right)$. Sejam $x, y \in \omega^{\omega}$, dizemos que $x<_{*} y$ (ou respectivamente $x \leq_{*} y$ ) se existe $m \in \mathbb{N}$, tal que $x(j)<y(j)$ (ou respectivamente $x(j) \leq_{*} y(j)$ ) para todo $j$, tal que $m<j$. a

Dizemos que uma família $B \subseteq \omega^{\omega}$ é uma família ilimitada se for ilimitada em $\left(\omega^{\omega}, \leq_{*}\right)$, isto é, se para cada $f \in \omega^{\omega}$ existe $g \in B$ tal que $g \Varangle_{*} f . D \subseteq \omega^{\omega}$ é dita uma família dominante se for cofinal em $\left(\omega^{\omega}, \leq_{*}\right)$, isto é, se para cada $f \in \omega^{\omega}$ existe $g \in D$ tal que $f \leq_{*} g$.

0.6 (Cardinais $\mathfrak{b}$ e $\mathfrak{d})$. Definimos:

- $\mathfrak{b}=\min \left\{|B|: B \subseteq \omega^{\omega}\right.$ é uma familia ilimitada em $\left.\left(\omega^{\omega}, \leq_{*}\right)\right\}$

- $\mathfrak{d}=\min \left\{|D|: D \subseteq \omega^{\omega}\right.$ é uma família dominante em $\left.\left(\omega^{\omega}, \leq_{*}\right)\right\}$

Temos claramente que $\mathfrak{b} \leq \mathfrak{d}$ (pois toda família dominante é ilimitada)

Lema 0.1. Vale que $\omega_{1} \leq \mathfrak{b} \leq \mathfrak{c}$ e que $\omega_{1} \leq \mathfrak{d} \leq \mathfrak{c} .\left(\omega_{1} \leq \mathfrak{b} \leq \mathfrak{d} \leq \mathfrak{c}\right)$

Dem: Mostremos que uma família enumerável qualquer de $\omega^{\omega}$ não pode ser ilimitada. Seja $B=\left\{f_{n}: n<\omega\right\}$ uma família de funções de $\omega$ em $\omega$. Consideremos $g: \omega \longrightarrow \omega$ dada por $g(n)=\max \left\{f_{i}(j): i, j \leq n\right\}+1$. Seja $f_{m} \in B$ :

- Se $m=0$ então $f_{m} \leq g$ e assim $f_{m} \leq_{*} g$.

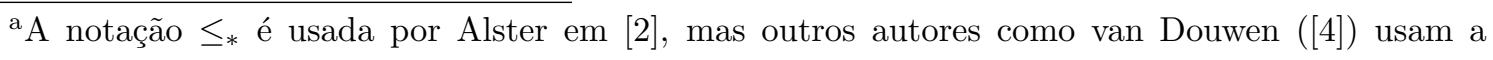
notação $\leq *$. 
- Se $m \neq 0$ então $\left\{n<\omega: g(n)<f_{m}(n)\right\} \subseteq\{0,1, \ldots, m-1\}$, logo existe $m<\omega$ tal que se $m \leq k$ temos que $f_{m}(k) \leq g(k)$. Portanto $f_{m} \leq_{*} g$.

Concluímos que $\omega_{1} \leq \mathfrak{b}$. Além disso segue-se também que $\omega_{1} \leq \mathfrak{d}$ já que uma família enumerável não pode ser dominante pois nesse caso seria também ilimitada.

Vamos ver agora algumas definições alternativas para os pequenos cardinais $\mathfrak{b}$ e $\mathfrak{d}$ que serão úteis no desenvolvimento do trabalho.

Na definição 0.5, foram definidas as relações $\leq_{*} \mathrm{e}<_{*}$ em $\omega^{\omega}$. Vamos introduzir uma nova relação $<^{*}$, em $\omega^{\omega}$, dada por

$$
\text { " } x<^{*} y \text { se e só se } x \leq_{*} y \text { e } y \Varangle_{*} x " .
$$

Notemos que nestas condições " $x<^{*} y$ " é mais que pedir as condições " $x \leq_{*} y$ e $x \neq y^{\prime \prime}$, pois como não vale a antisimetria, podemos encontrar $x, y \in \omega^{\omega}$ tais que $x \neq y$, $x \leq_{*} y$ e $y \leq_{*} x$. As relações $<_{*} \mathrm{e}<^{*}$ são bem diferentes logo devemos ter cuidado de não confundi-las, pois se $x<_{*} y$, então $x<^{*} y$ mas nem sempre vale a recíproca.

0.7. Definimos os seguintes cardinais:

- $\mathfrak{b}_{1}=\min \left\{|B|: B\right.$ é um subconjunto ilimitado de $\omega^{\omega}$ de funções estritamente crescentes e que é bem ordenado por $\left.<^{*}\right\}$

- $\mathfrak{d}_{1}=\min \left\{|D|: D\right.$ é cofinal em $\left.\left(\omega^{\omega}, \leq\right)\right\}$

Teorema 0.2. Valem $\mathfrak{b}=\mathfrak{b}_{1}$ e $\mathfrak{d}=\mathfrak{d}_{1}$.

Dem: Vamos mostrar aqui que $\mathfrak{b}=\mathfrak{b}_{1}$. Para a prova de $\mathfrak{d}=\mathfrak{d}_{1}$ veja-se $[4$, teorema 3.6.].

$\mathfrak{b}=\mathfrak{b}_{1}$. A desigualdade $\mathfrak{b} \leq \mathfrak{b}_{1}$ é clara. Para mostrar a outra desigualdade consideremos $F=\left\{f_{\beta} \in \omega^{\omega}: \beta<\mathfrak{b}\right\}$ como sendo um subconjunto ilimitado de $\omega^{\omega}$ e consideremos também $G=\left\{f \in \omega^{\omega}: f\right.$ é estritamente crescente $\}$. Claramente $G$ é dominante. 
Vamos construir por recursão transfinita uma família $\left\{g_{\beta}: \beta<\mathfrak{b}\right\} \subseteq G$ tal que para cada $\beta<\mathfrak{b}$ vale a seguinte asserção

$$
\left(\forall f \in\left\{g_{\varepsilon}: \varepsilon<\beta\right\} \cup\left\{f_{\beta}\right\}\right)\left(f<^{*} g_{\beta}\right)
$$

Escolhemos $g_{0} \in G$ tal que $f_{0}<^{*} g_{0}$. Para $0<\beta<\mathfrak{b}$ supomos determinadas as funções $g_{\varepsilon}$ satisfazendo (0.1.1) para cada $\varepsilon<\beta$. Da definição de $\mathfrak{b}$ e sabendo que $\beta<\mathfrak{b}$, temos que o conjunto $V_{\beta}=\left\{g_{\varepsilon}: \varepsilon<\beta\right\} \cup\left\{f_{\beta}\right\}$ é limitado; isto é, existe $g \in \omega^{\omega}$ tal que para cada $f \in V_{\beta}$ temos que $f \leq_{*} g$. Agora tomamos $g_{\beta} \in G$ satisfazendo

$$
(\forall n<\omega)\left(g(n)<g_{\beta}(n)\right)
$$

então $g<^{*} g_{\beta}$. Logo vale (0.1.1) para $\beta$ e assim $\left\{g_{\beta}: \beta<\mathfrak{b}\right\}$ está construída. Notemos agora que um limitante superior de $\left\{g_{\beta}: \beta<\mathfrak{b}\right\}$, também seria limitante superior de $F$, $\log 0\left\{g_{\beta}: \beta<\mathfrak{b}\right\}$ é uma família ilimitada de $\omega^{\omega}$, cujos elementos são estritamente crescente e claramente bem ordenada por $<^{*}$. Daí $\mathfrak{b}_{1} \leq \mathfrak{b}$ e a igualdade está demonstrada.

\section{Axioma de Martin (MA)}

Para introduzir o Axioma de Martin vamos precisar de alguns conceitos prévios.

0.8. Seja $(P, \preceq)$ um conjunto pré-ordenado:

1. Dois elementos $p, q \in P$ são compatíveis se existir $r \in P$ tal que $r \preceq p$ e $r \preceq q$.

2. Dois elementos $p, q \in P$ são incompatíveis se não são compatíveis.

3. Um subconjunto $A \subseteq P$ é uma anticadeia se quaisquer dois elementos distintos de A são incompativeis.

4. Um subconjunto $D \subseteq P$ é denso se para qualquer $p \in P$, existe $d \in D$ tal que $d \preceq p$. 
0.9. Um conjunto pré-ordenado satisfaz a condição da cadeia enumerável (c.c.c.), se toda anticadeia é enumerável.

Exemplo 0.1. Seja $(X, \tau)$ um espaço topológico e

$$
P=\{p \subseteq X: p \neq \phi, p \text { é aberto em } X\}
$$

com a pré-ordem $\preceq$ dada pela inclusão de conjuntos ( $p \preceq q$ se e só se $p \subseteq q$ ). Então $p, q \in P$ são incompatíveis se e só se $p \cap q=\phi$.

Dizemos que um espaço topológico $(X, \tau)$ tem celularidade enumerável ou satisfaz c.c.c. se todo subconjunto de $\tau$, cujos elementos são dois a dois disjuntos, é enumerável.

0.10. Se $D$ é um subconjunto denso de um conjunto pré-ordenado $P$, então um subconjunto $G$ de $P$ é dito filtro $D$-genérico em $P$ se satisfaz as seguintes condições:

1. Se $a \in G, b \in P$ e $a \preceq b$, então $b \in G$.

2. Se $a, b \in G$, então existe $c \in G$ tal que $c \preceq a$ e $c \preceq b$.

3. $D \cap G \neq \phi$.

$S e \mathcal{D}$ é uma família de conjuntos densos em $P$, então $G$ é um filtro $\mathcal{D}$-genérico em $P$ se é um filtro $D$-genérico para cada $D \in \mathcal{D}$.

Lema 0.3. Se $(P, \preceq)$ é um conjunto pré-ordenado e $\mathcal{D}$ é uma família não vazia enumerável de subconjuntos densos de $P$, então existe um filtro $\mathcal{D}$-genérico em $P$. De fato, para cada $p \in P$, existe um filtro $\mathcal{D}$-genérico $G$ em $P$ tal que $p \in G$.

Dem: Suponhamos que $\mathcal{D}=\left\{D_{1}, D_{2}, \ldots\right\}$ é uma família enumerável de subconjuntos densos em $P$. Tomemos $p \in P$ um elemento qualquer e definamos por indução o 
conjunto $\left\{p_{n} \in P: n \in \mathbb{N}\right\}$ da seguinte forma:

$p_{0}=p$ e, para cada $n>0$, tomamos $p_{n} \in D_{n}$ tal que $p_{n} \preceq p_{n-1}$.

Vejamos que o conjunto

$$
G=\left\{x \in P: \text { existe } n \in \mathbb{N} \text { tal que } p_{n} \preceq x\right\}
$$

é um filtro $\mathcal{D}$-genérico em $\mathrm{P}$ e que $p \in G$. De fato, é claro que $p \in G$. Agora se $r \in P$, $q \in G$ e $q \preceq r$, então $r \in G$, pois existe $n \in \mathbb{N}$ tal que $p_{n} \preceq q$ (pois $q \in G$ ).

Se $q_{1}, q_{2} \in G$ existem $n, m \in \mathbb{N}$ tais que $p_{m} \preceq q_{1}$ e $p_{n} \preceq q_{2}$. Sem perda de generalidade, suponhamos que $m \leq n$. Logo $p_{n} \preceq q_{1}$ e $p_{n} \preceq q_{2}$.

Finalmente é facil ver que $G \cap D_{n} \neq \phi$ para cada $n \in \mathbb{N}$.

0.11. $\mathbf{M A}_{\kappa}$ é a afirmação:

Se $\kappa$ é um cardinal infinito, $(P, \preceq)$ é um conjunto pré-ordenado que satisfaz c.c.c. $e$ $\mathcal{D}$ é uma família de subconjuntos densos em $P$, com $|\mathcal{D}| \leq \kappa$, então existe um filtro $\mathcal{D}$-genérico em $P$.

Notemos que $\mathbf{M A}_{\aleph_{0}}$ é justamente o Lema 0.3. O Axioma de Martin afirma que $\mathbf{M A}_{\kappa}$ vale para cada $\kappa<2^{\aleph_{0}} ;$ isto é,

0.12. O Axioma de Martin [MA] é a afirmação:

Se $(P, \preceq)$ é um conjunto pré-ordenado que satisfaz c.c.c. e se $\mathcal{D}$ é uma coleção de menos que $2^{\aleph_{0}}$ subconjuntos densos de $P$, então existe um filtro $\mathcal{D}$-genérico em $P$.

\subsection{Topologia Geral}

Nesta seção vamos assumir conhecidas algumas noções topológicas básicas, por exemplo os conceitos de espaço topológico, funções (contínuas, abertas, fechadas, homeomorfismos), ponto (interior, de aderência, de acumulação, de fronteira), vizinhança, base de um ponto e base de abertos de um espaço topológico. 
0.13 (Ponto de Condensação e Isolado). Seja X um espaço topológico, um ponto $t \in X$ é chamado ponto isolado se $\{t\}$ é aberto.

Um ponto $s \in X$ é chamado de ponto de condensação de $A \subseteq X$ se toda vizinhança $V$ de s satisfaz que $|V \cap A| \geq \omega_{1}$.

O teorema seguinte é facil de verificar.

Teorema 0.4. Todo subconjunto $T$ de um espaço metrizável e separável tal que $|T|>$ $\omega$, contem um ponto de condensação.

Vamos assumir também que são conhecidos os axiomas de enumerabilidade e de separação $\left(T_{0}, T_{1}, T_{2}, T_{3}, T_{3 \frac{1}{2}}, T_{4}\right)$. (Os espaços topológicos $T_{2}$ são chamados espaços de Hausdorff).

0.14. Seja $X$ um espaço topológico. $X$ é dito:

- Espaço Regular: se verifica os axiomas $T_{1}$ e $T_{3}$.

- Espaço Normal: se verifica os axiomas $T_{1}$ e $T_{4}$.

- Espaço Completamente regular ou de Tychonoff: se verifica os axiomas $T_{1}$ e $T_{3 \frac{1}{2}}$

Sejam $(X, \tau)$ um espaço topológico e $A \subseteq X$; a topologia de subespaço que $\tau$ induz em $A$ é $\tau \uparrow A=\{A \cap W: W \in \tau\}$. Uma propriedade é dita hereditária se é preservada para subespaços, isto é, se um espaço $X$ satisfaz a propriedade $P$ e $A \subseteq X$, então o subespaço $A$ satisfaz $P$.

Teorema 0.5. Seja $f: X \longrightarrow Y$ uma aplicação continua, fechada e sobrejetora. Se $X$ é um espaço normal, então $Y$ também é um espaço normal. 
0.15 (Espaço 0-dimensional). Um espaço topológico $X$ é dito espaço 0-dimensional se possuir uma base formada por abertos-fechados.

A seguir, introduziremos a noção de dimensão de Brouwer-Čech de um espaço topológico:

0.16 (Dimensão de Brouwer-Čech). Seja X um espaço topológico normal e $n \in \mathbb{Z}$, $n \geq-1$. Dizemos que:

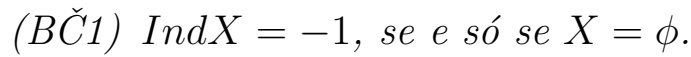

(BČ2) IndX $\leq n$, se para cada $A \subseteq X$ fechado e para cada $V \subseteq X$ aberto contendo $A$, existe um conjunto aberto $U \subseteq X$ tal que $A \subseteq U \subseteq V$ e $\operatorname{Ind} \operatorname{Fr}(U) \leq n-1$.

(BС̆з) IndX $=n$, se Ind $X \leq n$ e a desigualdade Ind $X \leq n-1$ não se verifica.

$(B \check{C} 4)$ IndX $=\infty$, se a desigualdade Ind $X \leq n$ não se verifica qualquer $n$.

O teorema seguinte relaciona os conceitos de dimensão de Brouwer-Čech e 0-dimensionalidade. Sua demonstração é consequencia da definição.

Teorema 0.6. Se $X$ é um espaço topológico normal tal que IndX $=0$, então $X$ é 0-dimensional.

0.17 (Espaços Metrizáveis). Um espaço métrico é um par $(M, d)$, onde $M$ é um conjunto e d é uma função (métrica) do conjunto $M \times M$ em $\mathbb{R}_{0}^{+}$(o conjunto dos números reais não negativos) satisfazendo as seguintes propriedades:

(M1) Para todo $x, y \in M, d(x, y)=0$ se é só se $x=y$.

(M2) Para todo $x, y \in M$ temos que $d(x, y)=d(y, x)$. 
(M3) Para todo $x, y, z \in M, d(x, y) \leq d(x, z)+d(z, y)$

Lembremos que a Propriedade (M3) é chamada desigualdade triangular.

Por abuso de notação, quando estiver claro qual é a métrica, denotaremos o espaço métrico simplesmente por $M$.

Um $\operatorname{par}(M, d)$ onde d satisfaz só (M2) e (M3) é chamado espaço pseudométrico e d é chamada pseudo-métrica sobre $M$.

Se $(M, d)$ é um espaço métrico e $\tau_{d}$ é o conjunto

$\left\{U \subseteq M\right.$ : para cada $x \in U$ existe $\varepsilon<0$ tal que $\left.S_{\varepsilon}(x) \subseteq U\right\}$

onde $S_{\varepsilon}(x)$ é a bola de centro em $x$ e raio $\varepsilon$ em $M, \tau_{d}$ é a topologia sobre $M$ associada à métrica d.

Um espaço topológico $(X, \tau)$ é dito espaço metrizável se existir sobre ele uma métrica $d$, tal que $\tau=\tau_{d}$.

0.18 (Família localmente finita e discreta). Seja $X$ um espaço topológico e $\mathcal{F}$ uma família de subconjuntos de $X$. Dizemos que $\mathcal{F}$ é localmente finita (ou, respectivamente, discreta) se para cada ponto $x \in X$ existe uma vizinhança $U$ de $x$ tal que a cardinalidade do conjunto $\{F \in \mathcal{F}: F \cap U \neq \phi\}$ é finita (ou, respectivamente, menor ou igual a 1).

Uma familia $A$ de subconjuntos de $X$ é chamada $\sigma$-localmente finita se $A=$ $\bigcup_{i<\omega} F_{i}$ onde para cada $i<\omega, F_{i}$ é uma família localmente finita. Analogamente definimos família $\sigma$-discreta.

O seguinte teorema caracteriza os espaços metrizáveis:

Teorema 0.7 (Nagata, Smirnov, Bing). Um espaço topológico $Z$, que é $T_{2}$ e $T_{3}$, é metrizável se e somente se admite uma base de abertos $\sigma$-discreta (ou $\sigma$-localmente finita). 
Se $(M, d)$ é um espaço métrico e $\phi \neq A \subseteq M$ é um subconjunto limitado de $M$, então definimos o diâmetro de $A$ (que denotaremos por $\operatorname{diam}(A))$ como sendo

$$
\operatorname{diam}(A)=\sup _{x, y \in \bar{A}} d(x, y)
$$

Por outro lado, dizemos que um sequência $\left(x_{n}\right)_{n \in \mathbb{N}}$ em $M$ é uma sequência de Cauchy se para cada $\varepsilon>0$ existir $n_{0} \in \mathbb{N}$ tal que para quaisquer $n, m \geq n_{0}$ temos $d\left(x_{n}, x_{m}\right)<\varepsilon$.

0.19 (Espaço Métrico completo). Um espaço métrico $(M, d)$ é um espaço métrico completo se toda sequência de Cauchy converge. Um espaço topológico $(X, \tau)$ é completamente metrizável se existir uma métrica d definida nele tal que $(X, d)$ é completo $e \tau_{d}=\tau$.

Vamos definir agora algumas propriedades topológicas e para isto vamos supor conhecidas as noções de cobertura (recobrimento) e subcobertura (sub-recobrimento). Vamos dizer que $\left\{W_{j}: j \in J\right\}$ é um refinamento da cobertura $\left\{U_{i}: i \in I\right\}$, se $\bigcup\left\{W_{j}: j \in J\right\}=\bigcup\left\{U_{i}: i \in I\right\}$ e para cada $j \in J$ existir $i_{j} \in I$ tal que $W_{j} \subseteq U_{i_{j}}$.

0.20 (Algumas Propriedades topológicas). Seja $(X, \tau)$ um espaço topológico:

- Compacidade: $(X, \tau)$ é dito compacto se toda cobertura por abertos tem uma subcobertura finita.

- Lindelöf: $(X, \tau)$ é dito um espaço de Lindelöf se toda cobertura por abertos de $X$ tem uma subcobertura enumerável.

- Enumeravelmente compacto: $(X, \tau)$ é dito enumeravelmente compacto se toda cobertura enumerável por abertos de X tem uma subcobertura finita.

- Paracompacidade: $(X, \tau)$ é dito um espaço paracompacto se toda cobertura por abertos de $X$ admite um refinamento aberto localmente finito. 
- Enumeravelmente paracompacto: $(X, \tau)$ é enumeravelmente paracompacto se toda cobertura enumerável por abertos de $X$ tem um refinamento aberto localmente finito.

- $\kappa$-paracompacto: Seja $\kappa$ um cardinal. $(X, \tau)$ é $\kappa$-paracompacto se toda cobertura de cardinalidade menor ou igual a $\kappa$ por abertos de $X$, admite um refinamento aberto localmente finito.

Alguns textos pedem, para que um espaço topológico seja paracompacto, enumeravelmente paracompacto ou $\kappa$-paracompacto, além do que pedimos na nossa definição, que o espaço seja $T_{2}$ (como em [6] por exemplo). Assim devemos ter um pouco de cuidado para não confundir as definições.

Teorema 0.8. Se X é um espaço metrizável, as seguintes afirmações são equivalentes:

1. X é de Lindelöf.

2. X é separável.

3. X tem base enumerável.

0.21 ( $\sigma$-compacidade). Um subconjunto $A$ de um espaço topológico $X$ é $\sigma$-compacto se ele é reunião enumerável de conjuntos compactos.

Temos agora os seguintes teoremas:

Teorema 0.9. 1. Todo espaço topológico paracompacto e $T_{2}$ é normal.

2. Todo espaço de Lindelöf e regular é paracompacto.

3. Todo espaço de Lindelöf e regular é normal. 
Teorema 0.10. Se um espaço paracompacto e de Hausdorff X contem um subespaço denso A que é de Lindelöf, então X é de Lindelöf.

A seguir apresentamos uma caracterização para os espaços enumeravelmente paracompactos.

Teorema 0.11. Para um espaço $X$ de Hausdorff as seguintes condições são equivalentes:

1. X é enumeravelmente paracompacto.

2. Para cada sequência crescente $W_{0} \subseteq W_{1} \cdots$ de subconjuntos abertos de $X$ satisfazendo $X=\bigcup\left\{W_{i}: i<\omega\right\}$, existe uma sequência $T_{0}, T_{1}, \ldots$ de subconjuntos fechados de $X$ tais que $T_{i} \subseteq W_{i}$ para cada $i<\omega$ e $X=\bigcup\left\{\operatorname{int}\left(T_{i}\right): i<\omega\right\}$.

3. Cada família $\left\{F_{i}: i<\omega\right\}$ de subconjuntos fechados em $X, \operatorname{com} \bigcap\left\{F_{i}: i<\omega\right\}=\phi$ e $F_{i+1} \subseteq F_{i}$ para cada $i<\omega$, existe uma família $\left\{U_{i}: i<\omega\right\}$ de subconjuntos abertos de $X$, tais que $X=\bigcup\left\{U_{i}: i<\omega\right\}$ e $F_{i} \cap \overline{U_{i}}=\phi$ para cada $i<\omega$.

Dem: As condições 1. e 2. são equivalentes, ver em [6, teorema 5.2.1] ( $i$ ) é equivalente a $($ iii $)$ ). Agora mostraremos que 2. é equivalente a 3 .

Para ver $3 . \Rightarrow 2$., basta tomar $F_{i}=X \backslash W_{i}$ para cada $i<\omega$, logo existem a família $\left\{U_{i}: i<\omega\right\}$ como em 3 . e tomando $T_{i}=\operatorname{cl}\left(U_{i}\right)$ para cada $i<\omega$ vale 2 .

Para mostrar 2. $\Rightarrow 3$. tomemos $W_{i}=X \backslash F_{i}$ para cada $i<\omega$. Deste modo existe a família $\left\{T_{i}: i<\omega\right\}$ como em 2 . e considerando $U_{i}=\operatorname{int}\left(T_{i}\right)$ mostra-se que vale 3 .

0.22 (Conjunto $G_{\delta}$ e conjunto $F_{\sigma}$ ). Seja $X$ um espaço topológico. $A \subseteq X$ é dito um conjunto $G_{\delta}$ se $A=\bigcap_{i \in \mathbb{N}} V_{i}$, onde $V_{i}$ é aberto em $X$ para cada $i \in \mathbb{N}$.

$A$ é dito um conjunto $F_{\sigma}$ se $A=\bigcup_{i \in \mathbb{N}} F_{i}$, onde $F_{i}$ é fechado em $X$ para cada $i \in \mathbb{N}$. 
Todo subespaço $G_{\delta}$ de um espaço métrico completo é completamente metrizável.

Agora apresentaremos um resultado devido a Tumarkin [6, pag. 422, 7.4.17].

Teorema 0.12 (Tumarkin). Para todo subespaço $X$ de um espaço metrizável $M$, existe um conjunto $G_{\delta} X^{*} \subseteq M$ tal que $X \subseteq X^{*}$ e IndX $=$ Ind $X^{*}$.

Vamos introduzir agora o conceito de espaço de Cantor e de Baire.

0.23 (Espaço de Baire:). Se X é um espaço topológico e $A \subseteq X$ é tal que o interior de seu fecho é vazio (isto é, int $(\bar{A})=\phi$ ) dizemos que $A$ é um conjunto raro. Um subconjunto de $X$ é dito magro se for reunião enumerável de conjuntos raros. Um espaço $X$ é dito um espaço de Baire se todo subconjunto magro de $X$ tem interior vazio.

Por exemplo, os espaços completamente metrizáveis são espaços de Baire.

0.24 (Espaço de Cantor). Consideremos o espaço topológico $\{0,1\}^{\omega}$ com a topologia produto de Tychonoff, onde $\{0,1\}$ tem a topologia discreta. Um espaço $X$ é dito de Cantor se existir um homeomorfismo $\Phi: X \longrightarrow\{0,1\}^{\omega}$.

0.25 (Compactificação). Um par $(Y, c)$ é uma compactificação do espaço topológico $T_{2} X$, se $Y$ é compacto, $T_{2}$ e $c: X \longrightarrow Y$ for um homeomorfismo sobre a sua imagem tal que $\overline{c[X]}=Y$. Denotaremos $(Y, c)$ por $c X$. Uma compactificação que vamos usar neste trabalho é a compactificação de Čech Stone de X, denotada por $\beta X$ (veja-se [6, seção 3.6]).

Vamos dizer que um espaço topológico $Y$ é uma compactificação métrica de um espaço $X$, se $Y$ for compactificação de $X$, como na definição anterior, e $Y$ for métrizável.

A seguir introduziremos a função cardinal chamada peso: 
0.26 (Função Cardinal Peso). Seja X um espaço topológico. Definimos o peso de $X$ como sendo:

$$
w(X)=\min \{|\mathfrak{B}|: \mathfrak{B} \text { é uma base de abertos para } X\}+\omega
$$

\subsection{Outros Resultados}

Nesta seção apresentaremos alguns resultados que envolvem Teoria dos Conjuntos e Topologia.

Uma aplicação do Axioma de Martin à Topologia (ver [9, pag. 65; teorema 3.4]) é que as seguintes asserções são equivalentes para um cardinal $\kappa$ infinito:

i. $\mathbf{M A} \mathbf{A}_{\kappa}$

ii. Num espaço topológico compacto, de Hausdorff e não vazio com celularidade enumerável, a intersecção de $\kappa$ subconjuntos densos e abertos em $X$ é não vazia.

No Teorema 0.13 , que apresentaremos a seguir, mostraremos que i. $\Rightarrow$ ii, que é a implicação que vamos precisar mais tarde em nosso trabalho. Notemos que para o caso $\kappa=\aleph_{0}$ temos o conhecido Teorema de Baire (que não precisa da celularidade enumerável). Mas em geral a condição de celularidade enumerável é necessária, como pode-se ver no exemplo exibido depois do teorema:

Teorema 0.13. Suponhamos $\mathbf{M A}_{\kappa}$, para $\kappa$ cardinal infinito. Se $X \neq \phi$ é um espaço topológico compacto, de Hausdorff, com celularidade enumerável e $\left\{U_{\alpha}: \alpha<\kappa\right\}$ é uma familia não vazia de conjuntos densos abertos em $X$, então

$$
\bigcap\left\{U_{\alpha}: \alpha<\kappa\right\} \neq \phi
$$


Dem: Seja $P=\{p \subseteq X: p$ é aberto e $p \neq \phi\}$, com a pré-ordem $p \preceq q$ se e só se $p \subseteq q$. Então $P$ satisfaz a c.c.c. pois $X$ tem celularidade enumerável. Para cada $\alpha<\kappa$, seja

$$
D_{\alpha}=\left\{p \in P: \bar{p} \subseteq U_{\alpha}\right\}
$$

e seja $\mathcal{D}=\left\{D_{\alpha}: \alpha<\kappa\right\}$. Vejamos que cada $D_{\alpha}$ é denso em $P$. De fato, seja $q \in P$ qualquer. Como $U_{\alpha}$ é denso no espaço $X$, temos que $U_{\alpha} \cap q \neq \phi$. Seja $x \in U_{\alpha} \cap q$. Como $X$ é regular (por ser compacto e $T_{2}$ ) e $U_{\alpha} \cap q$ é vizinhança aberta de $x$ em $X$, temos que existe $p \in P$ tal que $x \in p \subseteq \bar{p} \subseteq U_{\alpha} \cap q$. Logo $p \preceq q$ e $p \in D_{\alpha}$. Portanto, $D_{\alpha}$ é denso em $P$. $\mathbf{M A}_{\kappa}$ implica que existe $G$, filtro $\mathcal{D}$-genérico em $P$. Afirmamos que $G$ tem a propriedade da intersecção finita. Se $g_{0}, g_{1}, \ldots, g_{i} \in G$, então, da definição 0.10, existe $r \in G$ tal que $r \preceq g_{k}$ para cada $0 \leq k \leq i$ assim $\bigcap\left\{g_{k}: 0 \leq k \leq i\right\} \supseteq r \neq \phi$, o que mostra que $G$ tem a propriedade da intersecção finita. Logo da compacidade de $X$ segue que

$$
\bigcap\{\bar{p}: p \in G\} \neq \phi
$$

Por esse fato, se nós mostrarmos que $\bigcap\{\bar{p}: p \in G\} \subseteq \bigcap\left\{U_{\alpha}: \alpha<\kappa\right\}$, mostramos o teorema. Se $x \in \bigcap\{\bar{p}: p \in G\}$, então $x \in \bar{p}$ para cada $p \in G$. Logo, da Definição 0.10, como $D_{\alpha} \cap G \neq \phi$ para todo $\alpha<\kappa$, existe $q_{\alpha} \in D_{\alpha} \cap G$. Portanto $x \in \overline{q_{\alpha}} \subseteq U_{\alpha}$ para cada $\alpha<\kappa$, o que implica que $\bigcap\{\bar{p}: p \in G\} \subseteq \bigcap\left\{U_{\alpha}: \alpha<\kappa\right\}$.

Corolário 0.14. Suponhamos $\mathbf{M A}_{\kappa}$ para $\kappa$ cardinal infinito. Se $X \neq \phi$ é um espaço compacto, de Hausdorff que tem celularidade enumerável, então $X$ não é reunião de uma família $\left\{\overline{V_{\alpha}}: \alpha<\kappa\right\}$, onde $V_{\alpha}$ é um conjunto raro para cada $\alpha<\kappa$.

Exemplo 0.2. Consideremos o espaço topológico produto, $X=\left(\omega_{1}+1\right)^{\omega}$, onde $\omega_{1}+1$ tem a topologia da ordem. Sabemos que X não tem celularidade enumerável. Queremos mostrar que é reunião de $\omega_{1}$ conjuntos raros. De fato, para cada $\alpha<\omega_{1}$ definamos

$$
A_{\alpha}=\left\{f \in X:(\forall n<\omega)\left(f(n) \neq \omega_{1} \rightarrow f(n) \leq \alpha\right)\right\}
$$


- $X=\bigcup\left\{A_{\alpha}: \alpha<\omega_{1}\right\}$ :

Se $f \in X$, definamos $A^{*}=\left\{n<\omega: f(n) \neq \omega_{1}\right\}$. Logo $\left\{\left\{f(n): n \in A^{*}\right\}\right.$ é um ordinal enumerável. Então existe um ordinal $\alpha<\omega_{1}$ tal que $\bigcup\left\{f(n): n \in A^{*}\right\}<$ $\alpha<\omega_{1}$. Assim, se $n<\omega$ tal que $f(n) \neq \omega_{1}$, então $f(n) \leq \alpha$. Portanto $f \in A_{\alpha}$.

- Para cada $\alpha<\omega_{1}, A_{\alpha}$ é fechado em $X$ :

Seja $f \notin A_{\alpha}$, então existe $n<\omega$ tal que $f(n) \neq \omega_{1}$ e $f(n)>\alpha$. Consideremos

$$
V=\left(\omega_{1}+1\right) \times \cdots \times\left(\omega_{1}+1\right) \quad \times \underbrace{\alpha, \omega_{1}}\left[\times \quad\left(\omega_{1}+1\right) \times\left(\omega_{1}+1\right) \times \cdots\right.
$$

fator de indice $n$

Logo $f \in V \subseteq X \backslash A_{\alpha}$, o que mostra que $A_{\alpha}$ é fechado em $X$.

- $\operatorname{int}_{X}\left(A_{\alpha}\right)=\phi$, para cada $\alpha<\omega_{1}$ :

Suponhamos que $\operatorname{int}_{X}\left(A_{\alpha}\right) \neq \phi$, então podemos tomar $f \in \operatorname{int}_{X}\left(A_{\alpha}\right)$ e consideremos uma vizinhança aberta de $f, W=\bigcap_{i \in I_{0}} \Pi_{i}^{-1}\left[V_{i}\right]$ tal que $I_{0}$ seja um subconjunto finito de $\omega, V_{i}$ seja um aberto de $\omega_{1}+1$ para cada $i \in I_{0}$ e $W \subseteq \operatorname{int}_{X}\left(A_{\alpha}\right)$. Agora, consideremos ordinais $\beta$ e $n_{0}$ tais que $\alpha<\beta<\omega_{1}$ e $i<n_{0}$ para cada $i \in I_{0}$. Definamos uma função $g: \omega \longrightarrow \omega_{1}+1$ do seguinte modo:

$g(m)=\left\{\begin{array}{l}f(m), \text { se } m \neq n_{0} \\ \beta \text { se } m=n_{0}\end{array}\right.$

Temos claramente que $g \in W \subseteq A_{\alpha}$, mas $g\left(n_{0}\right) \neq \omega_{1}$ e $g\left(n_{0}\right)=\beta>\alpha$, o que é absurdo. Portanto, $\operatorname{int}_{X}\left(A_{\alpha}\right)=\phi$.

Concluímos que $X=\bigcup\left\{A_{\alpha}: \alpha<\omega_{1}\right\}$, onde para cada $\alpha<\omega_{1}, A_{\alpha}$ é um conjunto raro.

$0.27\left(\alpha\right.$-escala em $\left.\omega^{\omega}\right)$. Seja $\alpha$ um ordinal. $S \subseteq \omega^{\omega}$ é dita uma $\alpha$-escala em $\omega^{\omega}$ se $S=\left\{x_{\beta}: \beta<\alpha\right\}$ e S safistaz as seguintes duas condições:

i. $x_{\theta}<_{*} x_{\beta}$, para $\theta<\beta<\alpha e$

ii. para cada $p \in \omega^{\omega}$, existe $\beta<\alpha$ tal que $p<_{*} x_{\beta}$. 
Onde a pré-ordem $<_{*}$ é a definida em 0.5.

Teorema 0.15 (MA). Seja $H \subseteq \omega^{\omega}$ tal que $|H|<2^{\aleph_{0}}$. Então existe $f \in \omega^{\omega}$ tal que para cada $h \in H, h<_{*} f .($ Portanto, $\mathfrak{b}=\mathfrak{d}=\mathfrak{c})$.

Dem: Consideremos o conjunto

$$
P=\left\{(s, E): s \in \bigcup_{n<\omega} \omega^{n}, E \subseteq H \text { finito }\right\}
$$

com a seguinte pré-ordem:

$(s, E) \preceq\left(s^{\prime}, E^{\prime}\right) \Longleftrightarrow s^{\prime} \subseteq s, E^{\prime} \subseteq E \mathrm{e}\left(\forall k \in \operatorname{Dom}(s) \backslash \operatorname{Dom}\left(s^{\prime}\right)\right)\left(\forall h \in E^{\prime}\right)(h(k)<s(k))$.

Dois pares $(s, E)$ e $\left(s, E^{\prime}\right)$ são sempre compatíveis, pois existe $\left(s, E \cup E^{\prime}\right) \in P$ tal que $\left(s, E \cup E^{\prime}\right) \preceq(s, E)$ e $\left(s, E \cup E^{\prime}\right) \preceq\left(s, E^{\prime}\right)$. Assim, se $M \subseteq P$ é uma família não enumerável, dado que $\bigcup_{n<\omega} \omega^{n}$ é enumerável, existe $s \in \bigcup_{n<\omega} \omega^{n}$ tal que $(s, E)$ e $\left(s, E^{\prime}\right)$ pertencem a $M$, para convenientes subconjuntos finitos $E, E^{\prime}$ contidos em $H$. Logo eles são compatíveis e portanto $M$ não pode ser anticadeia, o que mostra que $P$ satisfaz c.c.c.

Agora, para cada $h \in H$, definimos

$$
D_{h}=\{(s, E) \in P: h \in E\}
$$

e para cada $n<\omega$,

$$
F_{n}=\{(s, E) \in P: n \in \operatorname{Dom}(s)\}
$$

Afirmamos que $D_{h}$ e $F_{n}$ são densos em $P$, respectivamente, para cada $h \in H$ e para cada $n<\omega$. De fato, seja $(s, E) \in P$ então existe $(s, E \cup\{h\}) \in P$ tal que $(s, E \cup\{h\}) \preceq(s, E)$. Então $D_{h}$ é denso em $P$ para cada $h \in H$.

Por outro lado, seja $n<\omega$. Se $n \in \operatorname{Dom}(s)$, então $(s, E) \preceq(s, E)$ e $(s, E) \in F_{n}$; se $n \notin \operatorname{Dom}(s)$, então tomamos $s^{\prime} \in \omega^{n+1}$ definido da seguinte forma:

$$
s^{\prime}(k)=\left\{\begin{array}{l}
s(k), \quad \text { se } k \in \operatorname{Dom}(s) \\
\max \{h(k)+1: h \in E\}, \text { caso contrário }
\end{array}\right.
$$


e claramente $\left(s^{\prime}, E\right) \in F_{n}$ e $\left(s^{\prime}, E\right) \preceq(s, E)$, o que mostra que $F_{n}$ é denso em $P$ para $n<\omega$.

Se tomamos $\mathcal{D}=\left\{D_{h}: h \in H\right\} \cup\left\{F_{n}: n<\omega\right\}$, então pelo Axioma de Martin, existe $G \subseteq P$ filtro $\mathcal{D}$-genérico em $P$. Seja:

$$
f=\bigcup\{s:(s, E) \in G \text { para algum } E \subseteq H \text { finito }\}
$$

Assim, $f: \omega \longrightarrow \omega$ é uma função bem definida. De fato, se $x \in \omega$ e $f_{1}, f_{2} \in f$ são tais que $x \in \operatorname{Dom}\left(f_{1}\right) \cap \operatorname{Dom}\left(f_{2}\right)$, então por ser $G$ um filtro $\mathcal{D}$-genérico em $P$, existe $\left(f_{3}, H_{3}\right)$ em $G$, tal que $f_{1} \subseteq f_{3}$ e $f_{2} \subseteq f_{3}$, o que implica que $f_{1}(x)=f_{3}(x)=f_{2}(x)$. Portanto $f$ está bem definida. Além disso, como $G \cap F_{n} \neq \phi$ para cada $n$, temos que $\operatorname{dom}(f)=\omega$.

Vamos mostrar que para cada $h \in H$, temos que $h<_{*} f$.

Seja $h \in H$. Então, como $G \cap D_{h} \neq \phi$, podemos considerar $(s, E) \in G \cap D_{h}$, isto é, $h \in E$. Agora, se $k \in \omega \backslash \operatorname{Dom}(s)$, então como $F_{k} \cap G \neq \phi$, existe $\left(s_{1}, E_{1}\right) \in F_{k} \cap G(k \in$ $\left.\operatorname{Dom}\left(s_{1}\right)\right)$. Consideremos $\left(s_{2}, E_{2}\right) \in G$ tal que $\left(s_{2}, E_{2}\right) \preceq(s, E)$ e $\left(s_{2}, E_{2}\right) \preceq\left(s_{1}, E_{1}\right)$. Então temos que $\left(s_{2}, E_{2}\right) \in G$ é tal que $\left(s_{2}, E_{2}\right) \preceq(s, E)$ e $k \in \operatorname{Dom}\left(s_{2}\right) \backslash \operatorname{Dom}(s)$. Portanto, $f(k)=s_{2}(k)>h(k)$. Logo, $h<_{*} f$.

Corolário 0.16 (MA). Existe uma c-escala em $\omega^{\omega}$.

Dem: Seja $\omega^{\omega}=\left\{h_{\alpha}: \alpha<\mathfrak{c}\right\}$. Vamos construir uma c-escala $S=\left\{f_{\alpha}: \alpha<\mathfrak{c}\right\}$ utilizando recursão transfinita. Suponhamos definidos $\left\{f_{\alpha} \in \omega^{\omega}: \alpha<\beta\right\}$, para um ordinal $\beta$, tal que $\beta<\mathfrak{c}$. Logo tomando $H=\left\{f_{\alpha} \in \omega^{\omega}: \alpha<\beta\right\} \cup\left\{h_{\beta}\right\}$, pelo Teorema 0.15 , existe $f_{\beta} \in \omega^{\omega}$ tal que $h<_{*} f_{\beta}$, para cada $h \in H$, o que termina a demonstração. 


\section{Capítulo 1}

\section{Caracterização de $\mathbb{R} \backslash \mathbb{Q}$ e de $\omega^{\omega}$}

Neste capítulo queremos estudar o subespaço topológico dos números irracionais da reta real $\mathbb{R}$. (Denotaremos esse subespaço por $\mathbb{R} \backslash \mathbb{Q}$ ). Destacaremos algumas das suas propriedades e veremos que condições são necessárias e suficientes para que um espaço topológico seja homeomorfo a $\mathbb{R} \backslash \mathbb{Q}$. (Tal resultado será apresentado no Corolário 1.7). Em particular, vamos mostrar que $\mathbb{R} \backslash \mathbb{Q}$ é homeomorfo ao espaço $\omega^{\omega}$ com a topologia produto, onde a topologia em $\omega$ é a discreta.

Também vamos trabalhar com o espaço de Cantor $\{0,1\}^{\omega}$, que é um subespaço importante de $\omega^{\omega}$.

O primeiro resultado que vamos mostrar é que quaisquer dois densos enumeráveis em $\mathbb{R} \backslash \mathbb{Q}$ são homeomorfos e este homeomorfismo é a restrição de um homeomorfismo de $\mathbb{R} \backslash \mathbb{Q}$ a $\mathbb{R} \backslash \mathbb{Q}$. Mas antes vejamos alguns resultados que nos servirão para mostrá-lo.

Teorema 1.1 (Brower 1913, implicitamente Fréchet 1910). Sejam X e $Z$ dois conjuntos densos e enumeráveis em $\mathbb{R}$, então existe um homeomorfismo $f: \mathbb{R} \longrightarrow \mathbb{R}$, tal que $f[X]=Z$.

Dem: Suponhamos que

$$
X=\left\{x_{0}, x_{1}, \ldots\right\} \text { e } Z=\left\{z_{0}, z_{1}, \ldots\right\}
$$


e definamos indutivamente uma função $f: X \longrightarrow \mathbb{R}$ do seguinte modo:

$f\left(x_{0}\right)=z_{0} \mathrm{e}$

$f\left(x_{i}\right)$, para $i \in\{1,2, \ldots\}$, como sendo um elemento de $Z$ com o menor índice possível tal que as condições $x_{j}<x_{k}$ e $f\left(x_{j}\right)<f\left(x_{k}\right)$ sejam equivalentes para todo $j, k \leq i$.

Agora, se $w \in \mathbb{R} \backslash X$ então tomemos $\left(w_{n}\right)_{n \in \mathbb{N}} \subseteq X$ uma sequência estritamente crescente tal que $w_{n} \rightarrow w$. (Notemos que $\left(w_{n}\right)_{n \in \mathbb{N}}$ é uma sequência estritamente crescente e limitada em $X, \operatorname{assim}\left(f\left(w_{n}\right)\right)_{n \in \mathbb{N}}$ é crescente e limitada em $\mathbb{R}$ portanto convergente). Definimos $f(w)=\lim _{n \rightarrow \infty} f\left(w_{n}\right)$, se $w \in \mathbb{R} \backslash Z$. Logo podemos considerar a extensão $f: \mathbb{R} \longrightarrow \mathbb{R}$ definida como acima.

$f$ está bem definida, pois se $w \in \mathbb{R} \backslash X$ e $\left(w_{n}\right)_{n \in \mathbb{N}}$ e $\left(\overline{w_{n}}\right)_{n \in \mathbb{N}}$ são duas sequências estritamente crescentes em $X$, convergentes para $w$, vamos ter $\left(f\left(w_{n}\right)\right)_{n \in \mathbb{N}}$ e $\left(f\left(\overline{w_{n}}\right)\right)_{n \in \mathbb{N}}$ são sequências convergentes para o mesmo limite. De fato, suponha, sem perda de generalidade, que $\lim _{n \rightarrow \infty} f\left(w_{i}\right)<\lim _{n \rightarrow \infty} f\left(\overline{w_{i}}\right)$, então existe $x \in X$ tal que $\lim _{n \rightarrow \infty} f\left(w_{i}\right)<$ $f(x)<\lim _{n \rightarrow \infty} f\left(\overline{w_{i}}\right)$. Logo para cada $i \in \mathbb{N}$ temos que $w_{i}<x$ e que existe $r \in \mathbb{N}$ tal que $x<\overline{w_{r}}<w$ (pois $f(x)<\lim _{n \rightarrow \infty} f\left(\overline{w_{i}}\right)$ ). Portanto $w \leq x<w$ que é absurdo e assim $f(w)$ admite um só valor em $\mathbb{R}$.

Notemos que $f$ é estritamente crescente. Sejam $w, y \in \mathbb{R}$ com $w<y$, logo existem $x, x^{*} \in X$ tais que $w<x<x^{*}<y$. Então $f(w) \leq f(x)<f\left(x^{*}\right) \leq f(y)$. Portanto $f$ é estritamente crescente, logo injetora.

Além disso $f$ é sobrejetora, basta mostrar para $\mathbb{R} \backslash Z \subseteq i m(f)$. Tomemos $z \in \mathbb{R} \backslash Z$ e consideremos $\left(z_{i}\right)_{i \in \mathbb{N}}$ uma sequência estritamente crescente em $Z$ tal que $\lim _{i \rightarrow \infty} z_{i}=z$. Então $\left(f^{-1}\left(z_{i}\right)\right)_{i \in \mathbb{N}}$ é uma sequência estritamente crescente limitada em $\mathbb{R}$ e portanto convergente. Logo se nós tomamos $x=\lim _{i \rightarrow \infty} f^{-1}\left(z_{i}\right)$ temos que $f(x)=z$.

Para mostrar que $f$ é homeomorfismo, notemos que para $] a, b[$ aberto em $\mathbb{R}$, com $a \leq b$ e ambos em $Z$, ocorre que $\left.f^{-1}[] a, b[]=\right] f^{-1}(a), f^{-1}(b)\left[, \operatorname{logo} f^{-1}[] a, b[]\right.$ é aberto em $\mathbb{R}$. De modo similar para $] c, d[$ aberto em $\mathbb{R}$, com $c \leq d$ e ambos em $X$, ocorre que $f[] c, d[]=] f(c), f(d)[, \log f[] c, d[]$ é aberto em $\mathbb{R}$. Assim temos que $f$ é uma aplicação contínua e aberta. Logo concluímos que $f$ é homeomorfismo. 
Teorema 1.2. Se $X_{1}, X_{2}, Y_{1}$ e $Y_{2}$ são conjuntos densos e enumeráveis em $\mathbb{R}$ com $X_{1} \cap X_{2}=\phi=Y_{1} \cap Y_{2}$, então existe $f: \mathbb{R} \longrightarrow \mathbb{R}$ homeomorfismo tal que $f\left[X_{i}\right]=Y_{i}$ para $i=1,2$.

Dem: Consideremos as seguintes enumerações para os conjuntos $X_{1}, X_{2}, Y_{1}$ e $Y_{2}$ :

$$
\begin{array}{lr}
X_{1}=\left\{a_{0}, a_{1}, \ldots, a_{s}, \ldots\right\} & X_{2}=\left\{b_{0}, b_{1}, \ldots, b_{s}, \ldots\right\} \\
Y_{1}=\left\{c_{o}, c_{1}, \ldots, c_{s}, \ldots\right\} & Y_{2}=\left\{d_{0}, d_{1}, \ldots, d_{s}, \ldots\right\}
\end{array}
$$

Supondo ainda que $a_{0}<b_{0}$ e $c_{0}<d_{0}$ definamos uma função $f$ do seguinte modo: $f\left(a_{0}\right)=c_{0}$ e $f\left(b_{0}\right)=d_{0}$. Depois tomemos $f\left(a_{1}\right)$ em $Y_{1}$ como o $c_{j}$ de menor índice tal que a função

$$
f:\left\{a_{0}, b_{0}, a_{1}\right\} \longrightarrow\left\{c_{0}, d_{0}, f\left(a_{1}\right)\right\}
$$

seja estritamente crescente.

Tomemos $f\left(b_{1}\right)$ em $Y_{2}$ como sendo o $d_{j}$ de menor índice tal que a função

$$
f:\left\{a_{0}, b_{0}, a_{1}, b_{1}\right\} \longrightarrow\left\{c_{0}, d_{0}, f\left(a_{1}\right), f\left(b_{1}\right)\right\}
$$

seja estritamente crescente.

Tomemos $f\left(a_{2}\right)$ em $Y_{1}$ como sendo o $c_{j}$ de menor índice tal que a função

$$
f:\left\{a_{0}, b_{0}, a_{1}, b_{1}, a_{2}\right\} \longrightarrow\left\{c_{0}, d_{0}, f\left(a_{1}\right), f\left(b_{1}\right), f\left(a_{2}\right)\right\}
$$

seja estritamente crescente.

Depois tomemos $f\left(b_{2}\right)$ em $Y_{2}$ como sendo o $d_{j}$ de menor índice tal que a função

$$
f:\left\{a_{0}, b_{0}, a_{1}, b_{1}, a_{2}, b_{2}\right\} \longrightarrow\left\{c_{0}, d_{0}, f\left(a_{1}\right), f\left(b_{1}\right), f\left(a_{2}\right), f\left(b_{2}\right)\right\}
$$

seja estritamente crescente.

Continuando assim recursivamente, para cada $m \in \mathbb{N}$ com $m>0$, suponhamos escolhidos $f\left(a_{1}\right), f\left(a_{2}\right), \ldots, f\left(a_{m-1}\right)$ em $Y_{1}$ e $f\left(b_{1}\right), f\left(b_{2}\right), \ldots, f\left(b_{m-1}\right)$ em $Y_{2}$ tais que: 
1. O elemento $f\left(a_{m-1}\right)$ seja o $c_{j}$ de menor índice tal que $f:\left\{a_{0}, b_{0}, \ldots, a_{m-2}, b_{m-2}, a_{m-1}\right\} \longrightarrow\left\{c_{0}, d_{0}, f\left(a_{1}\right), f\left(b_{1}\right), \ldots, f\left(a_{m-2}\right), f\left(b_{m-2}\right), f\left(a_{m-1}\right)\right\}$ seja uma função estritamente crescente.

2. O elemento $f\left(b_{m-1}\right)$ seja o $d_{j}$ de menor índice tal que $f:\left\{a_{0}, b_{0}, \ldots, a_{m-1}, b_{m-1}\right\} \longrightarrow\left\{c_{0}, d_{0}, f\left(a_{1}\right), f\left(b_{1}\right), \ldots, f\left(a_{m-1}\right), f\left(b_{m-1}\right)\right\}$ seja uma funcão estritamente crescente.

Então escolhemos:

1. $f\left(a_{m}\right)$ em $Y_{1}$ como sendo o $c_{j}$ de menor índice tal que a função $f:\left\{a_{0}, b_{0}, \ldots, a_{m-1}, b_{m-1}, a_{m}\right\} \longrightarrow\left\{c_{0}, d_{0}, f\left(a_{1}\right), f\left(b_{1}\right), \ldots, f\left(a_{m-1}\right), f\left(b_{m-1}\right), f\left(a_{m}\right)\right\}$ seja estritamente crescente.

2. Logo escolhemos $f\left(b_{m}\right)$ em $Y_{2}$ como sendo o $d_{j}$ de menor índice tal que a função $f:\left\{a_{0}, b_{0}, \ldots, a_{m}, b_{m}\right\} \longrightarrow\left\{c_{0}, d_{0}, f\left(a_{1}\right), f\left(b_{1}\right), \ldots, f\left(a_{m}\right), f\left(b_{m}\right)\right\}$ seja estritamente crescente.

Nestas condições notemos que a função $f:\left(X_{1} \cup X_{2}\right) \longrightarrow\left(Y_{1} \cup Y_{2}\right)$ é uma bijeção estritamente crescente e que $f\left[X_{i}\right]=Y_{i}$ para $i=1,2$. Estendamos a função $f$ a $\mathbb{R}$ como segue: se $w \in \mathbb{R} \backslash\left(X_{1} \cup X_{2}\right)$, então tomamos uma sequência $\left(w_{n}\right)_{n \in \mathbb{N}} \subseteq X_{1} \cup X_{2}$ estritamente crescente tal que $w_{n} \rightarrow w . \quad\left(f\left(w_{n}\right)\right)_{n \in \mathbb{N}}$ é uma sequência estritamente crescente e limitada em $\mathbb{R}$, portanto ela converge. Definimos $f(w)=\lim _{n \rightarrow \infty} f\left(w_{n}\right)$. De modo análogo ao teorema anterior, mostra-se que $f$ fica bem definida e que ela é um homeomorfismo.

Assim temos um homeomorfismo $f: \mathbb{R} \longrightarrow \mathbb{R}$ onde $f\left[X_{1}\right]=Y_{1}$ e $f\left[X_{2}\right]=Y_{2}$, o que termina a prova.

O teorema que acabamos de provar, permite mostrar que quaisquer dois conjuntos densos enumeráveis em $\mathbb{R} \backslash \mathbb{Q}$ são homeomorfos e este homeomorfismo pode ser escolhido como segue: 
Teorema 1.3. Sejam $A$ e $B$ dois conjuntos densos e enumeráveis em $\mathbb{R} \backslash \mathbb{Q}$. Então existe um homeomorfismo $f: \mathbb{R} \backslash \mathbb{Q} \longrightarrow \mathbb{R} \backslash \mathbb{Q}$, tal que $f[A]=B$.

Dem: Sejam $A$ e $B$ como no teorema. Logo os conjuntos $A, B$ e $\mathbb{Q}$ são densos e enumeráveis em $\mathbb{R}$. Então pelo teorema anterior temos que existe um homeomorfismo $g: \mathbb{R} \rightarrow \mathbb{R}$ tal que $g[A]=B$ e $g[\mathbb{Q}]=\mathbb{Q}$. Agora se definimos $f=g \Upsilon_{\mathbb{R} \backslash \mathbb{Q}}: \mathbb{R} \backslash \mathbb{Q} \longrightarrow \mathbb{R} \backslash \mathbb{Q}$ temos um homeomorfismo tal que $f[A]=B$.

Agora vamos estudar espaços homeomorfos ao subespaço $\mathbb{R} \backslash \mathbb{Q}$. O teorema seguinte vai servir para encontrar condições necessárias e suficientes para saber quando um espaço topológico é homeomorfo a este subespaço. Vejamos:

Teorema 1.4. Sejam $(X, d)$ e $\left(Y, d^{\prime}\right)$ dois espaços métricos completos com $X \neq \phi \neq Y$ tais que:

1. $\left(X, \tau_{d}\right)$ e $\left(Y, \tau_{d^{\prime}}\right)$ são 0-dimensionais e

2. $\left(X, \tau_{d}\right)$ e $\left(Y, \tau_{d^{\prime}}\right)$ são de Lindelöf (ou equivalentemente que têm bases enumeráveis de abertos) e não contém compactos com interior não vazio.

Então $\left(X, \tau_{d}\right)$ e $\left(Y, \tau_{d^{\prime}}\right)$ são homeomorfos.

Dem: Para fazer a demonstração precisamos do seguinte lema:

Lema 1.5. Nas condições do Teorema 1.4, se $\phi \neq Z \subseteq X$ é aberto-fechado em $X$, então para cada $\varepsilon>0$ existe $\left\{U_{i}: i \in \mathbb{N}\right\}$, recobrimento aberto-fechado infinito de $Z$, tal que $U_{i} \cap U_{j}=\phi$ se $i \neq j$ e $\operatorname{diam}\left(U_{i}\right) \leq \varepsilon \forall i \in \mathbb{N}$.

Demostração do lema: Para cada $x \in Z$ consideremos $S_{\delta_{x}}(x)$, $\left(\operatorname{com} \delta_{x}<\varepsilon / 2\right)$ tal que $\left\{S_{\delta_{x}}(x) \cap Z: x \in Z\right\}$ seja um recobrimento aberto de $Z$ que não tem subrecobrimento finito (podemos pois $Z$ não é compacto já que não tem interior vazio). Como $X$ é 0-dimensional, para cada $x \in Z$ existe $U_{x}$ aberto-fechado tal que $x \in U_{x} \subseteq$ $S_{\delta_{x}}(x) \cap Z$. Logo $\left\{U_{x}: x \in Z\right\}$ é um recobrimento aberto-fechado de $Z$. Como $X$ 
é de Lindelöf e $Z$ é fechado (portanto de Lindelöf) existem $x_{0}, x_{1}, \ldots, x_{n}, \ldots \in Z$ tais que $Z=\bigcup\left\{U_{x_{i}}: i \in \mathbb{N}\right\}$. Assim $\left\{U_{x_{i}}: i \in \mathbb{N}\right\}$ é um recobrimento aberto-fechado enumerável de $Z$, infinito.

Agora definimos, o conjunto $\left\{U_{i}: i \in \mathbb{N}\right\}$ do seguinte modo:

$$
\begin{aligned}
U_{0} & =U_{x_{0}} \\
U_{1} & =U_{x_{1}} \backslash U_{x_{0}} \\
U_{2} & =U_{x_{2}} \backslash \bigsqcup_{i=0}^{1} U_{x_{i}} \\
& \vdots \\
U_{k} & =U_{x_{k}} \backslash \bigsqcup_{i=0}^{k-1} U_{x_{i}} \\
& \vdots
\end{aligned}
$$

Desta construção temos os seguintes fatos:

1. $\left\{U_{i}: i \in \mathbb{N}\right\}$ é um recobrimento aberto-fechado de $Z$ pois $\left\{U_{x_{i}}: i \in \mathbb{N}\right\}$ é um recobrimento aberto-fechado de $Z$.

2. $U_{i} \cap U_{j}=\phi$ se $i \neq j$

3. $\left\{U_{i}: i \in \mathbb{N}\right\}$ é um recobrimento infinito e $U_{i} \neq \phi \forall i \in \mathbb{N}$. Podemos fazer isso tirando se for necessário os $U_{i}=\phi$ e ainda assim o recobrimento vai ser infinito, já que pela nossa construção a cobertura não tem sub-recobrimento finito.

4. $U_{i} \subseteq U_{x_{i}} \subseteq S_{\delta_{x_{i}}}\left(x_{i}\right) \forall i \in \mathbb{N}$, então $\operatorname{diam}\left(U_{i}\right) \leq \varepsilon$, pois $\delta_{x_{i}}<\varepsilon$.

Vejamos que $\left(X, \tau_{d}\right)$ e $\left(Y, \tau_{d^{\prime}}\right)$ são homeomorfos.

Aplicando o Lema anterior para $Z=X$ (pois $X$ é aberto-fechado em $X$ ) e $\varepsilon=1$ temos que existe $\varphi_{1}:=\left\{U_{0}, U_{1}, \ldots, U_{n}, \ldots\right\}$ recobrimento aberto-fechado infinito de $X$, cujos elementos são não vazios e dois a dois disjuntos $\operatorname{com} \operatorname{diam}\left(U_{i}\right) \leq 1$ para cada $i \in \mathbb{N}$.

Indutivamente, para cada $k>1$ e cada $s \in \mathbb{N}^{\{1,2, \ldots, k\}}$ tomamos conjuntos $U_{s(1), s(2), \ldots, s(k)}$ abertos-fechados infinitos de $X$, dois a dois disjuntos com $\operatorname{diam}\left(U_{s(1), s(2), \ldots, s(k)}\right) \leq 1 / k$ 
tais que:

$$
U_{s(1), s(2), \ldots, s(k)}=\bigsqcup_{i=1}^{\infty} U_{s(1), s(2), \ldots, s(k), i}
$$

Notemos que para cada $x \in X$, existe um único $s_{1} \in \mathbb{N}$ tal que $x \in U_{s_{1}}$. Fixado $s_{1}$ também existe um único $s_{2} \in \mathbb{N}$ tal que $x \in U_{s_{1}, s_{2}}$, continuando assim, encontramos uma única sequência:

$$
\begin{aligned}
& s: \mathbb{N}^{+} \longrightarrow \mathbb{N} \\
& i \longmapsto \\
& s_{i}
\end{aligned}
$$

tal que $x \in U_{s(1), \ldots, s(q)} \forall q \in \mathbb{N}^{+}$. Além disso, $\{x\}=\bigcap_{q=1}^{\infty} U_{s(1), \ldots, s(q)}$. De fato, claramente $\{x\} \subseteq \bigcap_{q=1}^{n} U_{s(1), \ldots, s(q)} \forall n \in \mathbb{N}^{+}$, então $\{x\} \subseteq \bigcap_{q=1}^{\infty} U_{s(1), \ldots, s(q)}$. Se existirem $w, z$ em $\bigcap_{q=1}^{\infty} U_{s(1), \ldots, s(q)} \operatorname{com} w \neq z$, então existirá $l \in \mathbb{N}^{+}$tal que $0<1 / l<d(w, z)$. Mas, $w, z \in U_{s(1), \ldots, s(l)}$ e $\operatorname{diam}\left(U_{s(1), \ldots, s(l)}\right) \leq 1 / l \operatorname{logo} d(w, z) \leq 1 / l<d(w, z)$ o que é absurdo, portanto $w=z$. Concluímos que $\bigcap_{q=1}^{\infty} U_{s(1), \ldots, s(q)}$ não pode ter mais de um elemento. Então $\{x\}=\bigcap_{q=1}^{\infty} U_{s(1), \ldots, s(q)}$.

Podemos afirmar que para cada $s \in \mathbb{N}^{\mathbb{N}^{+}}$, existe um único $x \in X$ tal que $\{x\}=$ $\bigcap_{q=1}^{\infty} U_{s(1), \ldots, s(q)}$. De fato,

1. Claramente se existir $x$ ele é único.

2. Para cada $n \in \mathbb{N}^{+}$tomemos $x_{n} \in U_{s(1), \ldots, s(n)}$. Temos assim uma sequência $\left(x_{n}\right)_{n \in \mathbb{N}^{+}}$que é de Cauchy. Com efeito, dado $\varepsilon>0$ existe $n_{0} \in \mathbb{N}^{+}$tal que $0<1 / n_{0}<\varepsilon$; se $n \geq m \geq n_{0}$, então $x_{n}, x_{m} \in U_{s(1), \ldots, s(m)} \subseteq U_{s(1), \ldots, s\left(n_{0}\right)}$ o que implica $d\left(x_{n}, x_{m}\right) \leq 1 / n_{0}<\varepsilon$ e assim $\left(x_{n}\right)_{n}$ será de Cauchy.

Como $\mathrm{X}$ é completo, sabemos que existe $x \in X$ tal que $x_{n} \rightarrow x$ o que garante que $\{x\}=\bigcap_{q=1}^{\infty} U_{s(1), \ldots, s(q)}$, pois tomando $r \in \mathbb{N}$ temos que $\forall n \in \mathbb{N}^{+}$com $n \geq r$ $x_{n} \in U_{s(1), \ldots, s(r)}$ (que é fechado) $\operatorname{logo} x \in U_{s(1), \ldots, s(r)}$.

Procedemos agora de forma análoga no espaço $Y$ para determinar os conjuntos $V_{s(1), s(2), \ldots, s(k)}$, (como os conjuntos $U_{s(1), s(2), \ldots, s(k)}$ em $\left.X\right)$. É claro que valem resultados semelhantes aos acima, ligeiramente modificados para o espaço $Y$. 
Definamos agora uma função $f$ do seguinte modo:

$$
\begin{aligned}
& f: X \longrightarrow Y \\
& x \longmapsto f(x) \\
& \text { onde } \quad\{x\}=\bigcap_{i=1}^{\infty} U_{s(1), \ldots, s(i)} \Longleftrightarrow\{f(x)\}=\bigcap_{i=1}^{\infty} V_{s(1), \ldots, s(i)}
\end{aligned}
$$

A função $f$ está bem definida como consequência das afirmações anteriores. Essa função $f$ vai ser o homeomorfismo entre os espaços $X$ e $Y$. Vejamos que ela é de fato um homeomorfismo:

$f$ é injetora: É imediato.

$f$ é sobrejetora: Tomemos $y \in Y$, então existe uma única $s \in \mathbb{N}^{\mathbb{N}^{+}}$tal que $\{y\}=$ $\bigcap_{i=1}^{\infty} V_{s(1), \ldots, s(i)}$, assim se $\{x\}=\bigcap_{i=1}^{\infty} U_{y_{1}, \ldots, y_{i}}$ temos que $f(x)=y$.

Continuidade da $f$ : Tomemos $W \subseteq Y$ aberto e mostremos que $f^{-1}[W]$ é aberto em $X$. Seja $z \in f^{-1}[W]$ qualquer, então existe $w \in W$ tal que $f(z)=w$, onde $\{w\}=\bigcap_{i=1}^{\infty} V_{w_{1}, \ldots, w_{i}}$. Agora, como $W$ é aberto em $Y$ existe $\varepsilon>0$ tal que $S_{\varepsilon}(w) \subseteq W$. Tomemos $l \in \mathbb{N}^{+}$suficientemente grande tal que $V_{w_{1}, \ldots, w_{l}} \subseteq S_{\varepsilon}(w)$. Afirmamos que $U_{w_{1}, \ldots, w_{l}} \subseteq f^{-1}[W]$. De fato, se $t \in U_{w_{1}, \ldots, w_{l}}$ então $f(t) \in V_{w_{1}, \ldots, w_{l}} \subseteq W \operatorname{logo} t \in f^{-1}[W]$. Assim mostramos que $z \in U_{w_{1}, \ldots, w_{l}} \subseteq f^{-1}[W]$. Portanto, $f^{-1}[W]$ é aberto.

Concluímos que a função $f$ é contínua.

$f$ é aberta: Tomemos $Z \subseteq X$ aberto e mostremos que $f[Z]$ é aberto em $Y$. Seja $w \in$ $f[Z] \log$ existe $w \in Z$ tal que $w=f(z)$; suponhamos que $\{z\}=\bigcap_{i=1}^{\infty} U_{z_{1}, \ldots, z_{i}}$. Como $Z$ é aberto em $X$ existe $\varepsilon>0$ tal que $S_{\varepsilon}(z) \subseteq Z$. Tomemos $l \in N^{+}$suficientemente grande tal que $V_{z_{1}, \ldots, z_{l}} \subseteq S_{\varepsilon(z)}$. Afirmamos que $V_{z_{1}, \ldots, z_{l}} \subseteq f[Z]$, de fato, se $t \in V_{z_{1}, \ldots, z_{l}}$ então $f^{-1}(t) \in U_{z_{1}, \ldots, z_{l}} \subseteq S_{\varepsilon}(z) \subseteq Z \operatorname{logo} t \in f[z]$. Assim $w \in V_{z_{1}, \ldots, z_{l}} \subseteq f[Z]$. Portanto 
$f[Z]$ é aberto.

Concluímos que $f$ é uma aplicação aberta.

Isto mostra que $f: X \longrightarrow Y$ é um homeomorfismo de espaços topológicos.

Como dissemos anteriormente, deste teorema destacaremos os seguintes corolários importantes:

Corolário 1.6 (Baire). O subespaço $\mathbb{R} \backslash \mathbb{Q}$ da reta real e o espaço topológico $\omega^{\omega}$ com a topologia produto ( $\omega$ com a topologia discreta) são homeomorfos.

Corolário 1.7 (Alexandroff e Urysohn). Todo espaço não vazio completamente metrizável, separável e 0-dimensional que não contém subconjuntos compactos abertos não vazios é homeomorfo a $\mathbb{R} \backslash \mathbb{Q}$.

Dem: Seja $X$ um espaço com as condições do corolário, então é claro que $X$ é completamente metrizável, 0-dimensional e de Lindelöf. Só falta ver que ele não tem compactos com interior não vazio para que, pelo Teorema 1.4, X seja homeomorfo ao espaço $\mathbb{R} \backslash \mathbb{Q}$. Mas essa condição (nas condições do teorema) é equivalente a não ter compactos abertos não vazios. Com efeito, se vale a primeira condição, tomemos $F$ compacto aberto em $X$, então $F=\operatorname{int}(F) \neq \phi$. Reciprocamente, se vale a segunda condição, tomemos $F$ compacto em $X$, se $\operatorname{int}(F) \neq \phi$, então existe $B$ aberto-fechado em $X$ não vazio tal que $B \subseteq F, \operatorname{logo} B$ é um compacto aberto em $X$ não vazio, o que é absurdo. Assim o corolário está demonstrado.

Corolário 1.8. Se X é um espaço topológico de Lindelöf, completamente metrizável e 0-dimensional, então $X$ é homeomorfo a um subespaço de $\mathbb{R} \backslash \mathbb{Q}$.

Dem: Só aplicar o Teorema 1.4 para os espaços $(\mathbb{R} \backslash \mathbb{Q}) \times X$ (que tomado assim não contém compactos com interior não vazio) e $\mathbb{R} \backslash \mathbb{Q}$. 
No último corolário temos um resultado que nos permite dizer quando um espaço metrizável é homeomorfo a um subespaço de $\mathbb{R} \backslash \mathbb{Q}$, mas esse corolário tem condições que não são necessárias para que exista tal homeomorfismo, como por exemplo a condição dele ser completo. Daí o interesse no seguinte teorema:

Teorema 1.9. Se $X$ é um espaço metrizável, separável tal que $|X|<\mathfrak{c}$, então $X$ é homeomorfo a um subespaço de $\mathbb{R} \backslash \mathbb{Q}$.

Dem: Vejamos que $X$ admite uma base de abertos-fechados em $X$. Tomemos $x \in X$ e $U$ aberto qualquer em $X$ contendo o ponto $x$. Como $X$ é $T_{3 \frac{1}{2}}$ temos que existe uma função $f: X \longrightarrow[0,1]$ contínua tal que $f(x)=0$ e $f(y)=1$ para cada $y \notin U$. Como $\mid\left[0, \frac{1}{2}\left[\mid=\mathfrak{c}\right.\right.$ e $\mid f^{-1}\left[\left[0, \frac{1}{2}[] \mid<\mathfrak{c}\right.\right.$ temos que existe $y \in\left[0, \frac{1}{2}[\right.$ tal que $y$ não pertence à imagem de $f$. Logo existe $W_{U, x}=f^{-1}[[0, y]]=f^{-1}\left[\left[0, y[]\right.\right.$ (ou seja $W_{U, x}$ é aberto-fechado em $X$ ) tal que $x \in W_{U, x} \subseteq U$. Portanto o conjunto $\left\{W_{U, x}: U\right.$ é aberto em $X$ e $\left.x \in X\right\}$ é uma base de abertos-fechados em $X$.

Consideremos $B=\left\{B_{i}: i \in \mathbb{N}\right\}$ uma base de abertos-fechados de $X$. Para cada $i \in \mathbb{N}$ definamos:

$$
\begin{aligned}
f_{i}: X & \longrightarrow\{0,1\} \\
x & \longmapsto f_{i}(x)= \begin{cases}0 & \text { se } x \in B_{i} \\
1 & \text { se } x \notin B_{i}\end{cases}
\end{aligned}
$$

Assim definamos a seguinte função:

$$
\begin{aligned}
\Phi: X & \longrightarrow\{0,1\}^{\omega} \\
x & \longmapsto \Phi(x)=\left(f_{i}(x)\right)_{i \in \mathbb{N}}
\end{aligned}
$$

Como $\{0,1\}^{\omega}$ é subespaço de $\omega^{\omega}$, se nós mostrarmos que $\Phi$ é um homeomorfismo sobre a sua imagem, acabaremos a demonstração do teorema. 
Injetividade da $\Phi$ : Sejam $x$ e $y$ elementos diferentes de $X$. Logo como $X$ é $T_{1}$, existe $l \in \mathbb{N}$ tal que $x \in B_{l}$ e $y \notin B_{l}$. Assim $\Phi(x)=\left(f_{i}(x)\right)_{i \in \mathbb{N}} \neq\left(f_{i}(y)\right)_{i \in \mathbb{N}}=\Phi(y)$ (pois $f_{l}(x)=0$ e $\left.f_{l}(y)=1\right)$. Portanto $\Phi$ é injetora.

$\Phi$ é contínua: Seja $\bigcap\left\{\prod_{i}^{-1}\left[V_{i}\right]: i=1,2, \ldots, n\right\}$ um aberto básico de $\{0,1\}^{\omega}$ com $\phi \neq V_{i} \varsubsetneqq\{0,1\}$. Queremos mostrar que $\Phi^{-1}\left[\bigcap\left\{\prod_{i}^{-1}\left[V_{i}\right]: i=1,2, \ldots, n\right\}\right]$ é aberto em $X$. De fato, seja $x \in \Phi^{-1}\left[\bigcap\left\{\prod_{i}^{-1}\left[V_{i}\right]: i=1,2, \ldots, n\right\}\right]$, então $f_{i}(x)=\left(\prod_{i} \circ \Phi\right)(x) \in V_{i}$ $\forall i=1,2, \ldots, n$. Seja $W=\bigcap_{i=1}^{n}\left\{W_{i}: W_{i}=B_{i}\right.$ se $f_{i}(x)=0$ ou $W_{i}=X \backslash B_{i}$ se $\left.f_{i}(x)=1\right\}$. Assim $W$ é um aberto em $X$ tal que $x \in W \subseteq \Phi^{-1}\left[\bigcap\left\{\prod_{i}^{-1}\left[V_{i}\right]: i=1,2, \ldots, n\right\}\right]$. Portanto $\Phi$ é contínua.

$\Phi$ é aberta sobre a sua imagem: Seja $B_{k} \in B$, queremos mostrar que $\Phi\left[B_{k}\right]$ é aberto em $\{0,1\}^{\omega} \cap \Phi[X]$. Com efeito, seja $y \in \Phi\left[B_{k}\right]$, então existe $x \in B_{k}$ tal que $\Phi(x)=y$. Tomemos $W=\prod_{k}^{-1}[\{0\}]$, aberto em $\{0,1\}^{\omega}$, assim $y \in W \cap \Phi[X] \subseteq \Phi\left[B_{k}\right]$, provando-se que $\Phi$ é aberta sobre a sua imagem.

Dos fatos antes mencionados temos que $\Phi$ é um homeomorfismo sobre a sua imagem e assim o teorema é demonstrado.

Vimos que um espaço topológico satisfazendo certas condições pode ser visto como um subespaço de $\mathbb{R} \backslash \mathbb{Q}$. Uma pergunta natural que surge depois deste resultado é: quais são as condições necessárias para um espaço topológico ser um subespaço denso em $\mathbb{R} \backslash \mathbb{Q}$ ? Vejamos alguns lemas que ajudarão a dar uma resposta para esse problema:

Lema 1.10. Seja $Y$ um subespaço denso de um espaço Hausdorff X. Se um subconjunto compacto de $Y$ é aberto em $Y$, então ele também é aberto em $X$.

Dem: Seja $A$ um compacto aberto em $Y$, e seja $B$ um aberto em $X$ tal que $A=B \cap Y$. queremos mostrar que $B=A$. 
Claramente, $A \subseteq B$. Por outro lado, se $b \in B$ temos que para cada $U \subseteq X$, vizinhança aberta de $b$, temos que $B \cap U$ é também uma vizinhança aberta de $b$ em $X$. Então $U \cap A=B \cap U \cap Y \neq \phi$; ou seja $b \in c l_{X}(A)=A$ (pois $A$ é compacto em $X$ ), $\log \mathrm{B} \subseteq A$.

Lema 1.11. Sejam Y um espaço metrizável, 0-dimensional e separável, que não contém subconjuntos compactos abertos não vazios e X um espaço completamente metrizável contendo $Y$. Então existe um conjunto $W$, homeomorfo a $\mathbb{R} \backslash \mathbb{Q}$, tal que:

$$
Y \subseteq W \subseteq \operatorname{cl}(Y) \subseteq X
$$

Dem: Sem perda de generalidade, como $c l(Y)$ é completamente métrizável, podemos supor $Y$ denso em $X$. Assim $X$ é também separável.

Seja $M$ um conjunto enumerável tal que $M \subseteq X \backslash Y \subseteq \operatorname{cl}(M)$ e tomemos

$$
X_{1}=X \backslash M
$$

De $X_{1}$ podemos dizer que:

1. É $G_{\delta}$, pois $X_{1}=\bigcap\{X \backslash\{m\}: m \in M\}$

2. É denso em $X$, pois $Y \subseteq X_{1}$

3. Não contém subconjuntos compactos abertos não vazios. De fato, se existir $B \subseteq$ $X_{1}$ compacto, aberto e não vazio, então como $\operatorname{cl}\left(X_{1}\right)=X$, pelo Lema 1.10 temos que $B$ é aberto em $X$. Mas pelas hipóteses sobre $Y$ no lema, temos que $B \nsubseteq Y$ $\operatorname{logo} B \cap(X \backslash Y) \neq \phi$ portanto $B \cap M \neq \phi$, que é absurdo.

Assim, (substituindo $X$ por $X_{1}$, se for necessário) vemos que $X$ não contém compactos abertos não vazios, então, pelo Teorema de Tumarkin (Teorema 0.12), existe $W, G_{\delta}$ contido em $X$, tal que $Y \subseteq W \subseteq X$ e $\operatorname{Ind} Y=\operatorname{Ind} W$ (então, como $\operatorname{Ind} Y=0$, 
pelo Teorema $0.6 W$ é 0-dimensional).

Como $W$ é completamente metrizável (pois é $G_{\delta}$ ), separável, 0-dimensional e (por um raciocínio análogo a à demonstração para $X_{1}$ em 3.) $W$ não contém compactos abertos não vazios, temos que $W$ é homeomorfo a $\mathbb{R} \backslash \mathbb{Q}$.

Dos lemas apresentados anteriormente, podemos deduzir o seguinte teorema que da uma resposta ao problema proposto anteriormente.

Teorema 1.12. Todo espaço metrizável, separável, 0-dimensional, que não contém compactos abertos não vazios pode ser densamente imerso em $\mathbb{R} \backslash \mathbb{Q}$.

Dem: Basta tomar $X$ como completamento de $Y$ no Lema 1.11.

Em resultados anteriores vimos algumas condições que um espaço topológico deve ter para ser homeomorfo a um subespaço de $\mathbb{R} \backslash \mathbb{Q}$. Agora, nós daremos mais alguns resultados onde damos condições para que um espaço topológico contenha subespaços homeomorfos a $\mathbb{R} \backslash \mathbb{Q}$ ou homeomorfos a subespaços (como por exemplo $\{0,1\}^{\omega}$ ) de $\mathbb{R} \backslash \mathbb{Q}$.

Teorema 1.13. Todo espaço completamente metrizável e denso em si mesmo, contém o espaço de Cantor.

Dem: Seja $(X, \tau)$ um espaço topológico com as condições do teorema e seja $\rho$ uma métrica tal que $(X, \rho)$ seja completo e a topologia de $(X, \rho)$ seja a topologia $\tau$. Para cada sequência $\left(i_{0}, i_{1}, \ldots, i_{k}\right)$ de 0 's e 1 's, definamos indutivamente conjuntos não vazios e abertos $V_{i_{0}, i_{1}, \ldots, i_{k}} \subseteq X$ com diâmetro menor que $1 /(k+1)$ tais que:

1. $\bar{V}_{i_{0}, i_{1}, \ldots, i_{k}, 0} \cap \bar{V}_{i_{0}, i_{1}, \ldots, i_{k}, 1}=\phi$.

2. $\bar{V}_{i_{0}, i_{1}, \ldots, i_{k}, i} \subseteq V_{i_{0}, i_{1}, \ldots, i_{k}}$ para cada $i=0,1$.

Definamos

$$
\begin{aligned}
\varphi:\{0,1\}^{\omega} & \longrightarrow X \\
s & \longmapsto \varphi(s)
\end{aligned}
$$


onde $\{\varphi(s)\}=\bigcap\left\{V_{s(0), s(1), \ldots, s(k)}: k \in \mathbb{N}\right\}$.

A funcão $\varphi$ está bem definida; isso se verifica de modo análogo a como mostramos que a função $f$ no teorema 1.4 estava bem definida.

Vamos mostrar que $\varphi$ é um homeomorfismo sobre a sua imagem para mostrar o teorema.

\section{Claramente $\varphi$ é injetora.}

2. Vejamos que $\varphi$ é contínua, seja $W \subseteq X$ aberto, queremos mostrar que $\varphi^{-1}[W]$ é aberto em $\{0,1\}^{\omega}$. Se $s \in \varphi^{-1}[W]$ então $\varphi(s) \in W \operatorname{logo}$ tomemos $k \in \mathbb{N}$ suficientemente grande tal que $\varphi(s) \in V_{s(0), s(1), \ldots, s(k)} \subseteq W$ e consideremos $Z=$ $\{s(0)\} \times\{s(1)\} \times \cdots \times\{s(k)\} \times\{0,1\} \times \cdots \times\{0,1\}$ aberto em $\{0,1\}^{\omega}$. Mostremos que $s \in Z \subseteq \varphi^{-1}[W]$. Com efeito, se $z \in Z$ então $s(i)=z(i)$ para todo $o \leq i \leq k$ $\operatorname{logo} \varphi(z) \in V_{s(0), s(1), \ldots, s(k)} \subseteq W$, ou seja $z \in \varphi^{-1}[W]$. Segue-se que $\varphi^{-1}[W]$ é aberto em $\{0,1\}^{\omega}$.

3. $\varphi$ é aberta, seja $Z=\bigcap\left\{\prod_{i}^{-1}\left[W_{i}\right]: 0 \leq i \leq n\right\}$ um aberto básico de $\{0,1\}^{\omega}$, onde $W_{i} \varsubsetneqq\{0,1\}$. Queremos mostrar que $\varphi[Z]$ é aberto em $X \cap \varphi\left[\{0,1\}^{\omega}\right]$. De fato, seja $y \in \varphi[Z]$ então existe $z \in Z$ tal que $y=\varphi(z)$ onde $\{\varphi(z)\}=\bigcap\left\{V_{z(0), z(1), \ldots, z(k)}\right.$ : $k \in \mathbb{N}\}$; se nós mostrarmos que $V_{z(0), z(1), \ldots, z(n)} \cap \varphi\left[\{0,1\}^{\omega}\right] \subseteq \varphi[Z]$ mostraremos que $\varphi[Z]$ é aberto em $X \cap \varphi\left[\{0,1\}^{\omega}\right]$. Com efeito, se $w \in V_{z(0), z(1), \ldots, z(n)} \cap \varphi\left[\{0,1\}^{\omega}\right]$ então $w \in V_{z(0), z(1), \ldots, z(n)}$ e $w=\varphi(v)$ para algum $v \in\{0,1\}^{\omega}, \log 0 v(k)=z(k)$ para cada $0 \leq k \leq n$, portanto $v \in Z$.

Concluímos que o espaço topológico $X$ contém um subespaço homeomorfo ao espaço de Cantor.

Teorema 1.14. Se X é um espaço topológico completamente metrizável de Lindelöf, sem pontos isolados, então existe um subespaço $Y$ denso em $X$ tal que $Y$ é homeomorfo $a \mathbb{R} \backslash \mathbb{Q}$. 
Dem: Do teorema da metrização (Teorema 0.7) podemos considerar $\mathcal{B}$ uma base de abertos $\sigma$-discreta de $X$. Suponhamos

$$
\mathcal{B}=\bigcup\left\{\mathcal{B}_{n}: n \in \mathbb{N}\right\}
$$

onde $\mathcal{B}_{n}$ é uma família discreta de abertos para cada $n \in \mathbb{N}$. Como $X$ é de Lindelöf, podemos supor cada $\mathcal{B}_{n}$ enumerável. Assim definimos para cada $n \in \mathbb{N}$, conjuntos

$$
A_{n}=\bigcup\left\{F r(\Omega): \Omega \in \mathcal{B}_{n}\right\}
$$

Temos que cada $A_{n}$ :

1. É fechado, pois $\left\{\operatorname{Fr}(\Omega): \Omega \in \mathcal{B}_{n}\right\}$ é uma família localmente finita e sabemos que união de uma família de fechados localmente finita é fechada.

2. Tem interior vazio, pois para cada $\Omega \in \mathcal{B}_{n}, \operatorname{Fr}(\Omega)$ é fechado com interior vazio em $X \operatorname{logo} \bigcup\left\{F r(\Omega): \Omega \in \mathcal{B}_{n}\right\}$ tem interior vazio.

Seja $D$ um denso enumerável em $X$ e consideremos

$$
Y=X \backslash\left(\bigcup\left\{A_{n}: n \in \mathbb{N}\right\} \cup D\right)
$$

Notemos que $Y$ é denso em $X$, pois dado que $X$ é um espaço de Baire, o conjunto $Y=X \backslash\left(\bigcup\left\{A_{n}: n \in \mathbb{N}\right\}\right)$ é denso em $X$. Agora, como todos os abertos não vazios de $X$ tem cardinalidade não enumerável e $D$ é enumerável, para cada $U \subseteq X$ aberto $B \cap Y \neq \phi$. Portanto $Y$ é denso em $X$.

Vamos mostrar que $Y$ é homeomorfo a $\mathbb{R} \backslash \mathbb{Q}$ :

\section{1. $Y$ é completamente metrizável;}

como $Y=X \backslash\left(\bigcup\left\{A_{n}: n \in \mathbb{N}\right\} \cup D\right)=\bigcap\left\{X \backslash A_{n}: n \in \mathbb{N}\right\} \cap \bigcap\{X \backslash\{d\}: d \in D\}$ temos que $Y$ é um $G_{\delta}$, portanto completamente metrizável.

2. $Y$ é de Lindelöf, pois $X$ é hereditariamente separável. 
3. $Y$ é 0-dimensional, pois $B \uparrow_{Y}=\{V \cap Y: V \in B\}$ é uma base de abertos-fechados de $Y$.

4. $Y$ não tem compactos abertos não vazios. De fato, suponha $F$ compacto e aberto em $Y$ vamos mostrar que $F=\phi$. Se $F \neq \phi$, então tomemos $W \subseteq X$ aberto não vazio, tal que $F=W \cap Y$. Vejamos que $F=W$, claramente $F \subseteq W$; por outro lado, se $w \in W$, e tomando $U \subseteq X$ vizinhança qualquer aberta de $w$, ocorre que $W \cap U$ é também vizinhança aberta de $w$ em $X$, mas como $Y$ é denso em $X, F \cap U=W \cap U \cap Y \neq \phi$ então $w \in c_{Y}(F)=F$.

Assim existe $d \in D$ tal que $d \in F$, que é absurdo. Portanto $F=\phi$.

Destes fatos, pelo corolário 1.7 , concluímos que $Y$ é homeomorfo a $\mathbb{R} \backslash \mathbb{Q}$.

Até agora neste capítulo vimos algumas propriedades que caracterizam o espaço topológico $\mathbb{R} \backslash \mathbb{Q}$. Mas nós também trabalhamos com o espaço de Cantor $\{0,1\}^{\omega}$ em alguns dos resultados dados; a seguir vamos estudar um pouco esse espaço.

Teorema 1.15. Todo espaço topológico X metrizável e compacto é imagem contínua do espaço de Cantor.

Dem: Seja $\rho$ uma métrica em $X$ compatível com a topologia do espaço. Existem $n_{0} \geq 1\left(s_{0}=2^{n_{0}}\right)$ e uma família de fechados (compactos) não vazios $\left\{F_{i}: i \in\{0,1\}^{n_{0}}\right\}$ tais que $\operatorname{diam}\left(F_{i}\right)<1$ para todo $i \in\{0,1\}^{n_{0}} \mathrm{e}$

$$
X=\bigcup\left\{F_{i}: i \in\{0,1\}^{n_{0}}\right\}
$$

(Os $F_{i}$ 's podem ser repetidos.)

Agora, existe $n_{1} \geq 1$ tal que cada compacto $F_{i}$ anterior, com $i \in\{0,1\}^{n_{0}}$, se escreve como união de $2^{n_{1}}$ fechados não vazios com diâmetro menor que $1 / 2$; ou seja

$$
F_{i}=\bigcup\left\{F_{i, j}: j \in\{0,1\}^{\left\{n_{0}, n_{0}+1, \ldots, n_{0}+n_{1}-1\right\}}\right\}
$$


onde cada $F_{i, j}$ é um fechados não vazio em $X$, com diâmetro menor que $\frac{1}{2}$ para cada $j \in\{0,1\}\left\{n_{0}, n_{0}+1, \ldots, n_{0}+n_{1}-1\right\}$.

Concluímos que

$$
X=\bigcup\left\{F_{k}: k \in\{0,1\}^{n_{0}+n_{1}}\right\}
$$

onde $F_{k}$ é um compacto não vazio com $\operatorname{diam}\left(F_{j}\right)<\frac{1}{2}$ para cada $k \in\{0,1\}^{n_{0}+n_{1}}$. Continuando assim por indução, para cada $m \in \mathbb{N} \backslash\{0\}$, representamos $X$ como união de $s_{m}=2^{n_{0}+\cdots+n_{m}}$ conjuntos não vazios $F_{i_{0}, \ldots, i_{m}}$ compactos em $X$, com diâmetro menor que $\frac{1}{2^{m}}$, tais que $F_{i_{0}, \ldots, i_{m-1}}=\bigcup\left\{F_{i_{0}, \ldots, i_{m-1}, j}: j \in\{0,1\}^{\left\{N(m), N(m)+1, \ldots, N(m)+n_{m}-1\right\}}\right\}$, onde $N(m)=n_{0}+n_{1}+\cdots+n_{m-1}$ para todo $m>0$; ou seja $X=\bigcup\left\{F_{j}: j \in\{0,1\}^{n_{0}+n_{1}+\cdots+n_{m}}\right\}$ onde $F_{j}$ é compacto não vazio em $X \operatorname{com} \operatorname{diam}\left(F_{j}\right)<\frac{1}{2^{m}}$ para cada $j \in\{0,1\}^{n_{0}+n_{1}+\cdots+n_{m}}$.

Denotemos por $N_{k}=\{0,1, \ldots, N(k+1)-1\}$ para cada $k \in \mathbb{N}$ e definamos:

$$
\begin{aligned}
\psi:\{0,1\}^{\omega} & \longrightarrow X \\
j & \longmapsto \psi(j)
\end{aligned}
$$

onde $\{\psi(j)\}=\bigcap\left\{F_{j\left\lceil_{N_{k}}\right.}: k \in \mathbb{N}\right\}$.

Observe que $\psi$ está bem definida pois a sequência de compactos não vazios $F_{\left.j\right|_{N_{0}}} \supseteq$ $F_{\left.j\right|_{N_{1}}} \supseteq \cdots \supseteq F_{j \uparrow_{N_{k}}} \supseteq \cdots$ dentro do compacto $X$ tem intersecção não vazia e pela escolha dos diâmetros temos que a intersecção é unitária.

Claramente $\psi$ é uma aplicação sobrejetora.

Vejamos que $\psi$ é contínua. Seja $\varepsilon>0$ e consideremos $S_{\varepsilon}(x)$ onde $x \in X$ é um ponto qualquer. Mostremos que $\psi^{-1}\left[S_{\varepsilon}(x)\right]$ é aberto em $\{0,1\}^{\omega}$. Com efeito, seja $z \in \psi^{-1}\left[S_{\varepsilon}(x)\right]$, então $\psi(z) \in S_{\varepsilon}(x)$ onde $\{\psi(z)\}=\bigcap\left\{F_{z \uparrow_{N_{k}}}: k \in \mathbb{N}\right\}$. Tomemos $m \in \mathbb{N}$ suficientemente grande tal que $F_{\left.z\right|_{N_{m}}} \subseteq S_{\varepsilon}(x)$. Logo se consideramos $V=$ $\{z(0)\} \times\{z(1)\} \times \cdots \times\left\{z\left(n_{0}+\cdots+n_{m}-1\right)\right\} \times\{0,1\} \times \cdots \times\{0,1\}$, então claramente $z \in V \subseteq \psi^{-1}\left[S_{\varepsilon}(x)\right]$. Portanto $\psi$ é contínua. 
O próximo teorema nos vai dar uma caracterização do espaço de Cantor:

Teorema 1.16 (Alexandroff). Um espaço metrizável não vazio, compacto, 0-dimensional, sem pontos isolados é homeomorfo ao espaço de Cantor.

Dem: Suponhamos que na demonstração do teorema anterior tomarmos o conjunto

$$
\left\{F_{j}: j \in\{0,1\}^{n_{0}+n_{1}+\cdots+n_{k}}, k \in \mathbb{N}\right\}
$$

de tal modo que, para cada $k \in \mathbb{N}$, se tomamos $i, j \in\{0,1\}^{n_{0}+n_{1}+\cdots+n_{k}}$ diferentes, então $F_{i} \cap F_{j}=\phi$. Então logo para cada ponto $y \in X$ existiria uma única sequência $s \in\{0,1\}^{\omega}$ tal que $F_{\left.s\right|_{N_{0}}} \supseteq F_{\left.s\right|_{N_{1}}} \supseteq \cdots \supseteq F_{s \uparrow_{N_{k}}} \supseteq \cdots$ seja uma sequência de compactos em $X$ contendo $y$ e viceversa; para cada sequência $s \in\{0,1\}^{\omega}$ vai existir um único ponto $\psi(s) \in X$. Deste modo a aplicação $\psi$ é injetora e portanto um homeomorfismo (pois $\psi$ pelo teorema anterior é contínua, sobrejetora entre espaços compactos).

Observação: Se $X$ é um espaço topológico com as condições do Teorema 1.16, então para todo compacto aberto-fechado não vazio $A$ de $X$, existem compactos $A_{1}$ e $A_{2}$ abertos-fechados em $X$ não vazios e disjuntos tais que $A=A_{1} \cup A_{2}$.

De fato, Seja $\rho$ uma métrica compatível com a topologia de $X$ e tomemos $x \in A$, $\operatorname{logo}$ como $X$ não contém pontos isolados, existe $\varepsilon>0$ tal que $S_{\varepsilon}(x) \varsubsetneqq A$, mas como $X$ é 0-dimensional, existe $A_{1} \subseteq X$ aberto-fechado tal que $x \in A_{1} \subseteq S_{\varepsilon}(x)$. Temos portanto que $A=A_{1} \cup\left(A \backslash A_{1}\right)$ onde $A_{1}$ e $A \backslash A_{1}$ são dois conjuntos com as condições que queríamos.

Da observação acima, pelo fato de $X$ ser um espaço metrizável, compacto, 0dimensional sem pontos isolados, para cada $\varepsilon>0$ podemos escrever $X$ como união de compactos (abertos-fechados) não vazios, dois a dois disjuntos e de diâmetro menor que $\varepsilon$, de tal modo que o número de elementos de tal união (sem repetições) seja uma potência de 2 .

Isto termina a demonstração. 
Deste teorema, junto com os Teoremas 1.12 e 1.1, podemos obter uma caracterização do subespaço dos números racionais.

Corolário 1.17. Todo espaço não vazio metrizável, enumerável, denso em si mesmo é homeomorfo ao espaço dos números racionais.

Dem: Seja $X$ um espaço nas condições do corolário. Então:

1. $X$ é um espaço 0-dimensional. De fato, sejam $x \in X$ e $U \subseteq X$ vizinhança aberta de $x$. Como $X$ é $T_{3 \frac{1}{2}}$, existe $f_{U, x}: X \longrightarrow[0,1]$ contínua, tal que $f_{U, x}(x)=0$ e $f_{U, x}(y)=1$ para cada $y \in X \backslash U$. Agora, dado que $U$ é enumerável, então existe $c \in[0,1]$ tal que $c \notin i m\left(f_{U, x}\right)$. Tomando $W_{(U, x)}=f_{U, x}^{-1}[[0, c]]=f_{U, x}^{-1}[[0, c[]$, temos um aberto-fechado em $X$ tal que $x \in W_{(U, x)} \subseteq U$. Portanto o conjunto $\left\{W_{(U, x)}: U\right.$ é aberto em $X$ contendo o ponto $x$ de $\left.X\right\}$ é uma base de abertosfechados de $X$.

2. $X$ não tem compactos abertos não vazios; pois se existir $U \subseteq X$ compacto aberto não vazio, então $U$ seria um espaço metrizável, compacto, 0-dimensional não vazio sem pontos isolados, logo pelo Teorema 1.16, temos que $U$ é homeomorfo ao espaço $\{0,1\}^{\omega}$ então $|U|=2^{\omega}$ que é absurdo.

\section{3. $X$ é metrizável e separável}

Logo temos $X$ nas condições do Teorema 1.12 e portanto existe $W \subseteq \mathbb{R} \backslash \mathbb{Q}$ denso tal que $X$ é homeomorfo a $W$. Logo $W$ é denso e enumerável em $\mathbb{R}$ e pelo Teorema 1.1 ocorre que $W$ é homeomorfo a $\mathbb{Q}$.

Na introdução da dissertação, se mencionou que L. B. Lawrence em [10, prop. 1] diz o seguinte:

"Se $X$ e $Y$ são espaços topológicos e $Y$ é completamente metrizável, separável e não $\sigma$-compacto, então $X \times Y$ é normal se e só se $X \times(\mathbb{R} \backslash \mathbb{Q})$ é normal". 
Logo é interessante ver que $\mathbb{R} \backslash \mathbb{Q}$ não é $\sigma$-compacto. O Teorema 1.18 abaixo envolve um pequeno cardinal e justamente mostra isso.

Vamos introduzir um cardinal topológico invariante e calcular o seu valor para $\mathbb{R} \backslash \mathbb{Q}$. Consideremos a função cardinal "compact covering number" $k c$ dada por

$$
\begin{gathered}
k c(X)=\text { cardinalidade mínima de uma cobertura do espaço topológico } X \\
\text { por subconjuntos compactos de } X .
\end{gathered}
$$

Uma pergunta natural que podemos fazer é qual é o valor para $k c(\mathbb{R} \backslash \mathbb{Q})$. Observemos que $\{\{x\}: x \in \mathbb{R} \backslash \mathbb{Q}\}$ é uma cobertura formada por c compactos de $\mathbb{R} \backslash \mathbb{Q}$, portanto $k c(\mathbb{R} \backslash \mathbb{Q}) \leq \mathfrak{c}$. Por outro lado, $\mathbb{R} \backslash \mathbb{Q}$ é um espaço de Baire e como sabemos que todo compacto de $\mathbb{R} \backslash \mathbb{Q}$ é fechado com interior vazio (destacado por Hechler, ver [8]), temos que se $\mathbb{R} \backslash \mathbb{Q}$ fosse união enumerável de compactos, então ele teria interior vazio, o que é absurdo. Assim $\omega<k c(\mathbb{R} \backslash \mathbb{Q})$. Portanto

$$
\omega<k c(\mathbb{R} \backslash \mathbb{Q}) \leq \mathfrak{c}
$$

O seguinte teorema dá uma resposta mais precisa para esse problema:

Teorema 1.18. $k c(\mathbb{R} \backslash \mathbb{Q})=\mathfrak{d}$

Dem: Lembremos que:

$\mathfrak{d}=\min \left\{|D|: D \subseteq \omega^{\omega}\right.$ é uma família dominante em $\left.\left(\omega^{\omega}, \leq_{*}\right)\right\}$

$\mathfrak{d}_{1}=\min \left\{|D|: D \subseteq \omega^{\omega}\right.$ é uma família cofinal em $\left.\left(\omega^{\omega}, \leq\right)\right\}$

onde, para $f, g \in \omega^{\omega}, f \leq g$ quer dizer que para cada $n \in \mathbb{N}, f(n) \leq g(n)$ e $f \leq_{*} g$ quere dizer que o conjunto $\{n \in \mathbb{N}: g(n)<f(n)\}$ é finito.

Nós usaremos para mostrar o teorema o fato de que $\mathbb{R} \backslash \mathbb{Q}$ é homeomorfo a $\omega^{\omega}$ e que $\mathfrak{d}=\mathfrak{d}_{1}$. Assim é suficiente mostrar que $k c\left(\omega^{\omega}\right)=\mathfrak{d}_{1}$.

Vejamos que $\mathfrak{d}_{1} \leq k c\left(\omega^{\omega}\right)$ : Seja $\mathcal{V}$ um recobrimento por compactos de $\omega^{\omega}$ tal que $|\mathcal{V}|=k c\left(\omega^{\omega}\right)$. Para cada $K \in \mathcal{V}$ e $n<\omega$ temos que $\prod_{n}[K]$ (projeção sobre a $n$-ésima 
coordenada) é compacto em $\omega$, logo é finito. Assim podemos definir para cada $K \in \mathcal{V}$ uma função $f_{K} \in{ }^{\omega} \omega$ dada por $f_{K}(n)=\max \left(\prod_{n}[K]\right)$ para cada $n<\omega$. O conjunto $\left\{f_{K}: K \in \mathcal{V}\right\}$ é cofinal em $\left(\omega^{\omega}, \leq\right)$. Portanto temos que $\mathfrak{d}_{1} \leq k c\left(\omega^{\omega}\right)$.

Vejamos que $k c\left(\omega^{\omega}\right) \leq \mathfrak{d}_{1}$ : Seja $D \subset \omega^{\omega}$ cofinal em $\left(\omega^{\omega}, \leq\right)$ tal que $|D|=\mathfrak{d}_{1}$. Consideremos o conjunto $\left\{K_{f}: f \in D\right\}$, onde $K_{f}=\prod_{i<\omega}[0, f(i)]([0, f(i)]$ é o intervalo em $\mathbb{N}$ ). Claramente $K_{f}$ é compacto em $\omega^{\omega}$, mostremos agora que esse conjunto é de fato um recobrimento de $\omega^{\omega}$. Seja $g \in \omega^{\omega}$, como $D$ é cofinal em $\left(\omega^{\omega}, \leq\right)$ existe $f \in D$ tal que $g \leq f$. Assim para cada $n \in \mathbb{N}$ temos que $0 \leq g(n) \leq f(n)$, então $g \in \prod_{i<\omega}[0, f(i)]=K_{f}$. Portanto $k c\left(\omega^{\omega}\right) \leq \mathfrak{d}_{1}$. 


\section{Capítulo 2}

\section{Não normalidade de produtos de espaços normais por $\mathbb{R} \backslash \mathbb{Q}$}

Neste capítulo veremos alguns exemplos que servem para mostrar que nem sempre o produto de um espaço normal por um espaço metrizável e separável é normal. Na verdade o que vamos estudar é um problema um pouco mais específico. Vamos querer estudar espaços topológicos com algumas condições (por exemplo ser normal ou de Lindelöf) para os quais o produto com $\mathbb{R} \backslash \mathbb{Q}$ não é normal.

Para um espaço topológico $X$ que é regular e de Lindelöf, se sabe que o produto $X \times(\mathbb{R} \backslash \mathbb{Q})$ é normal se e só se $X \times(\mathbb{R} \backslash \mathbb{Q})$ é de Lindelöf. Tal afirmação será mostrada no Teorema 3.4 (página 85), mas ao longo deste capítulo vamos assumir tal asserção como verdadeira.

Na primeira seção vamos ver um exemplo exibido por E. Michael [11]. Ele descreveu um espaço topológico normal, conhecido como a reta de Michael, tal que o produto deste espaço com $\mathbb{R} \backslash \mathbb{Q}$ não é normal.

Nas seções seguintes, veremos outros exemplos de espaços topológicos; usando por exemplo a Hipótese do Contínuo ou o Axioma de Martin (na seção 2) ou o conceito de concentração e a asserção $\mathfrak{b}=\omega_{1}$ (na seção 3), cujo produto com o subespaço dos 
números irracionais não é normal.

\subsection{Reta de Michael}

O teorema a seguir foi demonstrado em $Z F C$ sem nenhum axioma adicional por Michael [11]. Ele mostra que o produto de espaços normais por espaços metrizáveis separáveis nem sempre é normal.

Definição 2.1. A reta de Michael, denotada por $\mathbb{M}$, é o conjunto $\mathbb{R}$ com a topologia

$$
\Theta_{\mathbb{M}}=\{U \cup V: U \text { é um aberto em } \mathbb{R} e V \subseteq \mathbb{R} \backslash \mathbb{Q}\} .
$$

Teorema 2.1. Existe um espaço topológico normal tal que o seu produto com $\mathbb{R} \backslash \mathbb{Q}$, como subespaço de $\mathbb{R}$, não é normal.

Dem: Seja $Y$ o conjuntos dos irracionais como subespaço métrico de $\mathbb{R}$ e $X$ o intervalo $[0,1]$ com a topologia

$$
\Theta=\{U \cup V: U \text { é aberto usual em }[0,1], V \subseteq[0,1] \backslash \mathbb{Q}\}
$$

Observação: $O$ espaço $X$ é o subespaço $[0,1]$ de $\mathbb{M}$. Quando nos referimos a $[0,1]$ o consideraremos como subespaço de $\mathbb{R}$.

Vejamos que $X$ é hereditariamente paracompacto.

Antes de fazer a demostração notemos que se $H \subseteq X$ é um subespaço, então os abertos de $H$ são da forma $W_{1} \cup W_{2}$ onde $W_{1} \subseteq H \cap Y$ e $W_{2}$ é aberto no subespaço $H$; pois se $U$ é aberto em $H$, então $U=(W \cup V) \cap H$, onde $W \subseteq \mathbb{R} \backslash \mathbb{Q}$ e $V$ é aberto em $[0,1]$. Logo $U=(W \cap H) \cup(V \cap H)$ onde $(W \cap H) \subseteq H \cap Y$ e $(V \cap H)$ é aberto 
em $H$ (com a topologia de subespaço de $\mathbb{R}$ ). Assim para mostrar que $X$ é hereditariamente paracompacto basta mostrar que $X$ é paracompacto, pois a paracompacidade dos subespaços de $X$ mostra-se de forma análoga.

Para mostrar que $X$ é paracompacto, tomemos uma cobertura de $X$ por abertos em $\Theta$

$$
\mathcal{V}=\left\{U_{s} \cup V_{s}: s \in S\right\}
$$

onde $V_{s} \subseteq[0,1] \cap Y$ e $U_{s}$ é aberto em $[0,1]$. Logo a cobertura aberta $\left\{U_{s}: s \in S\right\}$ do espaço $U=\bigcup\left\{U_{s}: s \in S\right\} \subseteq[0,1]$, tem um refinamento localmente finito $\left\{U_{t}^{*}: t \in T\right\}$ (pois $[0,1]$ é um espaço métrico, portanto hereditariamente paracompacto). Assim o conjunto

$$
\{\{x\}: x \in X \backslash U\} \cup\left\{U_{t}^{*}: t \in T\right\}
$$

é um refinamento aberto localmente finito da cobertura $\mathcal{V}$ de $X$, portanto $X$ é paracompacto.

Como $X$ é $T_{2}$, temos um espaço topológico normal $X$ e um espaço métrico separável $Y$. Queremos mostrar que $X \times Y$ não é normal.

Sejam $A=(X \backslash Y) \times Y$ e $B=\{(y, y): y \in[0,1] \backslash \mathbb{Q}\}$, conjuntos fechados e disjuntos de $X \times Y$. Vejamos que eles não podem ser separados por abertos disjuntos em $X \times Y$.

Afirmação: $[0,1] \backslash \mathbb{Q}$ não é $F_{\sigma}$ em $X$.

De fato, suponhamos que

$$
[0,1] \backslash \mathbb{Q}=\bigcup\left\{F_{i}: i \in \mathbb{N}\right\}
$$

com $F_{i}$ fechado em $X$. $\operatorname{Logo}[0,1] \cap \mathbb{Q}=\bigcap\left\{X \backslash F_{i}: i \in \mathbb{N}\right\}$, e supondo $X \backslash F_{i}=$ $\left(Z_{i} \cap X\right) \cup V_{i}$ (onde $Z_{i}$ é aberto em $\mathbb{R}$ e $V_{i} \subseteq[0,1] \backslash \mathbb{Q}$ ) temos que $[0,1] \cap \mathbb{Q}=\bigcap\left\{Z_{i} \cap X\right.$ : $i \in \mathbb{N}\}$, ou seja $[0,1] \backslash \mathbb{Q}=\bigcup\left\{X \backslash Z_{i}: i \in \mathbb{N}\right\}$. Mas $X \backslash Z_{i}$ é um fechado com interior vazio em $[0,1] \backslash \mathbb{Q}$ para cada $i \in \mathbb{N}$, e como $[0,1] \backslash \mathbb{Q}$ é um espaço de Baire, temos que 
$[0,1] \backslash \mathbb{Q}=\bigcup\left\{X \backslash Z_{i}: i \in \mathbb{N}\right\}$ tem interior vazio em $[0,1] \backslash \mathbb{Q}$, o que é absurdo. Portanto, $[0,1] \backslash \mathbb{Q}$ não é $F_{\sigma}$ em $X$.

Seja $W$ um aberto em $X \times Y$ contendo $B$. Para cada $n \in \mathbb{N}^{+}$tomemos

$$
U_{n}=\left\{x \in[0,1] \backslash \mathbb{Q}:\left(\{x\} \times S_{1 / n}(x)\right) \subseteq W\right\}
$$

O conjunto $\left\{U_{n}: n \in \mathbb{N}^{+}\right\}$é um recobrimento de $[0,1] \backslash \mathbb{Q}$ e pela afirmação acima, como $[0,1] \backslash \mathbb{Q}$ não é $F_{\sigma}$ em $X$, então existe $k \in \mathbb{N}^{+}$tal que $\overline{U_{k}} \cap(X \backslash Y) \neq \phi$. Fixemos $x \in \overline{U_{k}} \cap(X \backslash Y)$, e tomemos $y \in Y$ tal que $|x-y|<1 / 2 k$. Assim $(x, y) \in A$ e vamos mostrar que toda vizinhança aberta de $(x, y)$ intersecta $W$. Assim teremos que $A$ e $B$ não podem ser separados por abertos disjuntos. De fato, seja $S \times T$ uma vizinhança aberta de $(x, y)$ em $X \times Y$. Tomemos $x^{\prime} \in S \cap U_{k}$ tal que $\left|x-x^{\prime}\right|<1 / 2 k$. Então $\left|x^{\prime}-y\right| \leq\left|x^{\prime}-x\right|+|x-y|<\frac{1}{2 k}+\frac{1}{2 k}=\frac{1}{k} \operatorname{logo}\left(x^{\prime}, y\right) \in\left\{x^{\prime}\right\} \times S_{1 / k}\left(x^{\prime}\right) \subseteq W$, pois $x^{\prime} \in U_{k}$. Logo $\left(x^{\prime}, y\right) \in(S \times T) \cap W$, o que finaliza a demonstração.

Observação: No teorema acima, foi mostrado que o espaço topológico $(X, \Theta)$ é normal, mas esse espaço não é de Lindelöf.

De fato, como o conjunto $X \cap \mathbb{Q}$ é enumerável, podemos supor

$$
X \cap \mathbb{Q}=\left\{q_{n}: n \in \mathbb{N}, n \geq 2\right\}
$$

Seja $\left.H_{n}=X \cap\right] q_{n}-\frac{1}{2^{n+1}}, q_{n}+\frac{1}{2^{n+1}}[$, para cada $n \in \mathbb{N}$ com $n \geq 2$. Claramente

$$
\left\{H_{n}: n \in \mathbb{N}, n \geq 2\right\} \cup\{\{x\}: x \in X \backslash \mathbb{Q}\}
$$

é um recobrimento aberto de $X$, vamos mostrar que esse recobrimento não tem subrecobrimento enumerável usando um pouco de teoria da medida. Seja $\mu$ a medida de Lebesgue em $\mathbb{R}$ (ver [7, exemplo 2.4.1]). Dado que $\mathbb{Q} \cap X \subseteq \bigcup\left\{H_{n}: n \in \mathbb{N}, n \geq 2\right\}$, $\mu(X)=\mu([0,1])=1$ e que

$$
\mu\left(\bigcup\left\{H_{n}: n \in \mathbb{N}, n \geq 2\right\}\right) \leq \sum_{n=2}^{\infty} \mu\left(H_{n}\right) \leq \sum_{n=2}^{\infty} \frac{1}{2^{n}}=\frac{1}{2}
$$

temos que o conjunto $X \backslash \bigcup\left\{H_{n}: n \in \mathbb{N}, n \geq 2\right\} \subseteq X \backslash \mathbb{Q}$ tem medida não nula, logo não é enumerável (pois todo conjunto enumerável em $\mathbb{R}$ tem medida de Lebesgue nula). 
Portanto concluímos que a cobertura de $X$ dada em (2.1.1), não tem sub-recobrimento enumerável, mostrando que $X$ não é de Lindelöf.

\section{2 $\mathrm{CH}, \mathrm{MA}$ e produto de espaços de Lindelöf com os irracionais}

O espaço $X$ tomado na seção anterior não era de Lindelöf, logo poderíamos pensar que a propriedade ser de Lindelöf para um espaço normal ajudaria para que seu produto topológico com um espaço métrico separável seja normal. Mas essa asserção é falsa como veremos no teorema seguinte.

Até agora, não é conhecido em ZFC, um exemplo de um espaço topológico normal e de Lindelöf tal que o seu produto com o $\mathbb{R} \backslash \mathbb{Q}$ não seja normal. Por isso como veremos ao longo desta seção, vamos precisar de axiomas adicionais para encontrar alguns contra-exemplos.

O seguinte teorema foi mostrado por Michael, ver [11].

Teorema 2.2. O produto de um espaço normal de Lindelöf e um espaço metrizável separável não é necessariamente normal.

Dem: Nós mostraremos que existem um espaço normal de Lindelöf $X$ e um espaço metrizável $Y$ tais que $X \times Y$ não é normal.

Construção do espaço $Y$ : Seja $C$ o conjunto dos subconjuntos compactos de $[0,1]$, com a topologia usual, que não são enumeráveis. Sabemos que $|C|=\mathfrak{c}$, então podemos indexar $C$ do modo seguinte:

$$
C=\left\{K_{\alpha}: \alpha<\mathfrak{c}\right\}
$$

Consideremos os subconjuntos $Y=\left\{y_{\alpha}: y_{\alpha} \in K_{\alpha}\right\}$ e $B=\left\{b_{\alpha}: b_{\alpha} \in K_{\alpha}\right\}$ definidos por recursão transfinita do seguinte modo: $y_{0} \neq b_{0}$ com $y_{0}, b_{0} \in K_{0}$ e para cada ordinal 
$\alpha \operatorname{com} 0<\alpha<\mathfrak{c}$ tomemos $y_{\alpha} \neq b_{\alpha}$ ambos em $K_{\alpha}$ tais que $y_{\alpha}, b_{\alpha} \notin \bigsqcup_{0<i<\alpha}\left\{y_{i}, b_{i}\right\}$ (ou seja que eles sejam diferentes de todos os $y_{i}$ 's e $b_{i}$ 's anteriores; isto é possível pois $\left|K_{\alpha}\right|=\mathfrak{c}$, para todo $\left.\alpha<\mathfrak{c}\right)$. Assim $Y \subseteq[0,1]$ é um subconjunto não enumerável que tem a propriedade de que qualquer subconjunto compacto contido nele é enumerável. De fato, seja $M \subseteq Y$ compacto e suponhamos que $M$ não é enumerável, então $M \in C$ e assim existe $\alpha<\mathfrak{c}$ tal que $M=K_{\alpha} \operatorname{logo} y_{\alpha} \in M$ e $b_{\alpha} \notin Y$, mas $b_{\alpha} \in K_{\alpha}=M$, o qual é absurdo e portanto $M$ tem que ser enumerável.

Consideremos o conjunto $Y$ construído acima como subespaço métrico de $\mathbb{R}$ e tomemos

$X=[0,1]$ com a topologia

$$
\Theta=\{W \cup V: W \subseteq Y \text { e } V \text { aberto usual em }[0,1]\}
$$

Vejamos que $(X, \Theta)$ é um espaço topológico de Lindelöf.

Seja $\left\{F_{i}: i \in T\right\}$ um recobrimento aberto de $(X, \Theta)$. Para cada $i \in T$ supomos $F_{i}=W_{i} \cup V_{i}$, onde $W_{i} \subseteq Y$ e $V_{i}$ é um aberto usual em $[0,1]$.

Seja $M=\bigsqcup_{i \in T} V_{i}$. Então $[0,1] \backslash M$ é fechado em [0,1] e portanto compacto. Agora, como $[0,1] \backslash M$ está contido em $Y$, ele tem que ser enumerável. Logo para cada $x \in$ $[0,1] \backslash M$ existe $i_{x} \in T$ tal que $x \in W_{i_{x}}$. Seja $T_{1} \subseteq T$ dado por $T_{1}=\left\{i_{x}: x \in[0,1] \backslash M\right\}$ ( $T_{1}$ é enumerável). Agora, como $[0,1]$ é hereditariamente Lindelöf (por ter base enumerável de abertos) e $M=\bigsqcup_{i \in T} V_{i}$, temos que existe $T_{2} \subseteq T$ enumerável tal que $M=\bigsqcup_{n \in T_{2}} V_{n}$. Tomemos $T_{3}=T_{1} \cup T_{2}$. Assim $T_{3}$ é enumerável e vale que $X=\bigsqcup_{i \in T_{3}} F_{i}$. Portanto $X$ é de Lindelöf.

Vejamos que o espaço $X$ é normal.

É claro que $X$ é $T_{1}$ pois $[0,1]$ na topologia usual é $T_{1}$ e $\Theta$ é um refinamento da topologia usual. Para mostrar que ele é $T_{4}$ consideremos $A, B$ fechados disjuntos em $X$, e consideremos os seguintes conjuntos: 


$$
\begin{array}{ll}
A_{1}=A \backslash Y & B_{1}=B \backslash Y \\
A_{2}=A \cap Y & B_{2}=B \cap Y
\end{array}
$$

Para cada $a \in A_{1}$ temos que $a \in X \backslash B$, então existe $\varepsilon_{a}>0$ tal que $S_{\varepsilon_{a}}(a) \subseteq X \backslash B$, onde $S_{\varepsilon_{a}}(a)$ é a bola de raio $\varepsilon_{a}$ e centro em $a$ em $[0,1]$ (note que como $a \notin Y$, podemos tomar um aberto em $\Theta$ tomando $V=S_{\varepsilon_{a}}(a)$ e $\left.W=\phi\right)$. Do mesmo modo, para cada $b \in B_{1}$ temos que $b \in X \backslash A$, então existe $\varepsilon_{b}>0$ tal que $S_{\varepsilon_{b}}(b) \subseteq X \backslash A$. Tomemos $Z_{1}=\bigsqcup_{a \in A_{1}} S_{\varepsilon_{a} / 3}(a)$ e $Z_{2}=\bigsqcup_{b \in B_{1}} S_{\varepsilon_{b} / 3}(b), . \quad$ Logo temos que $A_{1} \subseteq Z_{1}, B_{1} \subseteq Z_{2}$ e $Z_{1} \cap Z_{2}=\phi$. Para mostrar este último fato, suponhamos que existe $x \in Z_{1} \cap Z_{2}=\phi$. Então existem $a \in A_{1}$ e $b \in B_{1}$ tais que $x \in S_{\varepsilon_{a} / 3}(a) \cap S_{\varepsilon_{b} / 3}(b)$. Logo $|a-b| \leq$ $|a-x|+|x-b|<\varepsilon_{a} / 3+\varepsilon_{b} / 3 \leq \frac{2}{3} \max \left\{\varepsilon_{a}, \varepsilon_{b}\right\}$ então $b \in S_{\varepsilon_{a} / 3}(a)$ ou $a \in S_{\varepsilon_{b} / 3}(b)$ o que é absurdo.

Finalmente se tomarmos $Z_{3}=A_{2} \cup Z_{1}$ e $Z_{4}=B_{2} \cup Z_{2}$, temos que $Z_{3}, Z_{4}$ são abertos em $X$ tais que $A \subseteq Z_{3}, B \subseteq Z_{4}$, e $Z_{3} \cap Z_{4}=\phi$. Portanto, $X$ é normal.

Vejamos que $X \times Y$ não é normal.

Consideremos os conjuntos: $A=(X \backslash Y) \times Y$ e $B=\{(x, x): x \in Y\}$ contidos em $X \times Y$. Eles são disjuntos, fechados e não podem ser separados por abertos disjuntos em $X \times Y$. De fato:

Que eles são conjuntos disjuntos e fechados é claro.

Mostremos agora que $A$ e $B$ não podem ser separados por abertos disjuntos em $X \times Y:$

Primeiro vejamos que $Y$ não é $F_{\sigma}$ em $X$. Suponhamos por absurdo que $Y=\bigsqcup_{n=1}^{\infty} F_{n}$ onde $F_{n}$ é fechado em $X$. Assim para cada $n \in \mathbb{N}^{+} X \backslash F_{n}$ é aberto em $X$. Vamos supor que $X \backslash F_{n}=W_{n} \cup V_{n}$, onde $W_{n} \subseteq Y$ e $V_{n}$ é aberto em [0,1]. Então $F_{n}=\left(X \backslash W_{n}\right) \cap\left(X \backslash V_{n}\right)$ e $X \backslash V_{n}$ é compacto em $[0,1]$ (por ser fechado). Mas como $Y$ é 
não enumerável, temos que existe $r \in \mathbb{N}^{+}$tal que $F_{r}$ é não enumerável e portanto $X \backslash V_{r}$ é não enumerável. Então temos que $X \backslash V_{r}$ é um compacto não enumerável em $[0,1]$ logo pertence a $C$. Suponhamos que $X \backslash V_{r}=K_{\alpha}$. Então $y_{\alpha}, b_{\alpha} \in X \backslash V_{r} \operatorname{com} y_{\alpha} \in Y$ e $b_{\alpha} \notin Y$. Agora como $W_{r} \subseteq Y$ temos que $X \backslash Y \subseteq X \backslash W_{r}$. Logo dado que $b_{\alpha} \notin Y$ ocorre que $b_{\alpha} \in X \backslash W_{r}$, e portanto $b_{\alpha} \in\left(X \backslash W_{r}\right) \cap\left(X \backslash V_{r}\right)=F_{r}$. Então $b_{\alpha} \in Y$ o que é absurdo pois $b_{\alpha} \notin Y$. Concluímos que $Y$ não é um $F_{\sigma}$ em $\mathrm{X}$.

Seja agora $V$ um aberto em $X \times Y$ tal que $B \subseteq V$, Para cada $k \in \mathbb{N}^{+}$tomemos $U_{k}=\left\{x \in Y:\{x\} \times S_{1 / k}(x) \subseteq V\right\}$. Notemos que os $U_{k}$ 's cobrem $Y$. Mas como $Y$ não é $F_{\sigma}$ em $X$ existe $k \in \mathbb{N}^{+}$tal que $\overline{U_{k}} \cap(X \backslash Y) \neq \phi$. Tomemos $x \in \overline{U_{k}} \cap(X \backslash Y)$ e $y \in Y$ tal que $|x-y|<\frac{1}{2 k}$, então $(x, y) \in(X \backslash Y) \times Y=A$. Queremos mostrar que qualquer vizinhança aberta de $(x, y)$ intersecta $V$. De fato, suponhamos $(x, y) \in R \times S$, onde $R \times S$ é subconjunto aberto de $X \times Y$. Então como $R$ é vizinhança aberta de $x$ em $X$ e $x \in \overline{U_{k}}$, temos que $U_{k} \cap R \neq \phi$. Seja $x^{\prime} \in U_{k} \cap R$ e tal que $\left|x-x^{\prime}\right|<\frac{1}{2 k}$. Então $\left|x^{\prime}-y\right| \leq\left|x^{\prime}-x\right|+|x-y|<\frac{1}{2 k}+\frac{1}{2 k}=\frac{1}{k} . \operatorname{Logo}\left(x^{\prime}, y\right) \in\left\{x^{\prime}\right\} \times S_{1 / k}\left(x^{\prime}\right) \subseteq V$, pois $x^{\prime} \in U_{k}$, o que termina a demonstração..

Concluímos que o espaço $X \times Y$ não é normal.

Observação: No exemplo exibido na proposição acima, só usamos do espaço [0,1] o fato dele ser compacto, metrizável e não ter pontos isolados. Assim é possível generalizar esse exemplo para espaços com essas condições. O conjunto $Y$ neste caso pode ser obtido de forma similar tomando $C$ como o conjunto de todos os subconjuntos compactos de $X$ que não são enumeráveis $\left(|C|=2^{\omega}\right)$.

Anteriormente, vimos dois exemplos nos quais mostramos que o produto de um espaço normal $X$ por um espaço métrico separável $Y$ nem sempre é normal. Na verdade, no primeiro exemplo, tomamos $Y$ como sendo o subespaço dos números irra- 
cionais e $X$ um espaço que não era de Lindelöf. No segundo exemplo, o espaço $X$ que exibimos era de Lindelöf, mas $Y$ não era o subespaço dos números irracionais. No entanto, se nós assumirmos a Hipótese do Contínuo $(\mathrm{CH})$, podemos dar mais um exemplo onde vamos tomar o espaço $Y$ como sendo o subespaço dos números irracionais e $X$ como um espaço topológico que além de ser normal, também será de Lindelöf.

Até o final da demonstração do teorema abaixo nós supomos que $\omega_{1}=\mathfrak{c}$ (Hipótese do Contínuo).

Seja

$$
\Omega=\left\{\Omega^{*}: \mathbb{Q} \subseteq \Omega^{*} \text { e } \Omega^{*} \text { é aberto em } \mathbb{R}\right\}
$$

Vejamos que $|\Omega|=\omega_{1}$. De fato, dado que $\mathbb{R}$ admite base enumerável, e cada elemento de $\Omega$ é união de alguns desses elementos, então $|\Omega| \leq 2^{\omega}=\mathfrak{c}$. Por outro lado, $|\Omega|$ não é enumerável, pois senão $\mathbb{Q}=\bigcap \Omega$ e sabemos que $\mathbb{Q}$ não é un $G_{\delta} \mathrm{em} \mathbb{R}$. Temos assim que $\omega<|\Omega|\left(\omega_{1} \leq|\Omega|\right)$. Portanto $|\Omega|=\mathfrak{c}=\omega_{1}$.

Indexamos $\Omega$ por

$$
\Omega=\left\{\Omega_{\alpha}: \alpha<\omega_{1}\right\} .
$$

Definamos por recursão transfinita um conjunto $Y=\left\{y_{\alpha}: \alpha<\omega_{1}\right\}$ do seguinte modo:

1. $y_{0} \in \Omega_{0} \cap(\mathbb{R} \backslash \mathbb{Q})$

2. Para cada ordinal $\alpha$ com $0<\alpha<\omega_{1}$, supondo definidos $y_{\beta}$ para cada $\beta<\alpha$, então tomamos $y_{\alpha} \in\left(\bigcap\left\{\Omega_{\beta}: \beta \leq \alpha\right\} \backslash\left\{y_{i}: i<\alpha\right\}\right) \cap(\mathbb{R} \backslash \mathbb{Q})$. Podemos fazer isto porque $\mathbb{Q}$ não é $G_{\delta}$ em $\mathbb{R}$.

Teorema 2.3 (CH). O conjunto $X=\mathbb{Q} \cup Y$ com a topologia $\Theta=\{W \cup V: W \subseteq Y$ e Vaberto relativo de $X$ em $\mathbb{R}\}$ é um espaço regular e de Lindelöf (normal) tal que o espaço produto $X \times(\mathbb{R} \backslash \mathbb{Q})$ não é normal.

Dem: Vejamos que $(X, \Theta)$ é de Lindelöf e regular (logo, normal): 
$X$ é de Lindelöf: Seja $G=\left\{G_{i}: i \in I\right\}$ um recobrimento por abertos de $X$. Suponhamos para cada $i \in I$ que $G_{i}=W_{i} \cup\left(Z_{i} \cap X\right)$, onde $W_{i} \subseteq Y$ e $Z_{i}$ é um aberto em $\mathbb{R}$. Existe $J \subseteq I$ enumerável tal que $\mathbb{Q} \subseteq \bigcup\left\{Z_{i}: i \in J\right\}$. Tomemos $\alpha$, com $\alpha<\omega_{1}$, tal que $\Omega_{\alpha}=\bigcup\left\{Z_{i}: q \in J\right\}$. Logo se $\lambda>\alpha$, então $y_{\lambda} \in \Omega_{\alpha}$ ou seja $\left\{y_{\lambda}: \lambda>\alpha\right\} \subseteq \bigcup\left\{Z_{i}: i \in J\right\}$. Deste modo, $Y \backslash \bigcup\left\{Z_{i}: i \in J\right\} \subseteq\left\{y_{\lambda}: \lambda \leq \alpha\right\}$ que é enumerável pois $\alpha<\omega_{1}$; podemos tomar para cada $\lambda \leq \alpha, i_{\lambda} \in I$ tal que $y_{\lambda} \in G_{i_{\lambda}}$. Seja $I_{1}=J \cup\left\{i_{\lambda}: \lambda \leq \alpha\right\}$ e assim $X=\bigcup_{i \in I_{1}} G_{i}$, onde $I_{1}$ é enumerável. Portanto $X$ é de Lindelöf.

$X$ é regular: Sejam $x \in X$ e $F \subseteq X$ fechado quaisquer tais que $x \notin F$. Então $X \backslash F$ é aberto em $X$, logo podemos supor $X \backslash F=W \cup(Z \cap X)$ onde $W \subseteq Y$ e $Z$ é um aberto de $\mathbb{R}$. Agora,

$$
F=(X \backslash W) \cap(X \backslash(Z \cap X))=(X \backslash W) \cap((X \backslash Z) \cup(X \backslash X))=(X \backslash W) \cap(X \backslash Z)
$$

como $x \notin F$, então $x \notin X \backslash W$ ou $x \notin X \backslash Z$.

1. Se $x \notin X \backslash Z=(\mathbb{R} \backslash Z) \cap X$, então $x \in Z$ aberto em $\mathbb{R}$, logo existe $V_{1}^{*}$ aberto de $\mathbb{R}$ tal que $x \in V_{1}^{*} \subseteq \overline{V_{1}^{*}} \subseteq Z$. Sejam $V_{1}=V_{1}^{*} \cap X$ e $V_{2}=\left(\mathbb{R} \backslash \overline{V_{1}^{*}}\right) \cap X=X \backslash \overline{V_{1}^{*}}$. Notemos que $V_{1}, V_{2}$ são abertos de $X$ tais que $V_{1} \cap V_{2}=\phi, x \in V_{1}$ e $F \subseteq X \backslash Z \subseteq$ $X \backslash \overline{V_{1}^{*}}=V_{2}$.

2. Se $x \notin X \backslash W$, então $x \in W$. Tomemos $V_{1}=\{x\} \in \Theta$ e $V_{2}=X \backslash\{x\} \in \Theta$. Logo $x \in V_{1}, F \subseteq V_{2}$ e $V_{1} \cap V_{2}=\phi$.

Portanto $X$ é $T_{3}$ e, como é facil ver que $X$ é também $T_{1}$, concluímos que $X$ é regular.

\section{Vejamos que $X \times(\mathbb{R} \backslash \mathbb{Q})$ não é normal:}

Afirmação: $Y$ não é $F_{\sigma}$ em $X$.

De fato, se $Y=\bigcup\left\{F_{i}: i \in \mathbb{N}\right\}$ onde $F_{i}$ é fechado em $X$, então $\mathbb{Q}=X \backslash Y=$ $\bigcap\left\{X \backslash F_{i}: i \in \mathbb{N}\right\}$. Suponhamos, para cada $i \in \mathbb{N}$, que $X \backslash F_{i}=\left(Z_{i} \cap X\right) \cup W_{i}$, onde $Z_{i}$ é aberto em $\mathbb{R}$ e $W_{i} \subseteq Y$. Logo, $\mathbb{Q}=\bigcap\left\{\left(Z_{i} \cap X\right) \cup W_{i}: i \in \mathbb{N}\right\}=\bigcap\left\{\left(Z_{i} \cap X\right): i \in \mathbb{N}\right\}$. 
Mas para cada $i \in \mathbb{N}$, como $\mathbb{Q} \subseteq Z_{i}$, existe $\alpha_{i}<\omega_{1}$ tal que $Z_{i}=\Omega_{\alpha_{i}}$. Então $\mathbb{Q} \subseteq \bigcap\left\{\Omega_{\alpha_{i}} \cap X: i \in \mathbb{N}\right\}=\bigcap\left\{Z_{i} \cap X: i \in \mathbb{N}\right\}=\mathbb{Q}$. Portanto $\bigcap\left\{\Omega_{\alpha_{i}} \cap X: i \in \mathbb{N}\right\}=\mathbb{Q}$ e assim se tomarmos $\kappa=\bigcup\left\{\alpha_{i}: i \in \mathbb{N}\right\}$, então $y_{\kappa} \in \Omega_{\alpha_{i}} \forall i \in \mathbb{N}$ (pois $\alpha_{i} \leq \kappa$ e $\left.y_{\alpha} \in \bigcap\left\{\Omega_{\beta}: \beta \leq \kappa\right\}\right)$. Mas isso implicaria que $y_{\kappa} \in \mathbb{Q}$, o que é absurdo. Portanto $Y$ não é $F_{\sigma}$ em $X$.

Consideremos $A=\mathbb{Q} \times(\mathbb{R} \backslash \mathbb{Q})$ e $B=\{(y, y): y \in Y\}$ conjuntos fechados e disjuntos em $X \times(\mathbb{R} \backslash \mathbb{Q})$. Mostremos que eles não podem ser separados por abertos disjuntos em $X \times(\mathbb{R} \backslash \mathbb{Q})$.

Sejam $V$ e $U$ abertos quaisquer em $X \times(\mathbb{R} \backslash \mathbb{Q})$ tais que $A \subseteq V$ e $B \subseteq U$. Consideremos para cada $k \in \mathbb{N}^{+}$o conjunto $U_{k}=\left\{y \in Y:\{y\} \times S_{1 / k}(y) \subseteq U\right\}$, logo o conjunto $\left\{U_{k}: k \in \mathbb{N}^{+}\right\}$é recobrimento de $Y$ e pela afirmação, como $Y$ não é $F_{\sigma}$ em $X$, temos que existe $k \in \mathbb{N}^{+}$tal que $c l_{X}\left(U_{k}\right) \cap \mathbb{Q} \neq \phi$. Seja $q \in c l_{X}\left(U_{k}\right) \cap \mathbb{Q}$ e tomemos $y \in \mathbb{R} \backslash \mathbb{Q}$ tal que $|q-y|<1 / 2 k$. Então $(q, y) \in A$ e vamos mostrar que toda vizinhança aberta deste ponto intersecta $U$, assim mostraremos que $A$ e $B$ não podem ser separados por abertos disjuntos em $X \times(\mathbb{R} \backslash \mathbb{Q})$.

Suponhamos que $(q, y) \in M \times N \subseteq X \times(\mathbb{R} \backslash \mathbb{Q})$, onde $M$ é aberto em $X$ e $N$ é aberto em $(\mathbb{R} \backslash \mathbb{Q})$. Como $q \in c_{X}\left(U_{k}\right)$, existe $y^{\prime} \in M \cap U_{k}$ tal que $\left|q-y^{\prime}\right|<1 / 2 k$ assim: $\left(y^{\prime}, y\right) \in M \times N \mathrm{e}$ $\left|y^{\prime}-y\right| \leq\left|y^{\prime}-q\right|+|q-y|<\frac{1}{2 k}+\frac{1}{2 k}=\frac{1}{k} \Longrightarrow y \in S_{1 / k}\left(y^{\prime}\right) \Longrightarrow\left(y^{\prime}, y\right) \in\left\{y^{\prime}\right\} \times$ $S_{1 / k}\left(y^{\prime}\right) \subseteq U$, pois $y^{\prime} \in U_{k}$.

Ou seja $(M \times N) \cap U \neq \phi$, o que termina a demonstração.

No Teorema 2.3, foi usada a Hipótese do Contínuo para a construção do espaço topológico de Lindelöf e regular X (também normal). Em 1990, K. Alster [2], melhorou esse resultado usando o Axioma de Martin para mostrar que existe um espaço de Lindelöf $X$ tal que o espaço produto $X \times(\mathbb{R} \backslash \mathbb{Q})$ não é normal. A seguir, vamos estudar tal resultado. 
Primeiro vejamos algumas afirmações e alguns lemas que serão úteis:

Lembremos como foi definido " $<_{*}$ " em $\omega^{\omega}$ (Definição 0.5).

Se $x=(x(0), x(1), \ldots)$ e $y=(y(0), y(1), \ldots)$ são elementos em $\omega^{\omega}$, então dizemos que $x<_{*} y$ (ou respectivamente $x \leq_{*} y$ ) se existe $m<\omega$ tal que $x(i)<y(i)$ (ou respectivamente $x(i) \leq y(i))$, para todo $m<i<\omega$.

O Axioma de Martin implica que existe uma c-escala $S=\left\{x_{\alpha}: \alpha<\mathfrak{c}\right\}$ em $\omega^{\omega}$ (ver Corolário 0.16).

Até a prova do Teorema 2.7 (final da seção) vamos supor os seguintes fatos e fixar as seguintes notações:

- Vamos tomar os conjuntos $X_{\beta}$, para cada $\beta<\mathfrak{c}$, como sendo $X_{\beta}=\left\{x \in \omega^{\omega}\right.$ : $\left.x \leq_{*} x_{\beta}\right\}$ e para $\mathfrak{c}$, tomamos $X_{\mathfrak{c}}=(\omega+1)^{\omega}$.

- Consideraremos $\left((\omega+1)^{\omega}, d\right)$ como um espaço que é compactificação métrica de $\omega^{\omega}$.

- Tomaremos o espaço topológico

$$
(X, \Theta)
$$

como sendo o espaço no qual $X=(\omega+1)^{\omega}$ e $\Theta$ é a topologia na qual uma base para cada ponto de $X$ é dada da seguinte forma: se $x \in X_{\alpha} \backslash \bigcup\left\{X_{\beta}: \beta<\alpha\right\}$, onde $\alpha$ é o menor ordinal menor ou igual a c tal que $x \in X_{\alpha},\left((\omega+1)^{\omega}=X_{\mathfrak{c}}\right)$. Então

$$
\theta_{x}=\left\{\left(X_{\alpha} \cap W\right) \backslash X_{\beta}: W \text { é aberto em }(\omega+1)^{\omega} \text { contendo } x \text { e } \beta<\alpha\right\}
$$

Lema 2.4 (MA). Se $Z$ é um espaço compacto métrico tal que $Z=\bigcup\left\{Z_{\gamma}: \gamma<\beta\right\}$, onde $\beta<\mathfrak{c}$ e $Z_{\gamma}$ é compacto para cada $\gamma<\beta$, então existe uma sequência $\left(\gamma_{i}\right)_{i \in \mathbb{N}}$ em $\beta$ tal que $Z=\bigcup\left\{Z_{\gamma_{i}}: i \in \mathbb{N}\right\}$. 
Dem: Suponhamos que a tese do lema não é verdadeira. Vamos construir por recursão transfinita uma sequência estritamente crescente $\left\{H_{\gamma}: \gamma<\omega_{1}\right\}$ de conjuntos abertos em $Z$ de tal modo que o subespaço $L=\bigcup\left\{H_{\gamma}: \gamma<\omega_{1}\right\}$ não será de Lindelöf, o que é um absurdo, pois $Z$ é hereditariamente de Lindelöf por ter base enumerável de abertos. Tomemos

$$
H_{0}=\bigcup\left\{i n t_{Z} Z_{\gamma}: \gamma<\beta\right\}
$$

Temos que $H_{0} \neq \phi$, pois senão para cada $\gamma<\beta$ teríamos que $i n t_{Z} Z_{\gamma}=\phi$ e assim $Z$ seria um compacto e $T_{2}$ (que satisfaz c.c.c por ser separável) que é união de um número menor que $\mathfrak{c}$ de fechados raros, o que pelo Corolário 0.14 é absurdo.

Por outro lado, $H_{0}$ é de Lindelöf, pois $Z$ é hereditariamente de Lindelöf. Logo existe $T\left(H_{0}\right) \subseteq \beta$ enumerável tal que $H_{0}=\bigcup\left\{i n t_{Z} Z_{\gamma}: \gamma \in T\left(H_{0}\right)\right\} \subseteq \bigcup\left\{Z_{\gamma}: \gamma \in T\left(H_{0}\right)\right\}$.

Para cada $n$, tal que $0<n<\omega$, suponhamos definidos subconjuntos abertos não vazios $H_{0}, H_{1}, \ldots, H_{n-1}$ de $Z$ e subconjuntos $T\left(H_{0}\right), T\left(H_{1}\right), \ldots, T\left(H_{n-1}\right)$ de $\beta$ enumeráveis tais que:

(i) $H_{i-1} \subseteq H_{i}$ para cada $1 \leq i \leq n-1$

(ii) $H_{i} \subseteq \bigcup\left\{Z_{\gamma}: \gamma \in T\left(H_{i}\right)\right\}$, para cada $0 \leq i \leq n-1$

(iii) $H_{i} \backslash\left(\bigcup\left\{H_{t}: 0 \leq t<i\right\}\right) \neq \phi$, para cada $1 \leq i \leq n-1$

Vamos determinar $H_{n}$ e $T\left(H_{n}\right)$.

Tomemos

$$
H^{(n)}=\bigcup\left\{H_{t}: 0 \leq t \leq n-1\right\} \text { e } A^{(n)}=Z \backslash H^{(n)}
$$

$A^{(n)} \neq \phi$, pois senão $Z \subseteq H^{(n)}$ e teríamos que a tese do teorema vale. Seja

$$
H_{n}^{*}=\bigcup\left\{i n t_{A^{(n)}}\left(Z_{\gamma} \backslash H^{(n)}\right): \gamma<\beta\right\}
$$

Afirmamos que $H_{n}^{*} \neq \phi$. De fato, se for vazio, para cada $\gamma<\beta$, teríamos que

$$
i n t_{A^{(n)}}\left(Z_{\gamma} \backslash H^{(n)}\right)=\phi \text {. }
$$


Assim $A^{(n)}=\bigcup\left\{Z_{\gamma} \backslash H^{(n)}: \gamma<\beta\right\}$ seria um compacto, $T_{2}$ e que satisfaz c.c.c. que é união de um número menor que $\mathfrak{c}$ de conjuntos fechados e raros em $A^{(n)}$, que pelo Corolário 0.14 é absurdo. Além disso, como $H_{n}^{*}$ é de Lindelöf, existe $T\left(H_{n}^{*}\right) \subseteq \beta$ enumerável tal que $H_{n}^{*}=\bigcup\left\{i n t_{A^{(n)}}\left(Z_{\gamma} \backslash H^{(n)}\right): \gamma \in T\left(H_{n}^{*}\right)\right\}$.

Seja

$$
H_{n}=H_{n}^{*} \cup H^{(n)}
$$

Então,

1. $H_{n}$ é aberto em $Z$.

Com efeito, $Z \backslash H_{n}=\left(Z \backslash H_{n}^{*}\right) \cap\left(Z \backslash H^{(n)}\right)$. Como $H_{n}^{*}$ é aberto em $A^{(n)}$, existe $W \subseteq Z$ aberto tal que $H_{n}^{*}=W \cap A^{(n)}$. Logo,

$Z \backslash H_{n}=\left(Z \backslash\left(W \cap A^{(n)}\right)\right) \cap A^{(n)}=\left((Z \backslash W) \cup\left(Z \backslash A^{(n)}\right)\right) \cap A^{(n)}=(Z \backslash W) \cap A^{(n)}$.

Portanto $Z \backslash H_{n}$ é fechado em $Z$.

2. Vale que $H_{n-1} \subseteq H_{n}$, pois $H_{n}=H_{n}^{*} \cup H^{(n)}$.

3. Existência de $T\left(H_{n}\right)$.

Tomemos $T\left(H_{n}\right)=T\left(H_{n}^{*}\right) \cup \bigcup\left\{T\left(H_{t}\right): 0 \leq t \leq n-1\right\}$. Assim, $H_{n} \subseteq \bigcup\left\{Z_{\gamma}: \gamma \in\right.$ $\left.T\left(H_{n}\right)\right\}$ e vale a Condição (ii), para $i=n$.

4. $H_{n}^{*}=H_{n} \backslash \bigcup\left\{H_{\gamma}: 0 \leq n-1\right\}$, pois $H_{n}=H_{n}^{*} \cup H^{(n)}$ e $H_{n}^{*} \cap H^{(n)}=\phi$. Dado que $H_{n}^{*} \neq \phi$ temos que vale a Condição (iii), para $i=n$.

Suponhamos que para cada número ordinal $\alpha$, com $\alpha<\delta\left(\operatorname{com} \delta<\omega_{1}\right)$, temos definidos subconjuntos abertos não vazios $H_{\alpha}$ 's de $Z$ e subconjuntos $T\left(H_{\alpha}\right)$ 's de $\beta$ enumeráveis tais que:

(I) $H_{\alpha_{1}} \subseteq H_{\alpha_{2}}$, para cada $\alpha_{1}<\alpha_{2}<\delta$

(II) $H_{\alpha} \subseteq \bigcup\left\{Z_{\alpha^{\prime}}: \alpha^{\prime} \in T\left(H_{\alpha}\right)\right\}$, para cada $\alpha<\delta$

(III) $H_{\alpha} \backslash\left(\bigcup\left\{H_{\alpha^{\prime}}: \alpha^{\prime}<\alpha\right\}\right) \neq \phi$, para cada $\alpha<\delta$ 
Vamos determinar $H_{\delta}$ e $T\left(H_{\delta}\right)$

Tomemos

$$
H^{(\delta)}=\bigcup\left\{H_{\gamma}: \gamma<\delta\right\} \text { e } A^{(\delta)}=Z \backslash H^{(\delta)}
$$

$A^{(\delta)} \neq \phi$, pois senão $Z \subseteq H^{(\delta)}$ e teríamos que a tese do teorema vale. Seja

$$
H_{\delta}^{*}=\bigcup\left\{i n t_{A^{(\delta)}}\left(Z_{\gamma} \backslash H^{(\delta)}\right): \gamma<\beta\right\}
$$

Afirmamos que $H_{\delta}^{*} \neq \phi$. De fato, senão para cada $\gamma<\beta$, teríamos que $i n t_{A^{(\delta)}}\left(Z_{\gamma} \backslash H^{(\delta)}\right)=$ $\phi$. Assim $A^{(\delta)}=\bigcup\left\{Z_{\gamma} \backslash H^{(\delta)}: \gamma<\beta\right\}$ seria um compacto, $T_{2}$ e que satisfaz c.c.c. que é união de um número menor que $\mathfrak{c}$ de conjuntos fechado raros em $A^{(\delta)}$, o que pelo Corolário 0.14 é absurdo. Além disso, como $H_{\delta}^{*}$ é de Lindelöf, existe $T\left(H_{\delta}^{*}\right) \subseteq \beta$ enumerável tal que $H_{\delta}^{*}=\bigcup\left\{i n t_{A^{(\delta)}}\left(Z_{\gamma} \backslash H^{(\delta)}\right): \gamma \in T\left(H_{\delta}^{*}\right)\right\}$.

Seja

$$
H_{\delta}=H_{\delta}^{*} \cup H^{(\delta)} .
$$

Do mesmo modo que mostramos que $H_{n}$ satisfaz as condições (i), (ii) e (iii), podemos mostrar que $H_{\delta}$ satistaz

- $H_{\alpha_{1}} \subseteq H_{\delta}$, para cada $\alpha_{1}<\delta$

- $H_{\delta} \subseteq \bigcup\left\{Z_{\alpha^{\prime}}: \alpha^{\prime} \in T\left(H_{\delta}\right)\right\}$.

- $H_{\delta} \backslash\left(\bigcup\left\{H_{\alpha^{\prime}}: \alpha^{\prime}<\delta\right\}\right) \neq \phi$.

Portanto temos o conjunto $\left\{H_{\gamma}: \gamma<\omega_{1}\right\}$ com as condições que queríamos.

Lema 2.5 (MA). Se $X_{\alpha}^{\prime}=X_{\alpha} \backslash \bigcup\left\{X_{\beta}: \beta<\alpha\right\}$ e U é um aberto em $(\omega+1)^{\omega}$ contendo $X_{\alpha}^{\prime}$, então existe uma sequência $\left(\gamma_{i}\right)_{i \in \mathbb{N}}$ em $\alpha$ tal que $X_{\alpha} \backslash U \subseteq \bigcup\left\{X_{\gamma_{i}}: i \in \mathbb{N}\right\}$.

Dem: Afirmação: cada subespaço $X_{\alpha}^{\prime}$ de $X$ é de Lindelöf.

De fato, suponhamos que

$$
X_{\alpha}^{\prime} \subseteq \bigcup\left\{\left(X_{\alpha} \cap W_{i}\right) \backslash X_{\beta_{i}}: i \in I\right\}
$$


onde para cada $i \in I, W_{i} \subseteq(\omega+1)^{\omega}$ é aberto e $\beta_{i}$ é um ordinal tal que $\beta_{i}<\alpha$. Logo, como $(\omega+1)^{\omega}$ é hereditariamente de Lindelöf, (pois é separável e metrizável) existe $J \subseteq I$ enumerável, tal que $\bigcup\left\{W_{i}: i \in I\right\}=\bigcup\left\{W_{i}: i \in J\right\}$.

Vejamos que

$$
X_{\alpha}^{\prime} \subseteq \bigcup\left\{\left(X_{\alpha} \cap W_{r}\right) \backslash X_{\beta_{r}}: r \in J\right\} .
$$

Com efeito, se $x \in X_{\alpha}^{\prime}$ (notemos que se $x \in X_{\alpha}^{\prime}$, então $x \notin X_{\beta}$ para cada $\beta<\alpha$ ) assim existe $k \in J$ tal que $x \in X_{\alpha} \cap W_{k}$ e $x \notin X_{\beta_{k}}$. Portanto $x \in\left(X_{\alpha} \cap W_{k}\right) \backslash X_{\beta_{k}}$, ou seja $X_{\alpha}^{\prime} \subseteq \bigcup\left\{\left(X_{\alpha} \cap W_{r}\right) \backslash X_{\beta_{r}}: r \in J\right\}$. Portanto $X_{\alpha}^{\prime}$ é de Lindelöf para cada $\alpha \leq \mathfrak{c}$.

Para mostrar o lema, vamos a dividir a demonstração em dois casos: primeiro mostraremos que vale se $c f(\alpha)=\omega$ e depois para $c f(\alpha)>\omega$.

Se $c f(\alpha)=\omega$, temos que existe uma função $f: \omega \longrightarrow \alpha$, tal que $f[\omega]$ é cofinal em $\alpha$. Vamos mostrar que $X_{\alpha} \backslash U \subseteq \bigcup\left\{X_{f(\lambda)}: \lambda<\omega\right\}$.

$$
\begin{aligned}
x \in X_{\alpha} \backslash U & \Longrightarrow x \leq_{*} x_{\alpha} \text { e } x \notin U \\
& \Longrightarrow x \leq_{*} x_{\alpha} \text { e } x \notin X_{\alpha}^{\prime} \\
& \left.\Longrightarrow x \leq_{*} x_{\alpha} \text { e } x \in \bigcup\left\{X_{\beta}: \beta<\alpha\right\}\right) \\
& \Longrightarrow \text { existe um ordinal } \beta, \text { com } \beta<\alpha, \text { tal que } x \in X_{\beta} \\
& \Longrightarrow x \in X_{\beta} \text { e existe } \lambda<\omega \text { tal que } \beta<f(\lambda)<\alpha \\
& \Longrightarrow x \leq_{*} x_{\beta}<_{*} x_{f(\lambda)} \\
& \Longrightarrow x \in \bigcup\left\{X_{f(\lambda)}: \lambda<\omega\right\} .
\end{aligned}
$$

Agora suponhamos que $\omega<c f(\alpha)$.

Vamos mostrar que podemos tomar $U$ como sendo um aberto da forma $\left(X_{\alpha} \cap\right.$ $H) \backslash X_{\beta_{1}}$ onde $\beta_{1}$ é um ordinal tal que $\beta_{1}<\alpha$ e $H$ é um aberto em $(\omega+1)^{\omega}$.

De fato, para cada $x \in X_{\alpha}^{\prime}$, existe $U_{x} \subseteq(\omega+1)^{\omega}$ aberto e um ordinal $\beta_{x}$, com $\beta_{x}<\alpha$, tal que $x \in\left(X_{\alpha} \cap U_{x}\right) \backslash X_{\beta_{x}} \subseteq U$. Então $X_{\alpha}^{\prime} \subseteq \bigcup\left\{\left(X_{\alpha} \cap U_{x}\right) \backslash X_{\beta_{x}}: x \in X_{\alpha}^{\prime}\right\} \subseteq U$. Agora, pela afirmação, temos que existe um conjunto $I \subseteq X_{\alpha}^{\prime}$ enumerável tal que $X_{\alpha}^{\prime} \subseteq \bigcup\left\{\left(X_{\alpha} \cap U_{x}\right) \backslash X_{\beta_{x}}: x \in I\right\} \subseteq U$. 
Seja $A=\left\{\beta_{x}: x \in I\right\}$. Como $\omega<c f(\alpha)$, então $A$ não é cofinal em $\alpha$. Logo, existe um ordinal $\bar{\beta}$, com $\bar{\beta}<\alpha$, tal que $\beta_{x}<\bar{\beta}$ (portanto $X_{\beta_{x}} \subseteq X_{\bar{\beta}}$ ) para cada $x \in I$. Portanto,

$\bigcup\left\{\left(X_{\alpha} \cap U_{x}\right) \backslash X_{\bar{\beta}}: x \in I\right\} \subseteq \bigcup\left\{\left(X_{\alpha} \cap U_{x}\right) \backslash X_{\beta_{x}}: x \in I\right\} \subseteq U$.

Vejamos que $X_{\alpha}^{\prime} \subseteq \bigcup\left\{\left(X_{\alpha} \cap U_{x}\right) \backslash B_{\bar{\beta}}: x \in I\right\}$. Se $x \in X_{\alpha}^{\prime}$, então existe $y \in I$ tal que $x \in\left(X_{\alpha} \cap U_{y}\right) \backslash X_{\beta_{y}}, \operatorname{logo} x \in X_{\alpha}, x \in U_{y}$ e $x \notin X_{\beta_{y}}$. Na verdade, como $x \in X_{\alpha}^{\prime}$, então $x \notin X_{\beta}$ para todo $\left.\beta<\alpha\right)$. Assim $x \in X_{\alpha}, x \in U_{y}$ e $x \notin X_{\bar{\beta}}$. Então $x \in\left(X_{\alpha} \cap U_{y}\right) \backslash X_{\bar{\beta}}$.

Se nós tomarmos $H=\bigcup\left\{U_{x}: x \in I\right\}$, então temos que $H$ é um aberto em $(\omega+1)^{\omega}$ tal que $X_{\alpha}^{\prime} \subseteq\left(X_{\alpha} \cap H\right) \backslash X_{\bar{\beta}} \subseteq U$.

Doravante na prova do lema, para o caso $c f(\alpha)>\omega$, supomos $U=\left(X_{\alpha} \cap H\right) \backslash X_{\beta_{1}}$, onde $H$ é um aberto em $(\omega+1)^{\omega}$ e $\beta_{1}<\alpha$.

Caso 1: Se $\alpha=\mathfrak{c}$.

Como $X_{\mathfrak{c}} \backslash H \subseteq(\omega+1)^{\omega}$ é fechado, ele é compacto. Além disso $X_{\mathfrak{c}} \backslash H \subseteq \omega^{\omega}$, pois $X_{\mathfrak{c}} \backslash H \subseteq \bigcup\left\{X_{\beta}: \beta<\mathfrak{c}\right\}=\omega^{\omega}$. Assim, para cada $n<\omega$ temos que $\prod_{n}\left[X_{\mathfrak{c}} \backslash H\right]$ é compacto em $\omega$, logo existe $y(n)<\omega$ tal que se $x \in \prod_{n}\left[X_{\mathfrak{c}} \backslash H\right]$ então $x \leq y(n)$. Portanto existe uma sequência $y=(y(i))_{i \in \mathbb{N}}$ em $\omega^{\omega}$ tal que

$$
X_{\mathfrak{c}} \backslash H \subseteq\left\{(x(n))_{n \in \mathbb{N}} \in \omega^{\omega}: x(n) \leq y(n), \forall n<\omega\right\}=K
$$

Como $S=\left\{x_{\beta}: \beta<\mathfrak{c}\right\}$ é uma c-escala em $\omega^{\omega}$, existe $\beta_{2}<\mathfrak{c}$, tal que $y<_{*} x_{\beta_{2}}$, o que implica que $K \subseteq X_{\beta_{2}}$. Portanto se nós mostrarmos que $X_{\mathfrak{c}} \backslash U \subseteq X_{\beta_{1}} \cup X_{\beta_{2}}$ terminará a demonstração do lema no caso 1 . De fato, se

$$
\begin{aligned}
x \in X_{\mathfrak{c}} \backslash U & \Longrightarrow x \in X_{\mathfrak{c}} \text { e } x \notin\left(X_{\mathfrak{c}} \cap H\right) \backslash X_{\beta_{1}} \\
& \Longrightarrow x \in X_{\mathfrak{c}} \text { e }\left(x \notin H \text { ou } x \in\left(H \cap X_{\beta_{1}}\right)\right) \\
& \Longrightarrow x \in\left(X_{\mathfrak{c}} \backslash H\right) \cup\left(X_{\mathfrak{c}} \cap\left(H \cap X_{\beta_{1}}\right)\right) \subseteq K \cup X_{\beta_{1}} \subseteq X_{\beta_{2}} \cup X_{\beta_{1}}
\end{aligned}
$$

o que implica que $X_{\mathfrak{c}} \backslash U \subseteq X_{\beta_{1}} \cup X_{\beta_{2}}$. 
Caso 2: Se $\alpha<\mathfrak{c}$.

Vejamos que para cada $\beta<\alpha, X_{\beta}$ é um $\sigma$-compacto em $\omega^{\omega}$. De fato, se tomamos para cada $n<\omega$,

$$
V_{n}=\underbrace{\omega \times \cdots \times \omega}_{\mathrm{n}+1 \text {-vezes }} \times\left\{0,1, \ldots, x_{\beta}(n+1)\right\} \times\left\{0,1, \ldots, x_{\beta}(n+2)\right\} \times \cdots
$$

onde $x_{\beta}=\left(x_{\beta}(0), x_{\beta}(1), \ldots\right)$, então cada $V_{n}$ seria $\sigma$-compacto em $\omega^{\omega}$. Logo $X_{\beta}=\bigcup\left\{V_{n}\right.$ : $n<\omega\}$ é $\sigma$-compacto em $\omega^{\omega}$.

Vamos supor que $X_{\beta}=\bigcup\left\{X_{\beta}(n): n<\omega\right\}$, onde para cada $n<\omega$, temos que $X_{\beta}(n)$ é compacto em $\omega^{\omega}$. Então,

$$
X_{\alpha} \backslash H \subseteq \bigcup\left\{X_{\gamma}: \gamma<\alpha\right\}=\bigcup\left\{X_{\gamma}(m): m<\omega \text { e } \gamma<\alpha\right\}
$$

Seja $D_{n}=X_{\alpha}(n) \backslash H$. Então $D_{n}=\bigcup\left\{\left(X_{\alpha}(n) \backslash H\right) \cap X_{\gamma}(m): \gamma<\alpha\right.$ e $\left.m<\omega\right\}$ para cada $n<\omega$. Agora, como $D_{n}$ é um subconjunto compacto de $\omega^{\omega}$, que é união de um número menor que $\mathfrak{c}$ de subconjuntos compactos, pelo Lema 2.4, temos que existe uma sequência $\left(\gamma_{i}(n)\right)_{i \in \mathbb{N}}$ contida em $\alpha$, tal que $D_{n}=\bigcup\left\{\left(X_{\alpha}(n) \backslash H\right) \cap X_{\gamma_{i}(n)}(m): i<\omega\right.$ e $m<\omega\}$. Seja $\left\{\gamma_{j}: i \in \mathbb{N}\right\}$ uma reindexação do conjunto $\bigcup\left\{\gamma_{i}(n): n, i<\omega\right\}$.

Nestas condições afirmamos que $X_{\alpha} \backslash U \subseteq \bigcup\left\{X_{\gamma_{i}}: i<\omega\right\} \cup\left\{X_{\beta_{1}}\right\}$. De fato,

$$
\begin{aligned}
x \in X_{\alpha} \backslash U & \Longrightarrow x \in X_{\alpha} \text { e } x \notin\left(X_{\alpha} \cup H\right) \backslash X_{\beta_{1}} \\
& \Longrightarrow x \in X_{\alpha} \text { e }\left(x \notin H \text { ou } x \in\left(H \cap X_{\beta_{1}}\right)\right) \\
& \Longrightarrow x \in\left(X_{\alpha} \backslash H\right) \cup X_{\beta_{1}} \\
& \Longrightarrow \text { existe } k<\omega \text { tal que } x \in D_{k} \cup X_{\beta_{1}} \\
& \Longrightarrow \text { existe } j<\omega \text { tal que } x \in X_{\gamma_{j}} \cup X_{\beta_{1}}
\end{aligned}
$$

isto termina a demonstração do Lema 2.5.

Lema 2.6 (MA). Para cada $\alpha \leq \mathfrak{c}$, o espaço $X_{\alpha}$ é de Lindelöf.

Dem: Por indução, observemos que $X_{0}$ é um espaço de Lindelöf, pois a topologia dele é a mesma que herda do espaço $(\omega+1)^{\omega}$, que é um espaço hereditariamente de Lindelöf. 
Suponhamos (hipótese de indução) que para cada $\alpha<\beta$, o espaço $X_{\alpha}$ é de Lindelöf e mostremos que $X_{\beta}$ também é de Lindelöf. Seja

$$
\mathcal{U}=\left\{U_{s}: s \in S\right\}
$$

um recobrimento aberto de $X_{\beta}$ em $(\omega+1)^{\omega}$. Dado que $X_{\beta}^{\prime}$ é de Lindelöf (por uma afirmação feita no Lema 2.5), temos que existe $S^{*} \subseteq S$ enumerável, tal que $X_{\beta}^{\prime} \subseteq \bigcup\left\{U_{s}\right.$ : $\left.s \in S^{*}\right\}=U$. Pelo Lema 2.5, existe $\left(\gamma_{i}\right)_{i \in \mathbb{N}}$ em $\beta$, tal que $X_{\beta} \backslash U \subseteq \bigcup\left\{X_{\gamma_{i}}: i \in \mathbb{N}\right\}$. Mas pela hipótese de indução temos que para cada $i \in \mathbb{N}$, existe um conjunto $S_{i} \subseteq S$ enumerável, tal que $X_{\gamma_{i}}=\bigcup\left\{X_{\gamma_{i}} \cap U_{s}: s \in S_{i}\right\}$. Portanto, se tomarmos

$$
T=\bigcup\left\{S_{i}: i \in \mathbb{N}\right\} \cup S^{*},
$$

então $T$ é enumerável e $X_{\beta} \subseteq \bigcup\left\{U_{s}: s \in T\right\}$. Assim, $X_{\beta}$ é de Lindelöf.

Teorema 2.7 (MA). Existe um espaço topológico de Lindelöf (regular) tal que o seu produto com o subespaço $\mathbb{R} \backslash \mathbb{Q}$ não é normal.

Dem: Como sabemos que $\mathbb{R} \backslash \mathbb{Q}$ é homeomorfo ao $\omega^{\omega}$, vamos mostrar que o espaço topológico $(X, \Theta)$ como em (2.2.1) é um espaço de Lindelöf tal que $X \times \omega^{\omega}$ não é normal. Pelo Lema 2.2, como $X=X_{\mathfrak{c}}$, temos que $X$ é um espaço de Lindelöf. Vamos mostrar agora que $X \times \omega^{\omega}$ não é de Lindelof e portanto não vai ser normal (ver Teorema $3.4)$.

Em um espaço de Lindelöf, todo subconjunto fechado é de Lindelöf. Assim, para mostrar que $X \times \omega^{\omega}$ não é de Lindelöf vamos apresentar um subconjunto fechado dele que não é de Lindelöf. Para isso tomemos

$$
F=\left\{(p, p): p \in \omega^{\omega}\right\}
$$

um subconjunto fechado em $X \times \omega^{\omega}$, e consideremos a família

$$
\mathcal{V}=\left\{F \cap\left(X_{\alpha} \times \omega^{\omega}\right): \alpha<\mathfrak{c}\right\}
$$


1. $\mathcal{V}$ é um recobrimento aberto de $F$.

Com efeito, por indução transfinita veremos que $X_{\alpha}$ é aberto em $X$ para cada $\alpha<\mathfrak{c}$. Claramente temos que $X_{0}$ é aberto em $X$. Seja $\beta<\mathfrak{c}$ e suponhamos que para cada $\alpha<\beta, X_{\alpha}$ é aberto em $X$; vamos mostrar que $X_{\beta}$ é aberto em $X$. Seja $x \in X_{\beta}$. Consideremos dois casos:

(a) Caso 1: Se existe $\alpha_{1}<\beta$, tal que $x \in X_{\alpha_{1}}$, então pela hipótese de indução, $x$ é um ponto interior em $X_{\beta}$.

(b) Caso 2: Se $x \notin \bigcup\left\{X_{\alpha}: \alpha<\beta\right\}$, então $x \in X_{\beta}^{\prime}$, logo como $\left(X_{\beta} \cap X\right) \backslash X_{\lambda}$ é um aberto, que pertence à base de $x$ definida em (2.2.2), contido em $X_{\beta}$, para cada $\lambda<\beta$, temos que $x$ é um ponto interior em $X_{\beta}$.

É claro que $F \subseteq \cup \mathcal{V}$, portanto concluímos que $\mathcal{V}$ é um recobrimento aberto de $F$.

\section{2. $\mathcal{V}$ não tem sub-recobrimento enumerável.}

Com efeito, se existir $M \subseteq \mathfrak{c}$ enumerável tal que $\left\{F \cap\left(X_{\alpha} \times \omega^{\omega}\right): \alpha \in M\right\}$ seja sub-recobrimento de $\mathcal{V}$, então $\lambda=\bigcup M$ seria um ordinal menor que $\mathfrak{c}$ (pois a cofinalidade de $\mathfrak{c}$ é não enumerável). Assim existe um ordinal $\bar{\alpha}, \operatorname{com} \lambda<\bar{\alpha}<\mathfrak{c}$. Logo existe $\left(x_{\bar{\alpha}}, x_{\bar{\alpha}}\right)$ em $\omega^{\omega}, x_{\bar{\alpha}}$ na c-escala $S$, que não pertence a $\left\{F \cap\left(X_{\alpha} \times \omega^{\omega}\right)\right.$ : $\alpha \in M\}$ absurdo.

Portanto $F$ não é de Lindelöf e com isso terminamos a demonstração do teorema.

\subsection{Concentração e produto de espaços de Lindelöf com os irracionais}

Nesta seção vamos estudar o artigo de Lawrence [10]. Vamos introduzir o conceito de concentração que tem aplicações no produto de espaços de Lindelöf com o subespaço 
$\mathbb{R} \backslash \mathbb{Q}$. Nós veremos quando um espaço topológico é concentrado num subconjunto dele. Nestas condições é possível encontrar exemplos de espaços métricos separáveis e espaços de Lindelöf cujo produto não é normal. Se assumimos que $\mathfrak{b}=\omega_{1}$ é possível encontrar um exemplo onde o espaço métrico separável pode ser $\mathbb{R} \backslash \mathbb{Q}$.

No final desta seção apresentaremos um resultado que dá uma condição para que produto de espaços de Lindelöf por espaços completamente metrizáveis e separáveis seja normal.

Definição 2.2. Seja $X$ um espaço topológico e $A \subseteq X$. Dizemos que $X$ é concentrado em $A$ se para todo aberto $U$ contendo $A, X \backslash U$ é enumerável.

Lembremos que $\mathbb{M}$, neste trabalho, denota a reta de Michael; isto é, $\mathbb{M}$ é o conjunto $\mathbb{R}$ com a topologia:

$$
\Theta_{\mathbb{M}}=\{V \cup W: V \text { é um aberto em } \mathbb{R} \text { e } W \subseteq \mathbb{R} \backslash \mathbb{Q}\}
$$

Também lembremos um pouco sobre subconjuntos compacto e $\sigma$-compactos de $\omega^{\omega}$.

Para cada $f \in \omega^{\omega}$, definimos $C_{f} \subseteq \omega^{\omega}$, como segue:

$$
C_{f}=\left\{g \in \omega^{\omega}: g \leq f\right\}
$$

É fácil ver que $C_{f}=\prod_{i<\omega}[0, f(i)]$, onde $[0, f(i)]$ é um intervalo considerado em $\omega$ para cada $i<\omega$. Assim cada $C_{f}$ vai ser um subconjunto compacto.

Notemos também que se $C$ é um subconjunto compacto de $\omega^{\omega}$, então existe $f \in \omega^{\omega}$ tal que $C \subseteq C_{f}$. Definamos para cada $f \in \omega^{\omega}$,

$$
C_{f}^{*}=\left\{g \in \omega^{\omega}: g \leq_{*} f\right\}
$$

onde $\leq_{*}$ é definido como na definição 0.5.

Vejamos que para cada $f \in \omega^{\omega}, C_{f}^{*}$ é $\sigma$-compacto. De fato, se tomarmos para cada $n<\omega$,

$$
W_{n}=\underbrace{\omega \times \cdots \times \omega}_{(n+1) \text {-vezes }} \times\{0,1, \ldots, f(n+1)\} \times\{0,1, \ldots, f(n+2)\} \times \cdots,
$$


então cada $W_{n}$ é $\sigma$-compacto e como $C_{f}^{*}=\bigcup\left\{W_{n}: n<\omega\right\}$, tenemos que $C_{f}^{*}$ é $\sigma$ compacto.

Além disso, se $K \subseteq \omega^{\omega}$ é $\sigma$-compacto, então existe $g \in \omega^{\omega}$ tal que $K \subseteq C_{g}^{*}$. De fato, como $K$ é $\sigma$-compacto, pela observação acima, existe uma família $\left\{g_{k} \in \omega^{\omega}: k<\omega\right\}$ tal que $K \subseteq \bigcup\left\{C_{g_{k}}: k<\omega\right\}$. Tomando $g \in \omega^{\omega}$ de tal modo que para cada $n<\omega$, $g(n)=\sum_{k \leq n} g_{k}(n)$, segue-se que $K \subseteq C_{g}^{*}$.

Vamos agora enunciar alguns resultados para mostrar o Teorema 2.10, onde apresentamos outro exemplo, supondo que $\mathfrak{b}=\omega_{1}$, de um espaço normal e de Lindelöf $X$ tal que $X \times(\mathbb{R} \backslash \mathbb{Q})$ não é normal.

Lema 2.8. Seja $X$ um subconjunto não enumerável de $\omega^{\omega}$. Então são equivalentes as seguintes afirmações:

i) Todo subconjunto não enumerável de X é ilimitado em $\left(\omega^{\omega}, \leq_{*}\right)$.

ii) X é um subconjunto não enumerável de $\omega^{\omega}$ que tem intersecção enumerável com $C_{g}^{*}$ para cada $g \in \omega^{\omega}$.

iii) X tem intersecção enumerável com qualquer subconjunto compacto de $\omega^{\omega}$.

iv) $X \cup \mathbb{Q}$, como subespaço de $\mathbb{R}$, é um espaço concentrado em $\mathbb{Q}$.

v) $X \cup \mathbb{Q}$, como subespaço de $\mathbb{M}$, é um espaço de Lindelöf.

Dem: Mostremos primeiramente que i) e iii) são equivalentes a ii).

i) $\rightarrow$ ii): Suponhamos que $X$ possui intersecção não enumerável com $C_{g}^{*}$ para algum $g \in \omega^{\omega}$. Então $X \cap C_{g}^{*}$ seria um subconjunto de $X$ não enumerável e limitado em $\left(\omega^{\omega}, \leq_{*}\right)$, que contradiz i).

ii) $\rightarrow$ i): Seja $B \subseteq X$ não enumerável e suponhamos que $B$ é limitado. Então existe 
$f \in \omega^{\omega}$ tal que $B \subseteq C_{f}^{*}$; $\log C_{f}^{*} \cap X$ seria não enumerável, o que contradiz ii).

ii) $\rightarrow$ iii): Seja $K$ um subconjunto compacto de $\omega^{\omega}$. Então existe $g \in \omega^{\omega}$ tal que $K \subseteq C_{g}^{*}$, portanto como $X \cap C_{g}^{*}$ é enumerável, temos que $X \cap K$ é também enumerável.

iii) $\rightarrow$ ii): Seja $g \in \omega^{\omega}$. Sabemos que $C_{g}^{*}$ é $\sigma$-compacto. Portanto, como a união enumerável de conjuntos enumeráveis é enumerável e usando iii), temos que $X \cap C_{g}^{*}$ é enumerável.

Para mostrar a equivalência entre iii) e iv). Vamos usar os seguintes fatos:

- ([8]) Um subconjunto $K$ de $\mathbb{R} \backslash \mathbb{Q}$ é compacto em $\mathbb{R} \backslash \mathbb{Q}$ se e só se $K$ é um subconjunto fechado, limitado e de interior vazio em $\mathbb{R} \backslash \mathbb{Q}$ que também é fechado como subconjunto de $\mathbb{R}$.

- No é dificil mostrar que se $X$ é um espaço topológico, $F$ um subconjunto fechado de $X$ e $D$ um subconjunto denso de $X$, então $F$ tem interior vazio em $X$ se e só se $F \cap D$ tem interior vazio em $D$.

iii) $\rightarrow$ iv): Seja $W \subseteq X \cup \mathbb{Q}$ aberto qualquer contendo $\mathbb{Q}$. Então existe $W^{*}$ aberto de $\mathbb{R}$ tal que $W=W^{*} \cap(X \cup \mathbb{Q})$. Observemos que $(X \cup \mathbb{Q}) \backslash W=X \backslash W^{*}$, ou seja $X \backslash W^{*}$ é o complementar de $W$ em $X \cup \mathbb{Q}$. Assim para mostrar que $X \cup \mathbb{Q}$ é concentrado em $\mathbb{Q}$, basta mostrar que $X \backslash W^{*}$ é enumerável. Para isso note também que $X \backslash W^{*} \subseteq \mathbb{R} \backslash W^{*}$, onde $\mathbb{R} \backslash W^{*}$ é um fechado em $\mathbb{R}$ contido em $\mathbb{R} \backslash \mathbb{Q}$ e portanto $\sigma$-compacto em $\mathbb{R} \backslash \mathbb{Q}$. Segue-se que $X \backslash W^{*}$ é enumerável, pois do contrário possuiria intersecção não enumerável pelo menos com algum dos compactos de $\mathbb{R} \backslash \mathbb{Q}$ cuja reunião é $\mathbb{R} \backslash W^{*}$, o que contradiz iii). Portanto $X \cup \mathbb{Q}$ é concentrado em $\mathbb{Q}$.

iv) $\rightarrow$ iii): Suponhamos por absurdo que existe $K \subseteq \omega^{\omega}$ compacto tal que $X \cap K$ seja não enumerável. Então $\mathbb{R} \backslash K$ é um aberto em $\mathbb{R}$ contendo o conjunto $\mathbb{Q}$. Assim, $W=(\mathbb{R} \backslash K) \cap(X \cup \mathbb{Q})$ é um aberto de $X \cup \mathbb{Q}$ que contém $\mathbb{Q}$ e tal que $(X \cup \mathbb{Q}) \backslash W=X \cap K$ 
é não enumerável, o que contradiz iv). Portanto $X$ tem intersecção enumerável com qualquer subconjunto de $\omega^{\omega}$.

Para finalizar a demonstração nós mostraremos que iv) e v) são equivalentes:

iv) $\rightarrow \mathrm{v}$ ): Seja $\mathcal{U}=\left\{U_{i} \cup V_{i}: i \in I\right\}$ um recobrimento aberto em $\mathbb{M} \operatorname{de} X \cup \mathbb{Q}$, com $U_{i}$ aberto de $\mathbb{R}$ e $V_{i} \subseteq \mathbb{R} \backslash \mathbb{Q}$, para cada $i \in I$. Então existe $J \subseteq I$ enumerável tal que $\bigcup\left\{U_{i}: i \in J\right\}=\bigcup\left\{U_{i}: i \in I\right\}$. Mas $\bigcup\left\{U_{i}: i \in J\right\} \cap(X \cup \mathbb{Q})$ é um aberto em $X \cup \mathbb{Q}$ contendo $\mathbb{Q}$. Portanto, de iv) temos que $(X \cup \mathbb{Q}) \backslash \bigcup\left\{U_{i}: i \in J\right\}$ é enumerável. Logo existe um sub-recobrimento enumerável de $\mathcal{U}$. Portanto, $X \cup \mathbb{Q}$ como subespaço de $\mathbb{M}$ é de Lindelöf.

v) $\rightarrow$ iv): Seja $W \subseteq X \cup \mathbb{Q}$ aberto qualquer contendo $\mathbb{Q}$. Logo existe $W^{*} \subseteq \mathbb{R}$ aberto tal que $W=(X \cup \mathbb{Q}) \cap W^{*}$. Observemos que $(X \cup \mathbb{Q}) \backslash W=X \backslash W^{*}$. Assim se $(X \cup \mathbb{Q}) \backslash W$ fosse não enumerável, então $X \backslash W^{*}$ também será não enumerável. Portanto o conjunto $\left\{W^{*}\right\} \cup\left\{\{x\}: x \in X \backslash W^{*}\right\}$ seria um recobrimento aberto em $\mathbb{M}$ de $X \cup \mathbb{Q}$ que não tem sub-recobrimento enumerável, o que contradiz v). Então $X \cup \mathbb{Q}$ como subespaço de $\mathbb{R}$ é concentrado em $\mathbb{Q}$.

O lema seguinte dá uma condição necessária e suficiente para que exista um conjunto verificando as condições do lema anterior.

Lema 2.9. Existe um subconjunto não enumerável $X$ contido em $\mathbb{R} \backslash \mathbb{Q}$ satisfazendo uma (e portanto todas) das condições do Lema 2.8 se é só se $\mathfrak{b}=\omega_{1}$.

Dem: Se $X \subseteq \mathbb{R} \backslash \mathbb{Q}$ é não enumerável e satisfaz todas as condições do Lema 2.8, então tomamos $Y \subseteq X \operatorname{com}|Y|=\omega_{1}$. Assim a condição i) nos diz que $Y$ é ilimitado em $\left({ }^{\omega} \omega, \leq_{*}\right)$, de onde $\mathfrak{b}=\omega_{1}$. 
Por outro lado, se $\mathfrak{b}=\omega_{1}$, usamos a igualdade $\mathfrak{b}=\mathfrak{b}_{1}$ do Teorema 0.2 , e tomamos $X=\left\{f_{\beta}: \beta<\omega_{1}\right\}$ ilimitado em $\left({ }^{\omega} \omega, \leq_{*}\right)$, bem ordenado pela ordem $<^{*}$. Claramente $X$ satisfaz a condição i) do Lema 2.8 .

Teorema 2.10. Se $\mathfrak{b}=\omega_{1}$, então existe um espaço de Lindelöf e regular $X$ tal que o produto topológico $X \times(\mathbb{R} \backslash \mathbb{Q})$ não é normal.

Dem: Se $\mathfrak{b}=\omega_{1}$, então pelo Lema 2.9 existe $Z \subseteq \mathbb{R} \backslash \mathbb{Q}$ satisfazendo as condições do Teorema 2.8. Seja $X=Z \cup \mathbb{Q}$ com a topologia de subespaço de $\mathbb{M}$. É fácil ver que $X$ é regular. Por v) do Lema 2.8 temos que $X$ é um espaço de Lindelöf. Afirmamos que $X \times(\mathbb{R} \backslash \mathbb{Q})$ não é normal. Com efeito, mostremos que $X \times(\mathbb{R} \backslash \mathbb{Q})$ não é de Lindelöf, assim pelo Teorema 3.4 tal produto não será normal.

Consideremos o subconjunto de $X \times(\mathbb{R} \backslash \mathbb{Q})$

$$
A=\{(x, x): x \in Z\}
$$

Então $A$ é um subespaço fechado, discreto e não enumerável de $X \times(\mathbb{R} \backslash \mathbb{Q})$. Portanto não é de Lindelöf, o que implica que $X \times(\mathbb{R} \backslash \mathbb{Q})$ não pode ser de Lindelöf.

No que se segue, vamos apresentar um resultado de Lawrence [10] que permite encontrar espaços de Lindelöf cujo produto topológico com o espaço $\mathbb{R} \backslash \mathbb{Q}$ é normal e também de Lindelöf. Antes vejamos alguns resultados preliminares:

Lema 2.11. Seja $S$ um espaço métrico separável e $X$ um espaço de Lindelöf que é concentrado em um subconjunto fechado $A \subseteq X$ onde $A \times S$ é normal. Então as seguintes condições são equivalentes:

i. $X \times S$ é normal

ii. Para cada subconjunto não enumerável $B \subseteq X \backslash A$ e cada função injetora $F: B \longrightarrow S$, temos que:

$$
\operatorname{cl}(\operatorname{Graph}(F)) \cap(A \times S) \neq \phi
$$


Dem: i. $\rightarrow$ ii.] Suponhamos que ii. não é verdadeira, então existem $B \subseteq X \backslash A$ não enumerável e $F: B \longrightarrow S$ injetora tais que $\operatorname{cl}(\operatorname{Graph}(F)) \cap(A \times S)=\phi$.

Nestas condições temos que $\operatorname{cl}(\operatorname{Graph}(F))$ e $(A \times S)$ são dois subconjuntos fechados e disjuntos em $X \times S$. Vejamos que eles não podem ser separados por abertos disjuntos em $X \times S$.

Seja $U \subseteq X \times S$ um aberto qualquer tal que $A \times S \subseteq U$. Seja $D$ um subconjunto denso e enumerável em $S$. Definamos para cada $d \in D$ o seguinte subconjunto:

$$
Y_{d}=\{x \in X:(x, d) \notin U\}
$$

Afirmamos que $Y_{d}$ é enumerável para cada $d \in D$. De fato, dado que $A \times\{d\} \subseteq U$, temos que para cada $a \in A$, existe $Z_{a}$ vizinhança aberta de $a$ em $X$ e existe também $W_{a}$ vizinhança aberta de $d$ em $S$ tais que $(a, d) \in Z_{a} \times W_{a} \subseteq U$. Vamos ver que $Y_{d} \subseteq X \backslash \bigcup\left\{Z_{a}: a \in A\right\}$.

Seja $z \in Y_{d}$ e suponhamos que $z \in Z_{a}$ para algum $a \in A$. Logo $(z, d) \in Z_{a} \times W_{a} \subseteq U$ e $\operatorname{assim} z \notin Y_{d}$, que é um absurdo. Portanto $Y_{d} \subseteq X \backslash \bigcup\left\{Z_{a}: a \in A\right\}$.

Logo, como $X$ é concentrado em $A$ e $A \subseteq \bigcup\left\{Z_{a}: a \in A\right\}$, ocorre que $X \backslash \bigcup\left\{Z_{a}\right.$ : $a \in A\}$ é enumerável. Portanto, para cada $d \in D, Y_{d}$ é um subconjunto enumerável de $X$.

Agora, como $B$ é não enumerável, podemos tomar $x_{0} \in B \backslash \bigcup\left\{Y_{d}: d \in D\right\}$. Logo temos que $\left\{x_{0}\right\} \times D \subseteq U$ e portanto $\left(x_{0}, F\left(x_{0}\right)\right) \in \operatorname{cl}(U)$. Isto mostra que $\operatorname{cl}(\operatorname{Graph}(F))$ e $(A \times S)$ não podem ser separados por abertos disjuntos em $X \times S$.

ii. $\rightarrow$ i.] Observação: Pelo Teorema 3.4 temos que $A \times S$ é de Lindelöf. Assim se para cada recobrimento aberto $\mathcal{V}$ em $X \times S$ de $A \times S$ o conjunto

$$
\mathcal{L}=\{x \in X:\{x\} \times S \text { não é coberto por } \mathcal{V}\}
$$

é enumerável, então $X \times S$ é de Lindelöf (também normal). 
Suponhamos que $X \times S$ não é normal. Logo pela observação anterior, temos que existe um recobrimento $\mathcal{V}$ de $A \times S$ formado por abertos de $X \times S$ tais que o conjunto $\mathcal{L}$ não é enumerável.

Seja $U=\bigcup \mathcal{V}$. Logo $U$ é um aberto em $X \times S$ tal que $A \times S \subseteq U$ e $(X \times S) \backslash U$ intersecta um número não enumerável de seções verticais de $X \times S$.

Para cada $s \in S$ definamos

$$
Y_{s}=\{x \in X:(x, s) \notin U\}
$$

De modo análogo, à demonstração, em i. $\rightarrow$ ii., de que cada $Y_{d}$ era enumerável, podese mostrar que, para cada $s \in S$ o conjunto $Y_{s}$ é enumerável. Mas $Z=\bigcup\left\{Y_{s}: s \in S\right\}$ não é enumerável (pois contém o conjunto não enumerável $\{x \in X: \exists s \in S(x, s) \in$ $(X \times S) \backslash U\})$.

Por recursão transfinita, vamos definir duas funções $\sigma: \omega_{1} \longrightarrow Z$ e $\tau: \omega_{1} \longrightarrow S$ do seguinte modo:

1. Tomamos $\sigma(0) \in Y_{\tau(0)}$.

2. Seja $\delta$ um número ordinal tal que $\delta<\omega_{1}$ e suponhamos definidos, para cada $\alpha<$ $\delta, \sigma(\alpha)$ e $\tau(\alpha)$ tais que $\sigma(\alpha) \in Y_{\tau(\alpha)} \backslash \bigcup\left\{Y_{\tau(\beta)}: \beta<\alpha\right\}$. Pela não enumerabilidade de $Z$, existe $\tau(\delta) \in S$ tal que $Y_{\tau(\delta)} \backslash \bigcup\left\{Y_{\tau(\beta)}: \beta<\delta\right\} \neq \phi$. Logo existe $\sigma(\delta) \in$ $Y_{\tau(\delta)} \backslash \bigcup\left\{Y_{\tau(\beta)}: \beta<\delta\right\}$.

Nestas condições definimos

$$
\begin{aligned}
F: i m(\sigma) & \longrightarrow S \\
\sigma(\alpha) & \longmapsto \tau(\alpha) .
\end{aligned}
$$

Notemos que:

1. $i m(\sigma) \subseteq X \backslash A$ é não enumerável. 
2. F é injetora.

Então ii. implica que $c l(G r a p h(F)) \cap(A \times S) \neq \phi$.

Seja $(x, s) \in \operatorname{cl}(\operatorname{Graph}(F)) \cap(A \times S)$. Então $(x, s) \in A \times S \subseteq U$ e assim $U \cap$ $\operatorname{Graph}(F) \neq \phi$. Logo existe $\alpha<\omega_{1}$ tal que $(\sigma(\alpha), \tau(\alpha)) \in U$, que é absurdo pois $\sigma(\alpha) \in Y_{\tau(\alpha)}($ ou seja $(\sigma(\alpha), \tau(\alpha)) \notin U)$.

Portanto, $X \times S$ é normal.

Definição 2.3. Suponhamos que $S$ é um espaço métrico separável e $A \subseteq S$ onde $S$ é concentrado em $A$. Definimos $\mathbb{L}(S, A)$ como sendo o conjunto $S$ com a topologia dada por

$$
\{U \cup V: U \text { é aberto em } S \text { e } V \subseteq S \backslash A\}
$$

Esta topologia é um refinamento de Lindelöf da topologia de $S$.

Notemos que como $S$ é regular, então o espaço $\mathbb{L}(S, A)$ é também regular (Ver [[6], pág. 306, exemplo 5.1.22]).

Corolário 2.12. Suponhamos que $S$ é um espaço métrico separável $e A \subseteq S$, onde $S$ é concentrado em $A$ e $S \backslash A$ é não enumerável. Então $\mathbb{L}(S, A) \times(S \backslash A)$ não é normal (onde $S \backslash A$ tem a topologia de subespaço).

Dem: Vamos supor que $\mathbb{L}(S, A) \times(S \backslash A)$ é normal. Notemos que $S \backslash A$ é um espaço métrico separável, $\mathbb{L}(S, A)$ é um espaço de Lindelöf concentrado em $A$ ( $A$ é um subespaço fechado de $\mathbb{L}(S, A))$ e $A \times(S \backslash A)$ é normal (pelo Teorema 3.4). Assim estamos nas condições do Lema 2.11. Portanto para todo $B \subseteq \mathbb{L}(S, A) \backslash A$ não enumerável e para toda função $F: B \longrightarrow S \backslash A$ injetora, ocorre que $\operatorname{cl}(\operatorname{Graph}(F)) \cap(A \times(S \backslash A)) \neq \phi$. 
Mas se consideramos a aplicação identidade $i d: \mathbb{L}(S, A) \backslash A \longrightarrow S \backslash A$, temos que

$$
\begin{aligned}
\operatorname{cl}(\operatorname{Graph}(i d)) \cap(A \times(S \backslash A)) & =\operatorname{cl}(\{(x, x): x \in S \backslash A\}) \cap(A \times(S \backslash A)) \\
& =\{(x, x): x \in S \backslash A\} \cap(A \times(S \backslash A)) \\
& =\phi
\end{aligned}
$$

o que é absurdo.

Portanto, $\mathbb{L}(S, A) \times(S \backslash A)$ não é normal.

Lema 2.13. Seja $Y$ um espaço completamente metrizável e separável e $A \subseteq X \subseteq Y$, onde $X$ é concentrado em $A$ e $\mathbb{L}(X, A) \times(\mathbb{R} \backslash \mathbb{Q})$ não normal. Então existe um espaço de Cantor $K \subseteq Y$ tal que $K \cap A=\phi$ e $K \subseteq \operatorname{cl}_{Y}(A)$.

Dem: Vejamos as seguintes observações:

- Como $X$ é concentrado em $A$, então $\mathbb{L}(X, A)$ é concentrado em $A$.

- $A$ é fechado em $\mathbb{L}(X, A)$.

- $A \times(\mathbb{R} \backslash \mathbb{Q})$ é normal como subespaço de $\mathbb{L}(X, A) \times(\mathbb{R} \backslash \mathbb{Q})$, pois é de Lindelöf (Teorema 3.4). De fato, observemos que a topologia que herda $A$ como subespaço de $\mathbb{L}(X, A)$ é a mesma que herda como subespaço de $X$, o qual é hereditariamente separável (de Lindelöf), portanto $A \times(\mathbb{R} \backslash \mathbb{Q})$ vai ser um espaço de Lindelöf.

Das observações anteriores, como temos que $\mathbb{L}(X, A) \times(\mathbb{R} \backslash \mathbb{Q})$ não é normal, pelo Lema 2.11 existem $B \subseteq X \backslash A$ não enumerável e $F: B \longrightarrow \mathbb{R} \backslash \mathbb{Q}$ função injetora tal que

$$
c_{(\mathbb{L}(X, A) \times(\mathbb{R} \backslash \mathbb{Q}))}(\operatorname{Graph}(F)) \cap(A \times(\mathbb{R} \backslash \mathbb{Q}))=\phi
$$

Seja $G=c l_{Y \times(\mathbb{R} \backslash \mathbb{Q})}(G \operatorname{raph}(F))$, afirmamos que $G \cap(A \times(\mathbb{R} \backslash \mathbb{Q}))=\phi$.

De fato, seja $(a, p) \in A \times(\mathbb{R} \backslash \mathbb{Q})$, então por (2.3.2) existe $U$, vizinhança aberta de $(a, p)$ em $\mathbb{L}(X, A) \times(\mathbb{R} \backslash \mathbb{Q})$ tal que $U \cap \operatorname{Graph}(F)=\phi$. 
Observação: Podemos tomar $U$ aberto em $X \times(\mathbb{R} \backslash \mathbb{Q}$ ), dado que $a \in A$ (ver como foi definida a topologia de $\mathbb{L}(X, A))$.

Portanto, dada a observação anterior temos que existe $V$, aberto em $Y \times(\mathbb{R} \backslash \mathbb{Q})$, tal que

$$
U=V \cap(X \times(\mathbb{R} \backslash \mathbb{Q}))
$$

Logo segue que

$$
\phi=U \cap \operatorname{Graph}(F)=(V \cap(X \times(\mathbb{R} \backslash \mathbb{Q}))) \cap \operatorname{Graph}(F)=V \cap \operatorname{Graph}(F) .
$$

Assim mostramos que, para todo ponto $(a, p)$ de $A \times(\mathbb{R} \backslash \mathbb{Q})$, existe uma vizinahnça aberta $V_{(a, p)}$ em $Y \times(\mathbb{R} \backslash \mathbb{Q})$ tal que $V_{(a, p)} \cap G \operatorname{raph}(F)=\phi$. Portanto $G \cap(A \times(\mathbb{R} \backslash \mathbb{Q}))=\phi$.

Vamos construir agora conjuntos $\left\{K_{\varphi} \subseteq Y: \varphi \in\{0,1\}^{n}, n<\omega\right\}$ e $\left\{W_{\varphi} \subseteq \mathbb{R} \backslash \mathbb{Q}\right.$ : $\left.\varphi \in\{0,1\}^{n}, n<\omega\right\}$ tais que:

1. Para cada $n<\omega$ e para cada $\varphi \in\{0,1\}^{n}, K_{\varphi}$ seja aberto em $Y$ e $W_{\varphi}$ seja aberto em $\mathbb{R} \backslash \mathbb{Q}$.

2. $c l_{Y}\left(K_{\varphi_{0}, \ldots, \varphi_{n+1}}\right) \subseteq K_{\varphi_{0}, \ldots, \varphi_{n}} \quad$ e $\quad c l_{\mathbb{R} \backslash \mathbb{Q}}\left(W_{\varphi_{0}, \ldots, \varphi_{n+1}}\right) \subseteq W_{\varphi_{0}, \ldots, \varphi_{n}}$ para cada $n<\omega \mathrm{e}$ para cada $\varphi=\left(\varphi_{0}, \ldots, \varphi_{n+1}\right) \in\{0,1\}^{n+2}$.

3. $c l_{Y}\left(K_{0}\right) \cap c l_{Y}\left(K_{1}\right)=\phi \mathrm{e}$ $c l_{Y}\left(K_{\varphi_{0}, \ldots, \varphi_{n-1}, 0}\right) \cap c l_{Y}\left(K_{\varphi_{0}, \ldots, \varphi_{n-1}, 1}\right)=\phi$, para cada $n$ com $0<n<\omega$ e para $\varphi=$ $\left(\varphi_{0}, \ldots, \varphi_{n-1}\right) \in\{0,1\}^{n}$.

4. $c l_{\mathbb{R} \backslash \mathbb{Q}}\left(W_{0}\right) \cap c l_{\mathbb{R} \backslash \mathbb{Q}}\left(W_{1}\right)=\phi \mathrm{e}$ $c l_{\mathbb{R} \backslash \mathbb{Q}}\left(W_{\varphi_{0}, \ldots, \varphi_{n-1}, 0}\right) \cap c l_{\mathbb{R} \backslash \mathbb{Q}}\left(W_{\varphi_{0}, \ldots, \varphi_{n-1}, 1}\right)=\phi$, para cada $n$ com $0<n<\omega$ e para $\varphi=\left(\varphi_{0}, \ldots, \varphi_{n-1}\right) \in\{0,1\}^{n}$.

5. Para cada $n<\omega$, temos que $\operatorname{diam}\left(K_{\varphi_{0}, \ldots, \varphi_{n}}\right)<\frac{1}{n+1} \quad$ e $\operatorname{diam}\left(K_{\varphi_{0}, \ldots, \varphi_{n}} \times W_{\varphi_{0}, \ldots, \varphi_{n}}\right)<$ $\frac{1}{n+1}$.

6. Para cada $n<\omega$ e para cada $\varphi \in\{0,1\}^{n}$ ocorre $\left|\left(K_{\varphi} \times W_{\varphi}\right) \cap \operatorname{Graph}(F)\right| \geq \omega_{1}$. 
Para isso, como $|\operatorname{Graph}(F)| \geq \omega_{1}$, tomemos $t_{0}$ e $t_{1}$ diferentes em $B$ tais que $\left(t_{0}, F\left(t_{0}\right)\right)$ e $\left(t_{1}, F\left(t_{1}\right)\right)$ sejam pontos de condensação de $G r a p h(F)$ (para encontrar tais pontos escrevemos $\operatorname{Graph}(F)$ como união de dois conjuntos disjuntos não enumeráveis e aplicamos o Teorema 0.4).

Sejam $K_{0}$ e $K_{1}$ vizinhanças abertas em $Y$ de $t_{0}$ e $t_{1}$ respectivamente e $W_{0}$ e $W_{1}$ vizinhanças abertas em $\mathbb{R} \backslash \mathbb{Q}$ de $F\left(t_{0}\right)$ e $F\left(t_{1}\right)$ respectivamente, tais que:

- $c_{Y}\left(K_{0}\right) \cap c l_{Y}\left(K_{1}\right)=\phi$

- $\operatorname{diam}\left(K_{0}\right)<1, \operatorname{diam}\left(K_{1}\right)<1, \operatorname{diam}\left(K_{0} \times W_{0}\right)<1$ e $\operatorname{diam}\left(K_{1} \times W_{1}\right)<1$.

Suponhamos definidos agora $\left\{K_{\varphi} \subseteq Y: \varphi \in\{0,1\}^{n}\right\}$ e $\left\{W_{\varphi} \subseteq \mathbb{R} \backslash \mathbb{Q}: \varphi \in\{0,1\}^{n}\right\}$ satisfazendo as condições acima, para um certo $n$ fixo com $0<n<\omega$.

Seja $\varphi=\left(\varphi_{0}, \ldots, \varphi_{n-1}\right) \in\{0,1\}^{n}$.

Como $\left|K_{\varphi} \times W_{\varphi} \cap \operatorname{Graph}(F)\right| \geq \omega_{1}$, existem $t_{\varphi, 0}$ e $t_{\varphi, 1}$ em $B$ diferentes tais que $\left(t_{\varphi, 0}, F\left(t_{\varphi, 0}\right)\right)$ e $\left(t_{\varphi, 1}, F\left(t_{\varphi, 1}\right)\right)$ são pontos de condensação de $\left(K_{\varphi} \times W_{\varphi}\right) \cap \operatorname{Graph}(F)$. Tomemos $K_{\varphi_{0}, \ldots, \varphi_{n-1}, 0}$ e $K_{\varphi_{0}, \ldots, \varphi_{n-1}, 1}$ vizinhanças abertas em $Y$ de $t_{\varphi, 0}$ e $t_{\varphi, 1}$ respectivamente, e $W_{\varphi_{0}, \ldots, \varphi_{n-1}, 0}$ e $W_{\varphi_{0}, \ldots, \varphi_{n-1}, 1}$ vizinhanças abertas em $\mathbb{R} \backslash \mathbb{Q}$ de $F\left(t_{\varphi, 0}\right)$ e $F\left(t_{\varphi, 1}\right)$ respectivamente, tais que:

- $c l_{Y}\left(K_{\varphi_{0}, \ldots, \varphi_{n-1}, r}\right) \subseteq K_{\varphi_{0}, \ldots, \varphi_{n-1}}$ para $r=0,1$.

- $c l_{\mathbb{R} \backslash \mathbb{Q}}\left(W_{\varphi_{0}, \ldots, \varphi_{n-1}, r}\right) \subseteq W_{\varphi_{0}, \ldots, \varphi_{n-1}}$ para $r=0,1$.

- $c l_{Y}\left(K_{\varphi_{0}, \ldots, \varphi_{n-1}, 0}\right) \cap c l_{Y}\left(K_{\varphi_{0}, \ldots, \varphi_{n-1}, 1}\right)=\phi$.

- $c l_{\mathbb{R} \backslash \mathbb{Q}}\left(W_{\varphi_{0}, \ldots, \varphi_{n-1}, 0}\right) \cap c l_{\mathbb{R} \backslash \mathbb{Q}}\left(W_{\varphi_{0}, \ldots, \varphi_{n-1}, 1}\right)=\phi$.

- $\operatorname{diam}\left(K_{\varphi_{0}, \ldots, \varphi_{n-1}, r}\right)<\frac{1}{n+1}$ e $\operatorname{diam}\left(K_{\varphi_{0}, \ldots, \varphi_{n-1}, r} \times W_{\varphi_{0}, \ldots, \varphi_{n-1}, r}\right)<\frac{1}{n+1}$.

Portanto temos construído por recursão finita os conjuntos $\left\{K_{\varphi} \subseteq Y: \varphi \in\right.$ $\left.\{0,1\}^{n}, n<\omega\right\}$ e $\left\{W_{\varphi} \subseteq \mathbb{R} \backslash \mathbb{Q}: \varphi \in\{0,1\}^{n}, n<\omega\right\}$ com as condições que queríamos. 
Se consideramos as seguintes funções

$$
\begin{aligned}
\psi_{1}:\{0,1\}^{\omega} & \longrightarrow Y \\
\varphi & \longmapsto \psi_{1}(\varphi) \text { onde }\left\{\psi_{1}(\varphi)\right\}=\bigcap_{n=0}^{\infty} K_{\varphi_{0}, \ldots, \varphi_{n}} \\
\psi_{2}:\{0,1\}^{\omega} & \longrightarrow Y \times(\mathbb{R} \backslash \mathbb{Q}) \\
\varphi & \longmapsto \psi_{2}(\varphi) \quad \text { onde }\left\{\psi_{2}(\varphi)\right\}=\bigcap_{n=0}^{\infty} K_{\varphi_{0}, \ldots, \varphi_{n}} \times W_{\varphi_{0}, \ldots, \varphi_{n}}
\end{aligned}
$$

então temos os seguintes fatos:

- $H=\psi_{2}\left[\{0,1\}^{\omega}\right]$ e $K=\psi_{1}\left[\{0,1\}^{\omega}\right]$ são espaços de Cantor, (ver a demonstração do Teorema 1.13).

- $\prod_{1}[H]=K$, onde $\prod_{1}: Y \times(\mathbb{R} \backslash \mathbb{Q}) \longrightarrow Y$ é a projeção.

- $H \subseteq G$, pois senão, existiria $x \in H \backslash G$ e uma vizinhança aberta em $Y \times(\mathbb{R} \backslash \mathbb{Q})$ $U$ de $x$, tal que $U \subseteq(Y \times(\mathbb{R} \backslash \mathbb{Q})) \backslash G$. Tomemos $n$ suficientemente grande tal que exista $\varphi \in\{0,1\}^{n}$ tal que $x \in K_{\varphi} \times W_{\varphi} \subseteq U$. Então $\left(K_{\varphi} \times W_{\varphi}\right) \cap G=\phi$, o que é absurdo pois $\left|\left(K_{\varphi} \times W_{\varphi}\right) \cap G\right| \geq \omega_{1}$. Portanto $H \subseteq G$.

Afirmamos que $K \cap A=\phi$ e $K \subseteq c l_{Y}(A)$.

$K \cap A=\phi:$ Suponhamos que $K \cap A \neq \phi$, então existe $x \in K \cap A$. Seja $p \in \mathbb{R} \backslash \mathbb{Q}$ tal que $(x, p) \in H, \operatorname{logo}(x, p) \in H \cap(A \times(\mathbb{R} \backslash \mathbb{Q}))$, o que é absurdo, pois $H \subseteq G$ e $G \cap(A \times(\mathbb{R} \backslash \mathbb{Q}))=\phi$. Concluímos que $K \cap A=\phi$.

$K \subseteq \operatorname{cl}_{Y}(A):$ Seja $k \in K$ e tomemos $p \in \mathbb{R} \backslash \mathbb{Q}$ tal que $(k, p) \in H$. Consideremos $Z$ uma vizinhança aberta em $Y$ de $k$ e mostremos que $Z \cap A \neq \phi$.

De fato, se $W$ é uma vizinhança aberta em $\mathbb{R} \backslash \mathbb{Q}$ de $p$, então $(k, p) \in Z \times W$. Tomemos $n$ suficientemente grande tal que exista $\varphi \in\{0,1\}^{n}$ tal que $(k, p) \in K_{\varphi} \times W_{\varphi} \subseteq Z \times W$. Como $\left|\left(K_{\varphi} \times W_{\varphi}\right) \cap \operatorname{Graph}(F)\right| \geq \omega_{1}$ e $\operatorname{Graph}(F) \subseteq(X \backslash A) \times(\mathbb{R} \backslash \mathbb{Q})$ ocorre que $|Z \cap X| \geq \omega_{1}$. 
Suponhamos que $Z \cap A=\phi$. Dado que $|Z \cap X| \geq \omega_{1}$, pelo Teorema 0.4, existe $t^{\prime}$, ponto de condensação de $Z \cap X$. Tomemos $Z^{*}$ aberto em $Y$, tal que $t^{\prime} \in Z^{*} \subseteq \overline{Z^{*}} \subseteq Z$. Então $A \cap\left(\overline{Z^{*}} \cap X\right)=\phi$ e assim $A \subseteq X \backslash\left(\overline{Z^{*}} \cap X\right)$ (que é aberto em X), portanto $\overline{Z^{*}} \cap X$ é enumerável e assim $Z^{*} \cap X$ também seria enumerável, que é absurdo pois $\left|Z^{*} \cap X\right| \geq \omega_{1}$.

Mostramos que para cada $k \in K$ e para toda vizinhança aberta $Z_{k}$ em $Y$ desse ponto temos $Z_{k} \cap A \neq \phi$. Portanto $K \subseteq c l_{Y}(A)$.

Com isso terminamos a demonstração do teorema.

O teorema a seguir, fornece condição para o produto $\mathbb{L}(S, A) \times(\mathbb{R} \backslash \mathbb{Q})$ ser normal. Vejamos:

Teorema 2.14. Suponhamos que $Y$ é um espaço completamente metrizável e separável que é concentrado sobre um subconjunto $A$. Então $\mathbb{L}(Y, A) \times(\mathbb{R} \backslash \mathbb{Q})$ é normal.

Dem: Se $K$ é um espaço de Cantor contido em $Y$, então $K \cap A \neq \phi$. De fato, se $K \cap A=\phi$, teriamos que $Y \backslash K$ seria um aberto em $Y$ contendo o subconjunto $A$. Portanto $K$ seria um conjunto de Cantor enumerável, que é uma contradição. Portanto, $K \cap A \neq \phi$. Assim pela contrapositiva do Lema 2.13 , temos que $\mathbb{L}(Y, A) \times(\mathbb{R} \backslash \mathbb{Q})$ é normal. 


\section{Capítulo 3}

\section{Produtos com um fator Lindelöf e um fator métrico separável}

Neste capítulo, um dos nossos objetivos principais é mostrar um teorema provado por M. E. Rudin e M. Starbird ([16]), que apresentaremos no Teorema 3.4 (página 85). Ele foi suposto conhecido e usado em algumas demonstrações no capítulo anterior, por isso vamos demonstrá-lo agora.

É bom ressaltar que este mesmo teorema também é mostrado por A. Beslagic em [3], mas neste trabalho partindo das ideias dos matemáticos antes mencionados e junto com os resultados apresentados no apêndice $A$, damos uma demonstração que junta todas estas ideias.

Anteriormente no Capítulo 2, vimos exemplos de espaços normais tais que o produto com o subespaço topológico $\mathbb{R} \backslash \mathbb{Q}$ não era normal. Uma pergunta natural que podemos fazer é: quais são as condições necessárias para que esse produto seja normal. Vamos estudar um problema mais abrangente que responde essa pergunta parcialmente. Pensemos sob quais condições, para um espaço topológico $X$, as asserções " $X$ é normal" e $X \times Y$ é "normal para todo espaço métrico separável $Y$ " são equivalentes. Kiiti Morita (1964) deu uma resposta a esse problema (ver [14]) enunciando um teorema, que vamos demonstrar usando alguns resultados presentes nesse artigo e que neste trabalho vamos apenas enunciá-los. 


\subsection{Teorema de Rudin e Starbird}

Nesta seção os espaços métricos (ou metrizáveis) vão ser considerados não discretos, a menos que indiquemos o contrário, e todos os espaços topológicos serão supostos de Hausdorff.

O resultado seguinte se deve a Mary E. Rudin e Michael Starbird (ver [16]).

Teorema 3.1. Sejam $X$ um espaço normal e $(M, d)$ um espaço métrico. O produto topológico $X \times M$ é normal se e somente se $X \times M$ é enumeravelmente paracompacto.

Dem: Seja $\Pi_{1}: X \times M \longrightarrow X$ a função projeção.

$\Rightarrow$ ] Suponhamos que $X \times M$ seja um espaço normal.

Seja $\mathcal{B}=\bigcup\left\{\mathcal{B}_{n}: n<\omega\right\}$ uma base de $M$ tal que:

- Cada $\mathcal{B}_{n}$ é uma família localmente finita de abertos de $M$ com diâmetro menor que $1 / 2^{n}$.

- Cada elemento discreto de $\mathcal{B}$ contém exatamente um ponto.

Por recursão sobre os elementos não discretos de $\mathcal{B}$, podemos tomar pontos $p_{B} \neq q_{B}$ em $B$, para cada elemento não discreto $B \in \mathcal{B}$, tais que nenhum ponto de $M$ seja tomado duas vezes (ver Lema B.3, na página 101).

Para mostrar que $X \times M$ é enumeravelmente paracompacto, tomemos $\left\{U_{m}: m<\omega\right\}$ uma cobertura aberta qualquer de $X \times M$ e vamos construir uma cobertura aberta $\left\{V_{m, n}: m, n<\omega\right\}$ tal que $\overline{V_{m, n}} \subseteq U_{m}$ para cada $m, n<\omega$. Assim pelo Lema B.1 (na 
página 100), pode-se concluir que $X \times M$ é enumeravelmente paracompacto.

Para cada $B \in \mathcal{B}$ e $m<\omega$, seja $U_{B, m}$ o maior subconjunto aberto de $X$ tal que $U_{B, m} \times \bar{B} \subseteq U_{m}$. Definamos $F_{B}=X \backslash \bigcup\left\{U_{B, m}: m<\omega\right\}$. Notemos que cada $F_{B}$ é fechado em $X$ e que no caso de $B$ ser discreto, ocorre que $F_{B}=\phi$.

Tomemos $H_{B}=F_{B} \times\left\{p_{B}\right\}$ e $K_{B}=F_{B} \times\left\{q_{B}\right\}$ se $B$ não é discreto, caso contrário, definimos $H_{B}=F_{B}=\phi$. Para cada $n<\omega$, seja $H_{n}=\bigcup\left\{H_{B}: B \in \mathcal{B}_{n}\right\}$ e $K_{n}=$ $\bigcup\left\{K_{B}: B \in \mathcal{B}_{n}\right\}$.

Vamos verificar que para cada $n<\omega$, os conjuntos $\left\{H_{B}: B \in \mathcal{B}_{n}\right\}$ e $\left\{K_{B}: B \in \mathcal{B}_{n}\right\}$ são localmente finitos, e então teremos que $H_{n}$ e $K_{n}$ são conjuntos fechados. De fato, seja $(x, y) \in X \times M$. Dado que $\mathcal{B}_{n}$ é localmente finita, para $y$ existe $W_{y}$, vizinhança aberta de $y$ em $M$, tal que $W_{y}$ só intersecta $B_{n}^{(1)}, B_{n}^{(2)}, \ldots, B_{n}^{(r)}$ em $\mathcal{B}_{n}$, para algum $n<\omega$

Logo $X \times W_{y}$ é uma vizinhança aberta de $(x, y)$ tal que ela intersecta apenas um número finito de $H_{B}$ 's. Com efeito, se $B \notin\left\{B_{n}^{(1)}, B_{n}^{(2)}, \ldots, B_{n}^{(r)}\right\}$, então $H_{B} \cap\left(X \times W_{y}\right)=$ $\phi$, senão existiria $\left(z_{1}, z_{2}\right) \in H_{B} \cap\left(X \times W_{y}\right)$. Segue que $z_{2}=p_{B}$, e como $p_{B} \in B$, ocorreria que $W_{y} \cap B \neq \phi$, que é uma contradição, e portanto, $H_{B} \cap\left(X \times W_{y}\right)=\phi$. Assim $\left(X \times W_{y}\right)$ é uma vizinhança aberta de $(x, y)$ que intersecta um número finito de elementos $H_{B}$ 's. $\operatorname{Logo}\left\{H_{B}: B \in \mathcal{B}_{n}\right\}$ é localmente finita.

Analogamente, $\left\{K_{B}: B \in \mathcal{B}_{n}\right\}$ é localmente finita.

finamos $H=\bigcup\left\{H_{n}: n<\omega\right\}$ e $K=\bigcup\left\{K_{n}: n<\omega\right\}$. Notemos que:

1. $H \cap K=\phi$. Senão, existiria $(x, y) \in H \cap K$ o que implica que $(x, y) \in F_{B} \times\left\{p_{B}\right\}$ e $(x, y) \in F_{B^{*}} \times\left\{q_{B^{*}}\right\}$, para alguns $B \in \mathcal{B}_{k}$ e $B^{*} \in \mathcal{B}_{l}$. Então $p_{B}=q_{B^{*}}$ o qual não acontece. Portanto $H \cap K=\phi$.

2. $H$ e $K$ são conjuntos fechados em $X \times M$. De fato, fixemos um ponto $(x, y) \in$ $X \times M$ e encontremos um $k<\omega$ tal que $(x, y) \notin \overline{\bigcup\left\{H_{n}: n \geq k\right\}}$, isto mostra que $H$ é fechado. Analogamente $K$ também será fechado.

Sabemos que existe $m<\omega$ tal que $(x, y) \in U_{m}$. Logo existem também $B \subseteq M$ 
e $A \subseteq X$ abertos tais que $(x, y) \in A \times B \subseteq \bar{A} \times \bar{B} \subseteq U_{m}$. Seja $0<r<\omega$ tal que $S_{1 / 2^{r}}(y) \subseteq B$, e tomemos $k=2 r$ e $C=S_{1 / 2^{k}}(y)$. Vejamos que para cada $B^{*} \in \bigcup\left\{\mathcal{B}_{n}: n \geq k\right\}$ se $B^{*} \cap C \neq \phi$, então $B^{*} \subseteq B$. De fato, tomemos $B^{*}$ nessas condições $\left(B^{*} \in \mathcal{B}_{n}\right)$ e consideremos $w \in B^{*} \cap C$. Se $z \in B^{*}$ então $d(z, w)<1 / 2^{n}$, $\log \mathrm{O}$

$$
d(z, y) \leq d(z, w)+d(w, y)<\frac{1}{2^{n}}+\frac{1}{2^{k}} \leq 2\left(\frac{1}{2^{\min \{n, k\}}}\right)=\frac{1}{2^{k-1}}
$$

Como $k-1=2 r-1 \geq r$, segue-se que $d(z, y)<1 / 2^{r}$, ou seja, $z \in S_{1 / 2^{r}}(y) \subseteq B$. Portanto $B^{*} \subseteq B$.

Se mostrarmos que $(A \times C) \cap \bigcup\left\{H_{n}: n \geq k\right\}=\phi$, dado que $(x, y) \in A \times C$, podemos concluir que $(x, y) \notin \overline{\bigcup\left\{H_{n}: n \geq k\right\}}$. Neste sentido, se $\left(z_{1}, z_{2}\right) \in \bigcup\left\{H_{n}\right.$ : $n \geq k\}$, então existe $t<\omega$ tal que $\left(z_{1}, z_{2}\right) \in H_{t}$. Logo tomamos $B^{*} \in \mathcal{B}_{t}$ tal que $\left(z_{1}, z_{2}\right) \in H_{B^{*}}=F_{B^{*}} \times\left\{p_{B^{*}}\right\}, \operatorname{assim} z_{1} \in F_{B^{*}}$ e $z_{2}=p_{B^{*}}$.

Se $\left(z_{1}, z_{2}\right) \in A \times C$, dado que $z_{2}=p_{B^{*}} \in C \cap B^{*}$, temos que $B^{*} \subseteq B$. Logo, $\left(z_{1}, z_{2}\right) \in A \times \overline{B^{*}} \subseteq A \times \bar{B} \subseteq U_{m}$. Segue que $z_{1} \in U_{B^{*}, m}$ e então $z_{1} \notin F_{B}^{*}$, que é um absurdo. Portanto $(A \times C) \cap \bigcup\left\{H_{n}: n \geq k\right\}=\phi$.

Já que $X \times M$ é normal, consideremos $I$ e $J$ abertos em $X \times M$ tais que $H \subseteq I$, $K \subseteq J$ e $\bar{I} \cap \bar{J}=\phi$. Para cada $B \in \mathcal{B}$, seja $U_{B}=\Pi_{1}[I \cap(X \times B)] \cap \Pi_{1}[J \cap(X \times B)]$. Assim $U_{B}$ é aberto em $X$ e $F_{B} \subseteq U_{B}$. Portanto para cada $B \in \mathcal{B}$, a família $\left\{U_{B, m}: m<\right.$ $\omega\} \cup\left\{U_{B}\right\}$ é uma cobertura aberta de $X$. Dado que $M$ é não discreto, temos que $X$ é enumeravelmente paracompacto (ver o Lema B.4 no apêndice), e portanto podemos tomar uma cobertura aberta $\left\{V_{B, m}: m<\omega\right\} \cup\left\{V_{B}\right\}$ de $X$ tal que $\overline{V_{B, m}} \subseteq U_{B, m}$ e $\overline{V_{B}} \subseteq U_{B}$

Para cada $m, n<\omega$, definamos $V_{m, n}=\bigcup\left\{V_{B, m} \times B: B \in \mathcal{B}_{n}\right\}$. Como $\mathcal{B}_{n}$ é localmente finita, temos que $\overline{V_{m, n}}=\bigcup\left\{\overline{V_{B, m}} \times \bar{B}: B \in \mathcal{B}_{n}\right\} \subseteq U_{m}$. Falta mostrar que $\left\{V_{m, n}: m, n<\omega\right\}$ é um recobrimento de $X \times M$. Seja $(x, y) \in X \times M$ um ponto qualquer. Como $\bar{I} \cap \bar{J}=\phi$, então $(x, y) \notin \bar{I}$ ou $(x, y) \notin \bar{J}$. Sem perda de generalidade, suponhamos que $(x, y) \notin \bar{I}$. Fixemos $A \times B$ uma vizinhança aberta de $(x, y)$, com $B \in \mathcal{B}$ e $(A \times B) \cap \bar{I}=\phi$. Mas então $\Pi_{1}[I \cap(X \times B)] \cap A=\phi \operatorname{logo} x \notin U_{B}$ e portanto 
existe $m<\omega$ tal que $x \in V_{B, m}$. Segue-se que $(x, y) \in V_{B, m} \times B$, o que implica que $\left\{V_{m, n}: m, n<\omega\right\}$ é uma cobertura de $X \times M$.

Concluímos que $X \times M$ é enumeravelmente paracompacto.

$\Leftarrow]$ Suponhamos que $X \times M$ seja um espaço enumeravelmente paracompacto. Seja $\mathcal{G}=\left\{\mathcal{G}_{i}: i<\omega\right\}$ uma família de coberturas abertas localmente finitas de $M$ tais que:

(a) $\operatorname{diam}(G)<\frac{1}{2^{n}}$ para todo $G \in \mathcal{G}_{n}$.

(b) $\mathcal{G}_{n+1}$ é refinamento de $\mathcal{G}_{n}$ para cada $n<\omega$.

Para a existência de tal família veja o Lema B.2 (no apêndice página 101).

Sejam $H$ e $K$ dois fechados disjuntos em $X \times M$. Para cada $G \in \bigcup \mathcal{G}$ definamos

$$
R_{G}=\overline{\Pi_{1}[H \cap(X \times \bar{G})]} \cap \overline{\Pi_{1}[K \cap(X \times \bar{G})]}
$$

e para cada $n<\omega$ seja

$$
R_{n}=\bigcup\left\{R_{G} \times \bar{G}: G \in \mathcal{G}_{n}\right\}
$$

O conjunto $\left\{R_{n}: n<\omega\right\}$ tem as seguintes propriedades:

- $R_{n}$ é fechado em $X \times M$, para cada $n<\omega$, pois $\mathcal{G}_{n}$ é localmente finita.

- $R_{n+1} \subseteq R_{n}$. De fato, $(x, y) \in R_{n+1}$, existe $G \in \mathcal{G}_{n+1}$ tal que $(x, y) \in R_{G} \times \bar{G}$. Como $\mathcal{G}_{n+1}$ é refinamento de $\mathcal{G}_{n}$, existe $G^{*} \in \mathcal{G}_{n}$ tal que $G \subseteq G^{*}$, assim, $(x, y) \in$ $R_{G} \times \bar{G} \subseteq R_{G^{*}} \times \overline{G^{*}}$. $\operatorname{Logo}(x, y) \in R_{n}$. Portanto $R_{n+1} \subseteq R_{n}$.

- $\left\{\left\{R_{n}: n<\omega\right\}=\phi\right.$. Senão, existiria $(x, y) \in \bigcap\left\{R_{n}: n<\omega\right\}$. Logo para cada $n<\omega$ existiria $G_{n} \in \mathcal{G}_{n}$ tal que $x \in R_{G_{n}}$ e $y \in \overline{G_{n}}$. Vamos mostrar que isto implicaria que $(x, y) \in H \cap K$, o que é um absurdo.

De fato, consideremos $W \times V$ vizinhança aberta básica qualquer de $(x, y)$. Logo para cada $n<\omega$, temos que

$$
W \cap \Pi_{1}\left[H \cap\left(X \times \overline{G_{n}}\right)\right] \neq \phi \text { e } W \cap \Pi_{1}\left[K \cap\left(X \times \overline{G_{n}}\right)\right] \neq \phi .
$$


Sejam $w_{n}, z_{n} \in X$ tais que

$$
w_{n} \in W \cap \Pi_{1}\left[H \cap\left(X \times \overline{G_{n}}\right)\right] \text { e } z_{n} \in W \cap \Pi_{1}\left[K \cap\left(X \times \overline{G_{n}}\right)\right] .
$$

Logo existem $x_{n}, y_{n} \in M$ tais que $\left(w_{n}, x_{n}\right) \in H \cap\left(X \times \overline{G_{n}}\right)$ e $\left(z_{n}, y_{n}\right) \in K \cap(X \times$ $\left.\overline{G_{n}}\right)$.

Como $\operatorname{diam}\left(\overline{G_{n}}\right)<1 / 2^{n}, d\left(x_{n}, y\right)<1 / 2^{n}$ e $d\left(y_{n}, y\right)<1 / 2^{n}$. Portanto as sequências $\left(x_{n}\right)_{n \in \mathbb{N}}$ e $\left(y_{n}\right)_{n \in \mathbb{N}}$ convergem a $y$.

Dado que $V$ é uma vizinhança de $y$, existem $N_{1}, N_{2} \in \mathbb{N}$ tais que se $n \geq N_{1}$, então $x_{n} \in V$ e se $n \geq N_{2} \operatorname{logo} y_{n} \in V$. Tomemos $N_{0}=\max \left\{N_{1}, N_{2}\right\}$, assim se $n \geq N_{0}$, então $x_{n}, y_{n} \in V$.

Segue que $\left(w_{N_{0}}, x_{N_{0}}\right) \in(W \times V) \cap H$ e $\left(z_{N_{0}}, y_{N_{0}}\right) \in(W \times V) \cap K$. Concluímos então que $(x, y) \in \bar{H} \cap \bar{K}=H \cap K$.

Portanto, $\bigcap\left\{R_{n}: n<\omega\right\}=\phi$.

Nestas condições, pelo Teorema 0.11 , existe $\mathcal{S}=\left\{S_{n}: n<\omega\right\}$ cobertura aberta de $X \times M$ tal que para cada $n<\omega, \overline{S_{n}} \cap R_{n}=\phi$. Se tomamos para cada $n<\omega$, $U_{n}=\bigcup\left\{S_{k}: k \leq n\right\}$ então temos uma cobertura aberta de $X \times M$ tal que $\overline{U_{n}} \cap R_{n}=\phi$ e $U_{n} \subseteq U_{n+1}$ para cada $n<\omega$.

Fixe $n<\omega$. Definamos para cada $G \in \mathcal{G}_{n}$

$$
X_{G}=\left\{x \in X:(\{x\} \times G) \subseteq \overline{U_{n}}\right\} .
$$

Então os conjuntos $X_{G}$ e $R_{G}$, para cada $G \in \mathcal{G}_{n}$, são dois fechados disjuntos no espaço normal $X$. Portanto existe $V_{G} \subseteq X$ aberto tal que:

- $X_{G} \cap \overline{\Pi_{1}[H \cap(X \times \bar{G})]} \subseteq V_{G}$.

- $\overline{V_{G}} \cap \overline{\Pi_{1}[K \cap(X \times \bar{G})]}=\phi$.

Tomemos

$$
V_{n}=\bigcup\left\{V_{G} \times G: G \in \mathcal{G}_{n}\right\} .
$$

Deste modo concluímos que: 
1. $\overline{V_{n}} \cap K=\phi$ para cada $n<\omega$.

Senão, tomemos $\left(z_{1}, z_{2}\right) \in \overline{V_{n}} \cap K$. Então $\left(z_{1}, z_{2}\right) \in K$ e para cada vizinhança $W_{1} \times W_{2}$ de $\left(z_{1}, z_{2}\right)$ em $X \times M,\left(W_{1} \times W_{2}\right) \cap V_{n} \neq \phi$, ou seja existe $G \in \mathcal{G}_{n}$, tal que $\left(W_{1} \times W_{2}\right) \cap\left(V_{G} \times G\right) \neq \phi$.

Portanto, $W_{1} \cap V_{G} \neq \phi$ e $W_{2} \cap G \neq \phi$. Como $W_{1}$ e $W_{2}$ são vizinhanças quaisquer de $z_{1}$ e $z_{2}$ respectivamente, ocorre que $z_{1} \in \overline{V_{G}}$ e $z_{2} \in \bar{G}$. Assim $z_{1} \in \overline{V_{G}} \cap$ $\Pi_{1}[K \cap(X \times \bar{G})]$, o que é absurdo pela escolha dos $V_{G}$ 's. Portanto, $\overline{V_{n}} \cap K=\phi$.

2. $H \subseteq \bigcup\left\{V_{n}: n<\omega\right\}$.

Se $(x, y) \in H$, então existe $t<\omega$ tal que $(x, y) \in U_{t}$. Podemos tomar um $k<\omega$, suficientemente grande $(k \geq t)$ tal que $(x, y) \in\{x\} \times G_{k} \subseteq U_{t}$, para algum $G_{k} \in \mathcal{G}_{k} . \operatorname{Logo}\{x\} \times G_{k} \subseteq U_{k}$ (pois $U_{t} \subseteq U_{k}$ ), isto é, existe $G_{k} \in \mathcal{G}_{k}$ tal que $x \in X_{G_{k}}$.

Por outro lado, dado que $(x, y) \in H$ e $(x, y) \in X \times \overline{G_{k}}$, acontece que $x \in$ $\overline{\Pi_{1}\left[H \cap\left(X \times \bar{G}_{k}\right)\right]}$, segue que $x \in X_{G_{k}} \cap \overline{\Pi_{1}\left[H \cap\left(X \times \bar{G}_{k}\right)\right]} \subseteq V_{G_{k}}$. Portanto, dado que $y \in G_{k},(x, y) \in V_{k}$.

Concluímos que $H \subseteq \bigcup\left\{V_{n}: n<\omega\right\}$.

De modo análogo, podemos cobrir $K$ com um número enumerável de abertos, de tal modo que os seus fechos não intersectem $H$. Deste modo podemos concluir que $H$ e $K$ podem ser separados por abertos disjuntos em $X \times M$. Portanto, $X \times M$ é normal.

Corolário 3.2. Sejam $M$ um espaço metrizável e $K$ um espaço compacto. Se $X \times M$ e $X \times K$ são normais, então $X \times M \times K$ é também normal.

Dem: Pelo Teorema 3.1, temos que $X \times M$ é enumeravelmente paracompacto, então como $K$ é compacto, o produto $X \times M \times K=(X \times K) \times M$ é enumeravelmente paracompacto. Assim usando de novo o Teorema 3.1 concluímos que $(X \times M) \times K$ é normal. 
Corolário 3.3. Sejam $M$ um espaço metrizável e $X$ um espaço paracompacto. Se $X \times M$ é normal então $X \times M$ é paracompacto.

Dem: Tomemos $K=\beta(X \times M)$. Pelo Corolário 3.2, temos que o produto $X \times M \times K$ é normal. Portanto pelo Corolário A.5 (no apêndice), $X \times M$ é paracompacto.

Com os lemas, os corolários e os teoremas anteriores já temos as condições necessárias para apresentar o Teorema de Rundin e Starbird, e com ele chegamos a os nossos objetivos nesta seção. Lembremos que neste capítulo estamos supondo que os espaços métricos mencionados não são discretos, mas o teorema a seguir ainda vale se algum dos fatores for discreto, como é dito por Beslagic em [3, pág. 24]. Vejamos:

Teorema 3.4 (M. E. Rudin e M. Starbird). Sejam X um espaço regular de Lindelöf e Y um espaço metrizável e separável, então são equivalentes as seguintes condições:

i. $X \times Y$ é normal.

ii. $X \times Y$ é de Lindelöf.

Dem: i. $\Rightarrow$ ii.] Seja $D$ um subconjunto denso enumerável de $Y$. Então $X \times D$ é um subespaço denso de Lindelöf de $X \times Y$. Assim, se $X \times Y$ for paracompacto e $T_{2}$, então pelo Teorema $0.10, X \times Y$ será de Lindelöf. Claramente, $X \times Y$ é $T_{2}$. De fato, pelo Corolário 3.3, dado que $X$ é paracompacto (pois é regular e de Lindelöf), temos que $X \times Y$ é paracompacto. Portanto, $X \times Y$ é de Lindelöf.

ii. $\Rightarrow$ i.] Suponhamos que $X \times Y$ é um espaço de Lindelöf, então como ele é também regular (pois $X$ e $Y$ são regulares) temos que $X \times Y$ é um espaço normal. 


\subsection{Normalidade no produto de espaços normais com um espaço métrico separável}

Esta seção tem como objetivo responder a seguinte pergunta: Quais condições poderíamos exigir de um espaço normal $X$, para que o produto $X \times(\mathbb{R} \backslash \mathbb{Q})$ seja normal?

Como já falamos aqui, vamos tratar um problema um pouco mais abrangente. $\mathrm{K}$. Morita em [14], usando a noção de $P(2)$-espaços, conseguiu mostrar que se $X$ é um espaço topológico, então o produto de $X$ com qualquer espaço métrico separável é normal se e só se $X$ é um $P(2)$-espaço normal. Assim, claramente para todo $P(2)$ espaço normal o produto com o $\mathbb{R} \backslash \mathbb{Q}$ é normal.

Definição 3.1 ( $P(2)$-espaço). Seja $X$ um espaço topológico. Dizemos que $X$ é um $P(2)$-espaço se para cada família $\left\{G_{y_{0}, \ldots, y_{i}}: y_{i} \in\{0,1\}, i<\omega\right\}$ de subconjuntos abertos de $X$, tais que: $G_{y_{0}, \ldots, y_{i}} \subseteq G_{y_{0}, \ldots, y_{i}, y_{i+1}}$ para cada $i<\omega$, existe uma família $\left\{F_{y_{0}, \ldots, y_{i}}: y_{i} \in\{0,1\}, i<\omega\right\}$ de subconjuntos fechados de $X$ satisfazendo as seguintes condições

- $F_{y_{0}, \ldots, y_{i}} \subseteq G_{y_{0}, \ldots, y_{i}}$ para cada $y_{0}, y_{1}, . ., y_{i} \in\{0,1\}$ e para cada $i<\omega$.

- $X=\bigcup\left\{F_{y_{0}, \ldots, y_{i}}: i<\omega\right\}$ para cada sequência $y=\left(y_{0}, y_{1}, \ldots\right) \in\{0,1\}^{\omega}$ tal que $X=\bigcup\left\{G_{y_{0}, \ldots, y_{i}}: i<\omega\right\}$.

Exemplos de $P(2)$-espaços são espaços discretos, espaços normais enumeráveis e epaços métricos.

Os seguintes resultados estão demonstrados no artigo de Morita [14].

Lema 3.5. Sejam $X$ um $P(2)$-espaço normal e $S$ um subespaço topológico não vazio de $\{0,1\}^{\omega}$. Então o espaço topológico produto $X \times S$ é normal.

Lema 3.6. Seja $X$ um espaço topológico tal que para todo subespaço $S$ de $\{0,1\}^{\omega}$ o espaço topológico produto $X \times S$ é normal. Então $X$ é um $P(2)$-espaço normal. 
Teorema 3.7. Para que um espaço topológico X seja metrizável e separável é necessário e suficiente que exista um subespaço $S$ do espaço $\{0,1\}^{\omega}$ e uma função contínua, fechada e sobrejetora $g: S \longrightarrow X$ tal que $g^{-1}[\{x\}]$ seja compacto para cada ponto $x \in X$.

Teorema 3.8. Seja $X$ um espaço topológico. Para que $X \times Y$ seja normal para todo espaço métrico separável $Y$ é necessário e suficiente que $X$ seja um $P(2)$-espaço normal.

Dem: A condição necessária é consequência direta do Lema 3.6. Logo basta mostrar a condição suficiente.

Suponha que $X$ é um $P(2)$-espaço normal e $Y$ é um espaço métrico separável. Logo pelo Teorema 3.7, existem $S$ subespaço de $\{0,1\}^{\omega}$ e uma aplicação contínua, fechada e sobrejetora $g: S \longrightarrow Y$, tal que $g^{-1}[\{y\}]$ é compacto para cada ponto $y \in Y$. Assim,

$$
\begin{aligned}
f: X \times S & \longrightarrow X \times Y \\
(x, s) & \longmapsto f(x, s)=(x, g(s))
\end{aligned}
$$

é uma aplicação contínua, fechada e sobrejetora. Como pelo Lema 3.5, $X \times S$ é normal, temos que $X \times Y$ é também normal (pelo Teorema 0.5 ). 
Apêndices 


\section{Apêndice A}

\section{Produto com um fator compacto}

Nesta seção, estudaremos uma caracterização dada por Przymusiński em [15], baseada na noção de $\mathfrak{B}$-cobertura, para saber quando o produto de espaços normais com um fator compacto é normal. Para demonstrar o Teorema de Rudin e Starbird (Teorema 3.4), precisamos de alguns resultados que são consequência de tal caracterização.

Nesta seção vamos supor que todos os espaços mencionados são espaços de Hausdorff.

Lema A.1. Seja $X$ um espaço topológico $T_{4}$. Se $\mathcal{G}=\left\{G_{\theta}: \theta<\beta\right\}$ é uma cobertura aberta localmente finita de $X$ (onde $\beta$ é um ordinal), então existe $\mathcal{F}=\left\{F_{\theta}: \theta<\beta\right\}$ recobrimento fechado de $X$ tal que $F_{\theta} \subseteq G_{\theta}$ para cada $\theta<\beta$.

Dem: O conjunto $X \backslash \bigcup\left\{G_{\theta}: 0<\theta<\beta\right\}$ é um fechado em $X$ contido em $G_{0}$. Logo dado que $X$ é $T_{4}$, existe $Z_{0} \subseteq X$ aberto tal que

$$
X \backslash \bigcup\left\{G_{\theta}: 0<\theta<\beta\right\} \subseteq Z_{0} \subseteq \overline{Z_{0}} \subseteq G_{0}
$$

Notemos que $\left\{Z_{0}\right\} \cup\left\{G_{\theta}: 0<\theta<\beta\right\}$ é recobrimento aberto de $X$. Assim, $X \backslash\left(Z_{0} \cup \bigcup_{1<\theta<\beta} G_{\theta}\right)$ é um fechado em $X$ contido em $G_{1}$. Portanto, existe $Z_{1} \subseteq X$ 
aberto, tal que

$$
X \backslash\left(Z_{0} \cup \bigcup_{1<\theta<\beta} G_{\theta}\right) \subseteq Z_{1} \subseteq \overline{Z_{1}} \subseteq G_{1} .
$$

Por indução (finita ou transfinita dependendo se $\beta \leq \omega$ ou $\beta>\omega$ ) construímos o conjunto $\left\{Z_{\theta}: \theta<\beta\right\}$ como segue:

Suponhamos definidos para um ordinal $\alpha, \operatorname{com} \alpha<\beta$, o conjunto $\left\{Z_{\theta}: \theta<\alpha\right\}$ tal que $X \backslash\left(\bigcup_{\eta<\theta} Z_{\eta} \cup \bigcup_{\theta<\eta<\beta} G_{\eta}\right) \subseteq Z_{\theta} \subseteq \overline{Z_{\theta}} \subseteq G_{\theta}$ para cada $\theta<\alpha$ e $\left\{Z_{\theta}: \theta<\alpha\right\} \cup\left\{G_{\theta}\right.$ : $\alpha \leq \theta<\beta\}$ é cobertura aberta de $X$.

Assim, temos que $X \backslash\left(\bigcup_{\theta<\alpha} Z_{\theta} \cup \bigcup_{\alpha<\theta<\beta} G_{\theta}\right)$ é um fechado em $X$ contido em $G_{\alpha}$. Logo, dado que $X$ é $T_{4}$, existe $Z_{\alpha} \subseteq X$ aberto, tal que

$$
X \backslash\left(\bigcup_{\theta<\alpha} Z_{\theta} \cup \bigcup_{\alpha<\theta<\beta} G_{\theta}\right) \subseteq Z_{\alpha} \subseteq \overline{Z_{\alpha}} \subseteq G_{\alpha}
$$

e notemos que vale que $\left\{Z_{\theta}: \theta \leq \alpha\right\} \cup\left\{G_{\theta}: \alpha<\theta<\beta\right\}$ é recobrimento aberto de $X$.

Se definimos $F_{\theta}=\overline{Z_{\theta}}$ para cada $\theta<\beta$, temos o conjunto $\mathcal{F}=\left\{F_{\theta}: \theta<\beta\right\}$ como queríamos no lema. Claramente $F_{\theta} \subseteq G_{\theta}$ para cada $\theta<\beta$. Falta mostrar que $\mathcal{F}$ é cobertura de $X$. Para isto vamos considerar dois casos: se $\beta<\omega$ e se $\beta \geq \omega$.

Caso 1: Se $\beta<\omega$.

Se $\beta=0$ não temos nada que mostrar. Assim podemos considerar $0<\beta<\omega$. Então $\left\{Z_{0}, Z_{1}, \ldots, Z_{\beta-1}, G_{\beta}\right\}$ é cobertura aberta de $X$ e $X \backslash\left(Z_{0} \cup Z_{1} \cup \cdots \cup Z_{\beta-1}\right) \subseteq Z_{\beta} \subseteq \overline{Z_{\beta}} \subseteq$ $G_{\beta}$.

Seja $x \in X$. Se $x \in Z_{0} \cup Z_{1} \cup \cdots \cup Z_{\beta-1}$, então existe $n<\beta$ tal que $x \in F_{n}$. Caso contrário, $x \in X \backslash\left(Z_{0} \cup Z_{1} \cup \cdots \cup Z_{\beta-1}\right) \subseteq \overline{Z_{\beta}}=F_{\beta}$.

Portanto, $\left\{F_{0}, F_{1}, \ldots, F_{\beta}\right\}$ é cobertura fechada de $X$.

Caso 2: Se $\beta \geq \omega$. 
Tomemos $x \in X$ qualquer. Dado que $\mathcal{G}$ é localmente finita, sabemos que $x$ pertence a um número finito de elementos de $\mathcal{G}$. Seja $\alpha<\beta$ um ordinal tal que $x \notin G_{\theta}$ se $\alpha<\theta<\beta$ e $x \in G_{\alpha}$.

Se $x \in Z_{\theta}$ para algum $\theta, \operatorname{com} \theta<\alpha$, então $x \in F_{\theta}$. Senão, $x \in X \backslash\left(\bigcup_{\theta<\alpha} Z_{\theta} \cup\right.$ $\left.\bigcup_{\alpha<\theta<\beta} G_{\theta}\right) \subseteq Z_{\alpha} \subseteq \overline{Z_{\alpha}}$.

Portanto, $x \in F_{\alpha}$ e $\left\{F_{\theta}: \theta<\beta\right\}$ é uma cobertura fechada de X.

Observação: No que segue, se $K$ é um espaço compacto, vamos considerar sempre $\mathfrak{B}$ como sendo uma base de abertos do espaço $K$ fechada por uniões e intersecções finitas, isto é, se $A_{1}, \ldots, A_{n}$ são elementos de $\mathfrak{B}$, então $\bigcup_{i=1}^{n} A_{i} \in \mathfrak{B}$ e $\bigcap_{i=1}^{n} A_{i} \in \mathfrak{B}$.

Definição A.1. Sejam $\mathfrak{B}$ uma base de abertos de um espaço compacto $K$ e $(X, \tau)$ um espaço topológico. Seja também $H \subseteq \mathfrak{B} \times \mathfrak{B}$ o conjunto dado por:

$$
H=\{(B, D): B, D \in \mathfrak{B} \text { e } \bar{B} \cap \bar{D}=\phi\}
$$

e consideremos $G: H \longrightarrow \tau$ uma função. Dizemos que

$$
\mathfrak{G}=\{G(B, D):(B, D) \in H\}
$$

é uma $\mathfrak{B}$-cobertura de $X$ se $\mathfrak{G}$ é cobertura $e$

$$
G(B, D) \cap G\left(B^{*}, D^{*}\right)=G\left(B \cap B^{*}, D \cap D^{*}\right),
$$

para quaisquer $(B, D),\left(B^{*}, D^{*}\right) \in H$.

Observação: Se $B^{*} \subseteq B$ e $D^{*} \subseteq D$, então $G\left(B^{*}, D^{*}\right) \subseteq G(B, D)$.

Lema A.2. Sejam $\mathfrak{B}$ uma base de um espaço compacto $K e(X, \tau)$ um espaço topológico. Se $\mathcal{G}=\{G(B, D): B, D \in \mathfrak{B}$ e $\bar{B} \cap \bar{D}=\phi\}$ é uma $\mathfrak{B}$-cobertura de $X$ que tem um refinamento aberto localmente finito, então existe $\mathcal{V}=\{V(B, D): B, D \in \mathfrak{B}$ e 
$\bar{B} \cap \bar{D}=\phi\}$ refinamento aberto localmente finito de $X$ tal que $V(B, D) \subseteq G(B, D)$ para cada $B, D \in \mathfrak{B} \operatorname{com} \bar{B} \cap \bar{D}=\phi$.

Dem: Sejam $\left\{T_{i}: i \in I\right\}$ um refinamento aberto localmente finito de $\mathcal{G}$ e $H=$ $\{(B, D) \in \mathfrak{B} \times \mathfrak{B}: \bar{B} \cap \bar{D}=\phi\}$. Para cada $i \in I$, fixemos $f(i) \in H$ tal que $T_{i} \subseteq G(f(i))$. Temos definida uma função $f: I \longrightarrow H$.

Tomemos $\mathcal{V}=\{V(B, D):(B, D) \in H\}$ definida da seguinte maneira:

$$
V(B, D)= \begin{cases}\bigcup\left\{T_{k}: f(k)=(B, D)\right\} & \text { se }(B, D) \in i m f \\ \phi & \text { se }(B, D) \notin i m f\end{cases}
$$

Vemos que $\mathcal{V}$ é uma cobertura aberta de $X$, pois $\bigcup \mathcal{V}=\bigcup\left\{T_{i}: i \in I\right\}=X$. Além disso, $V(B, D) \subseteq G(B, D)$ para cada $(B, D) \in H$. Portanto para mostrar o lema basta ver que $\mathcal{V}$ é localmente finita. Para isso, seja $x \in X$. Dado que $\left\{T_{i}: i \in I\right\}$ é localmente finita, existe $W_{x} \subseteq X$, vizinhança aberta de $x$ em $X$, tal que o conjunto $I_{x}=\left\{i \in I: T_{i} \cap W_{x} \neq \phi\right\}$ é finito.

$\operatorname{Logo}\left\{(B, D) \in H: V(B, D) \cap W_{x} \neq \phi\right\} \subseteq\left\{f(i): i \in I_{x}\right\}$ é finito. Concluímos que $\mathcal{V}$ é localmente finita e portanto temos $\mathcal{V}$ como no lema.

Dada a definição e os lemas anteriores, podemos enunciar o seguinte teorema que é uma caracterização para a normalidade do produto de espaços topológicos com um fator compacto.

Teorema A.3 (Przymusiński). Seja $\mathfrak{B}$ uma base de um espaço compacto K. O espaço topológico $X \times K$ é normal se e só se $X$ é normal e qualquer $\mathfrak{B}$-cobertura de $X$ tem um refinamento aberto localmente finito.

Dem: $\Leftarrow]$ Suponhamos que $X$ é um espaço topológico normal e que qualquer $\mathfrak{B}$ cobertura de $X$ tenha um refinamento aberto localmente finito. Sejam $F$ e $L$ dois subconjuntos fechados de $X \times K$ tais que $F \cap L=\phi$.

Para cada $B, D$ em $\mathfrak{B}$ com $\bar{B} \cap \bar{D}=\phi$, definamos

$$
G(B, D)=\left\{x \in X: F_{x} \subseteq B \text { e } L_{x} \subseteq D\right\}
$$


onde

$$
F_{x}=\{y \in K:(x, y) \in F\}
$$

e

$$
L_{x}=\{y \in K:(x, y) \in L\} .
$$

Afirmação: $\mathfrak{G}=\{G(B, D): B, D \in \mathfrak{B}$ e $\bar{B} \cap \bar{D}=\phi\}$ é uma $\mathfrak{B}$-cobertura de $X$.

Para isto, observemos que dados $B, B^{*}, D, D^{*} \in \mathfrak{B}$ tais que $\bar{B} \cap \bar{D}$ e $\overline{B^{*}} \cap \overline{D^{*}}$ temos:

$$
\begin{aligned}
G\left(B \cap B^{*}, D \cap D^{*}\right) & =\left\{x \in X: F_{x} \subseteq B \cap B^{*} \text { e } L_{x} \subseteq D \cap D^{*}\right\} \\
& =\left\{x \in X: F_{x} \subseteq B \text { e } L_{x} \subseteq D\right\} \cap\left\{x \in X: F_{x} \subseteq B^{*} \text { e } L_{x} \subseteq D^{*}\right\} \\
& =G(B, D) \cap G\left(B^{*}, D^{*}\right) .
\end{aligned}
$$

Agora mostremos que $\mathfrak{G}$ cobre $X$. Seja $x \in X$ e lembremos que

$$
F_{x}=\{y \in K:(x, y) \in F\}
$$

e

$$
L_{x}=\{y \in K:(x, y) \in L\} .
$$

Dado que $F$ e $L$ são dois fechados disjuntos, então $F_{x}$ e $L_{x}$ são dois fechados disjuntos em $K$ e como este é compacto temos que $F_{x}$ e $L_{x}$ são também compactos.

Para cada $y \in F_{x}$ consideremos $B_{y}$ aberto básico que contém $y$ tal que $\overline{B_{y}} \cap L_{x}=\phi$. Então $\left\{B_{y}: y \in F_{x}\right\}$ é um recobrimento aberto de $F_{x}$, logo existem $y_{0}, y_{1}, \ldots, y_{n} \in F_{x}$ tais que $\left\{B_{y_{0}}, B_{y_{1}}, \ldots, B_{y_{n}}\right\}$ é um recobrimento finito de $F_{x}$. Portanto, podemos tomar $B \in \mathfrak{B}$ dado por

$$
B=B_{y_{0}} \cup B_{y_{1}} \cup \cdots \cup B_{y_{n}},
$$

aberto básico que contém $F_{x}$ e tal que $\bar{B} \cap L_{x}=\phi$. Agora, para cada $y \in L_{x}$ consideremos um aberto básico $D_{y}$ que contenha $y$ tal que $\overline{D_{y}} \cap \bar{B}=\phi$. Logo como $L_{x}$ é compacto, existe $\left\{D_{z_{0}}, D_{z_{1}}, \ldots, D_{z_{n}}\right\}$ sub-recobrimento finito da cobertura $\left\{D_{z}: z \in L_{x}\right\}$ de $L_{x}$. Tomando

$$
D=D_{z_{0}} \cup D_{z_{1}} \cup \cdots \cup D_{z_{n}}
$$


obtemos $D \in \mathfrak{B}$ que contém $L_{x}$ tal que $\bar{B} \cap \bar{D}=\phi$. Portanto $x \in G(B, D)$ como queríamos mostrar e assim $\mathfrak{G}$ é uma $\mathfrak{B}$-cobertura de $X$. Pela suposição no começo da demonstração, temos que $\mathfrak{G}$ tem um refinamento aberto localmente finito. Logo pelo Lema A.2, existe $\mathfrak{V}=\{V(B, D): B, D \in \mathfrak{B}$ e $\bar{B} \cap \bar{D}=\phi\}$ refinamento aberto localmente finito de $\mathfrak{G}$ tal que $V(B, D) \subseteq G(B, D)$. Além disso, dado que $X$ é um espaço normal, o Lema A.1 permite encontrar um refinamento fechado

$$
\mathfrak{Z}=\{Z(B, D): B, D \in \mathfrak{B} \text { e } \bar{B} \cap \bar{D}=\phi\}
$$

de $\mathfrak{V}$ tal que $Z(B, D) \subseteq V(B, D)$. Pela normalidade de $X$, existem funções contínuas $f_{B, D}: X \longrightarrow[0,1]$ tais que:

$$
f_{B, D}[Z(B, D)] \subseteq\{1\}
$$

e

$$
f_{B, D}[X \backslash V(B, D)] \subseteq\{0\}
$$

Agora, pela normalidade de $K$, temos que existem também funções contínuas $g_{B, D}$ : $K \longrightarrow[0,1]$ tais que:

$$
g_{B, D}[\bar{B}] \subseteq\{0\} \text { e } g_{B, D}[\bar{D}] \subseteq\{1\}
$$

Definamos $h: X \times K \longrightarrow \mathbb{R}$ como segue:

$$
h(x, y)=\sum\left\{f_{B, D}(x) \cdot g_{B, D}(y): B, D \in \mathfrak{B} \text { е } \bar{B} \cap \bar{D}=\phi .\right\}
$$

Como a família $\mathfrak{V}$ é localmente finita, $h$ está bem definida e é contínua.

Seja $(x, y) \in X \times K$, então $x$ pertence a um número finito de elementos de $\mathfrak{V}$. Digamos elementos $\left\{V\left(B_{i}, D_{i}\right): 0 \leq i \leq n\right\}$. Notemos que se $(B, D) \notin\left\{\left(B_{i}, D_{i}\right): 0 \leq\right.$ $i \leq n\}$, então $x \notin V(B, D)$ e assim $f_{B, D}(x)=0$. Portanto,

$$
h(x)=\sum_{i=0}^{n} f_{B_{i}, D_{i}}(x) g_{B_{i}, D_{i}}(y) .
$$

Se $(x, y) \in F$, temos que $y \in F_{x}$ e como $x \in G\left(B_{i}, D_{i}\right)$ para cada $i$ tal que $0 \leq i \leq n$, então $F_{x} \subseteq B_{i}$. Assim $g_{B_{i}, D_{i}}(y)=0$ se $0 \leq i \leq n$. Portanto, $h(x, y)=0$, o que mostra 
que $h[F] \subseteq\{0\}$

Se $(x, y) \in L$, temos que $y \in L_{x}$ e como $x \in G\left(B_{i}, D_{i}\right)$ para cada $i$ tal que $0 \leq i \leq n$, então $L_{x} \subseteq D_{i}$. Assim $g_{B_{i}, D_{i}}(y)=1$ se $0 \leq i \leq n$. Portanto, $h(x, y)=\sum_{i=0}^{n} f_{B_{i}, D_{i}}(x)$. Agora se $x \notin Z\left(B_{i}, D_{i}\right)$ para cada $0 \leq i \leq n$, então como $\mathfrak{Z}$ é cobertura de $X$, existiria $(B, D) \notin\left\{\left(B_{i}, D_{i}\right): 0 \leq i \leq n\right\}$ tal que $x \in Z(B, D) \subseteq V(B, D)$, que é absurdo pelo modo como o conjunto $\left\{\left(B_{i}, D_{i}\right): 0 \leq i \leq n\right\}$ foi escolhido. Portanto, $f_{B_{i}, D_{i}}(x)=1$ (pois $x \in Z\left(B_{i}, D_{i}\right)$ ) para algum $i$ com $0 \leq i \leq n$, ou seja $h(x, y) \geq 1$, que mostra $h[L] \subseteq[1, \infty[$.

Deste modo, $h^{-1}[]-\infty, 1 / 2[]$ e $h^{-1}[] 1 / 2, \infty[]$ são dois abertos disjuntos em $X \times K$ tais que $F \subseteq h^{-1}[]-\infty, 1 / 2[]$ e $L \subseteq h^{-1}[] 1 / 2, \infty[]$.

Portanto, $X \times K$ é normal.

$\Rightarrow] X$ é normal. Pois se $A$ é $B$ são dois fechados disjuntos em $X$, então $A \times K$ e $B \times K$ são dois fechados disjuntos em $X \times K$. Logo como $X \times K$ é normal, existem abertos $V_{A}$ e $V_{B}$ em $X$ tais que $A \times K \subseteq V_{A} \times K, B \times K \subseteq V_{B} \times K$ e $V_{A} \cap V_{B}=\phi$, o que mostra que $A$ e $B$ podem ser separados por abertos disjuntos em $X$.

Suponhamos agora que

$$
\mathfrak{G}=\{G(B, D): B, D \in \mathfrak{B} \text { e } \bar{B} \cap \bar{D}=\phi\}
$$

é uma $\mathfrak{B}$-cobertura de $X$. Definamos:

$$
\begin{aligned}
& F=(X \times K) \backslash \bigcup\{G(B, D) \times(K \backslash \bar{B}): B, D \in \mathfrak{B} \text { e } \bar{B} \cap \bar{D}=\phi\} \\
& L=(X \times K) \backslash \bigcup\{G(B, D) \times(K \backslash \bar{D}): B, D \in \mathfrak{B} \text { e } \bar{B} \cap \bar{D}=\phi\} .
\end{aligned}
$$

É claro que $F$ e $L$ são fechados em $X \times K$. Vejamos que são disjuntos:

Afirmação: $K \cap L=\phi$. 
Seja $(x, y) \in X \times K$, então existem $B$ e $D \in \mathfrak{B}$, com $\bar{B} \cap \bar{D}=\phi$, tais que $x \in G(B, D)$ e ademais $y \notin \bar{B}$ ou $y \notin \bar{D}$. Logo $(x, y) \notin F$ ou $(x, y) \notin L$ e portanto $K \cap L=\phi$.

Como $X$ é normal, pelo Lema de Urysohn, existe uma função contínua $f: X \times$ $K \longrightarrow[0,1]$ tal que $f[F] \subseteq\{0\}$ e $f[L] \subseteq\{1\}$.

Definamos uma pseudo-métrica $\rho$ em $X$ como segue:

$$
\rho\left(x, x^{\prime}\right)=\sup _{y \in K}\left|f(x, y)-f\left(x^{\prime}, y\right)\right|
$$

Observemos que $\rho$ está bem definida pois $K$ é compacto e contínua, pois é composição de funções contínuas.

Afirmação: $\mathfrak{G}$ tem um refinamento localmente finito.

Para cada $x_{0} \in X$ e cada $\varepsilon>0$ consideramos

$$
S\left(x_{0}, \varepsilon\right)=\left\{x \in X: \rho\left(x_{0}, x\right)<\varepsilon\right\}=\Pi_{2}\left[\rho^{-1}[]-\varepsilon, \varepsilon[] \cap\left(\left\{x_{0}\right\} \times X\right)\right]
$$

onde $\Pi_{2}: X \times X \longrightarrow X$ é a projeção na segunda coordenada. Dado que $\rho$ é contínua $\rho^{-1}[]-\varepsilon, \varepsilon[]$ é aberto em $X \times X$, portanto $\rho^{-1}[]-\varepsilon, \varepsilon[] \cap\left(\left\{x_{0}\right\} \times X\right)$ é um aberto em $\left\{x_{0}\right\} \times X$, e como $\Pi_{2}:\left\{x_{0}\right\} \times X \longrightarrow X$ é um homeomorfismo, obtemos que a topologia induzida por $\rho$ em $X$ está contida na topologia de $X$. Portanto para mostrar a afirmação é suficiente mostrar que a família $\{S(x, 1 / 3): x \in X\}$ é um refinamento aberto de $\mathfrak{G}$, pois pelo Teorema de Stone (ver [6, teorema 4.4.1] e o comentário posterior) essa cobertura tem um refinamento aberto localmente finito.

Fixemos $x_{0} \in X$; podemos encontrar $B, D \in \mathfrak{B}$ tais que

$$
\left\{y \in K: f\left(x_{0}, y\right) \leq 1 / 3\right\} \subseteq B \quad \text { e } \quad\left\{y \in K: f\left(x_{0}, y\right) \geq 2 / 3\right\} \subseteq D \quad \text { e } \quad \bar{B} \cap \bar{D}=\phi
$$

Vejamos que $S\left(x_{0}, 1 / 3\right) \subseteq G(B, D)$.

Se $x \in S\left(x_{0}, 1 / 3\right)$, então $F_{x} \subseteq B$ e $L_{x} \subseteq D$, senão poderia existir; por exemplo, um ponto $y \in F_{x} \backslash B$ e $f(x, y)=0, f\left(x_{0}, y\right)>1 / 3$ e $\rho\left(x_{0} . x\right)<1 / 3$, o que é absurdo. (analogamente para $L_{x} \backslash D$ ). 
Para mostrar que $x \in G(B, D)$, observemos que, pela definição de $F$ e $L$ e o fato acima, temos que:

$$
\begin{aligned}
& \{x\} \times(K \backslash B) \subseteq \bigcup\left\{G\left(B^{*}, D^{*}\right) \times\left(K \backslash \overline{B^{*}}\right): B^{*}, D^{*} \in \mathfrak{B} \text { e } \overline{B^{*}} \cap \overline{D^{*}}=\phi\right\} \\
& \{x\} \times(K \backslash D) \subseteq \bigcup\left\{G\left(B^{* *}, D^{* *}\right) \times\left(K \backslash \overline{D^{* *}}\right): B^{* *}, D^{* *} \in \mathfrak{B} \text { e } \overline{B^{* *}} \cap \overline{D^{* *}}=\phi\right\} .
\end{aligned}
$$

Então, pela compacidade desses conjuntos, existem famílias finitas $\left\{\left(B_{0}^{*}, D_{0}^{*}\right),\left(B_{1}^{*}, D_{1}^{*}\right), \ldots,\left(B_{n}^{*}, D_{n}^{*}\right)\right\}$ e $\left\{\left(B_{0}^{* *}, D_{0}^{* *}\right),\left(B_{1}^{* *}, D_{1}^{* *}\right), \ldots,\left(B_{m}^{* *}, D_{m}^{* *}\right)\right\}$ tais que:

$$
\begin{aligned}
& K \backslash B \subseteq \bigcup_{i=0}^{n} K \backslash \overline{B_{i}^{*}}, \quad K \backslash D \subseteq \bigcup_{j=0}^{m} K \backslash \overline{D_{j}^{* *}} \\
& x \in \bigcap_{i=0}^{n} G\left(B_{i}^{*}, D_{i}^{*}\right) \quad x \in \bigcap_{j=0}^{m} G\left(B_{i}^{* *}, D_{j}^{* *}\right) .
\end{aligned}
$$

Então

$$
\bigcap_{i=0}^{n} B_{i}^{*} \subseteq B \quad \bigcap_{j=0}^{m} D_{i}^{* *} \subseteq D
$$

e

$$
\begin{aligned}
x \in \bigcap_{i=0}^{n} G\left(B_{i}^{*}, D_{i}^{*}\right) \cap \bigcap_{j=0}^{m} G\left(B_{i}^{* *}, D_{i}^{* *}\right) & =G\left(\bigcap_{i=0}^{n} B_{i}^{*} \cap \bigcap_{j=0}^{m} B_{j}^{* *}, \bigcap_{i=0}^{n} D_{i}^{*} \cap \bigcap_{j=0}^{m} D_{j}^{* *}\right) \\
& \subseteq G(B, D) .
\end{aligned}
$$

O que mostra que $S\left(x_{0}, 1 / 3\right) \subseteq G(B, D)$. Portanto $\{S(x, 1 / 3): x \in X\}$ é um refinamento aberto de $\mathfrak{G}$, o que termina a demonstração.

Corolário A.4 (Morita). Sejam $K$ um espaço compacto e $X$ um espaço normal e $\omega(K)$-paracompacto, então $X \times K$ é normal.

Dem: Para poder aplicar o teorema anterior temos que mostrar que qualquer $\mathfrak{B}$ cobertura de $X$ admite um refinamento aberto localmente finito. Para isto, basta tomar uma base de $K$ de cardinalidade $\omega(K)$ e como por hipótese $X$ é $\omega(K)$-paracompacto, qualquer $\mathfrak{B}$-cobertura de $X$ tem um refinamento aberto localmente finito. Assim concluímos a tese do corolário. 
Corolário A.5 (Teorema de Tamano). As seguintes condições são equivalentes:

a) $X$ é paracompacto e $T_{2}$.

b) $X \times \beta X$ é normal.

Dem: a) $\Rightarrow$ b)] É claro pelo corolário anterior.

b) $\Rightarrow$ a)] Suponhamos que $X \times \beta X$ é normal. Como $X$ é normal, ele é $T_{2}$. Para a paracompacidade, seja $\mathcal{W}=\left\{W_{j}: j \in J\right\}$ uma cobertura aberta de $X$ e seja $\mathcal{U}=\left\{U_{j}: j \in J\right\}$ uma família de abertos de $\beta X$ tais que $W_{j}=X \cap U_{j}$ para cada $j \in J$. Consideremos $\mathfrak{B}$ como sendo a família de todos os subconjuntos abertos de $\beta X$ e definamos para $B, D \in \mathfrak{B}$

$$
G(B, D)=\left\{\begin{array}{ll}
B \cap X & \text { se } \beta X \backslash D \subseteq \cup \mathcal{U} \\
\phi & \text { caso contrário }
\end{array} .\right.
$$

Afirmação: $\mathfrak{G}=\{G(B, D): B, D \in \mathfrak{B}$ e $\bar{B} \cap \bar{D}=\phi\}$ é uma $\mathfrak{B}$-cobertura de $X$.

De fato, como

$$
G\left(B \cap B^{*}, D \cap D^{*}\right)=\left\{\begin{array}{ll}
\left(B \cap B^{*}\right) \cap X & \text { se } \beta X \backslash\left(D \cap D^{*}\right) \subseteq \cup \mathcal{U} \\
\phi & \text { caso contrário }
\end{array},\right.
$$

temos que $G\left(B \cap B^{*}, D \cap D^{*}\right)=G(B, D) \cap G\left(B^{*}, D^{*}\right)$. Falta mostrar que $\mathfrak{G}$ é uma cobertura de $X$. Para isto, seja $x \in X$. Dado que $\mathcal{U}$ é uma cobertura aberta de $X$, existe $U \in \mathcal{U}$ tal que $x \in U$. Portanto consideramos $B$, aberto de $\beta X$, tal que $x \in B \subseteq \bar{B} \subseteq U$ e $D$ um aberto em $\beta X$ tal que $\beta X \backslash U \subseteq D$ e $\bar{B} \cap \bar{D}=\phi$. Assim claramente $x \in G(B, D)$ o que mostra a afirmação.

Então, pelo Teorema A.3, temos que $\mathfrak{G}$ tem um refinamento $\mathcal{T}=\left\{T_{i}: i \in I\right\}$ aberto em $X$ localmente finito.

Observemos que o fecho em $\beta X$ de cada elemento de $\mathcal{T}$ está contido em $\bigcup \mathcal{U}$ (pela forma como foi definido cada $G(B, D)$ ), então é coberto por uma quantidade finita de 
elementos de $\mathcal{U}$. Suponhamos que para cada $i \in I, T_{i} \subseteq \bigcup\left\{U_{k}^{(i)}: 0 \leq k \leq n_{i}\right\}$. Seja $\mathcal{V}=\left\{T_{i} \cap U_{k}^{(i)}: i \in I\right.$ e $\left.0 \leq k \leq n_{i}\right\}$, então cada $T_{i} \cap U_{k}^{(i)}$ é aberto em $X$ contido em algum elemento de $\mathcal{W}$, pois $T_{i} \cap U_{k}^{(i)}=T_{i} \cap\left(U_{k}^{(i)} \cap X\right)=T_{i} \cap W_{k}^{(i)}$. Portanto $\mathcal{V}$ é um refinamento aberto de $\mathcal{W}$ localmente finito, o que mostra o corolário. 


\section{Apêndice B}

\section{Mais resultados usados no trabalho}

Lema B.1. Sejam $X$ um espaço topológico e $\mathcal{U}=\left\{U_{m}: m<\omega\right\}$ uma cobertura aberta de $X$ tal que existe $\mathcal{V}=\left\{V_{n, m}: n, m<\omega\right\}$, outra cobertura aberta de $X$ tal que $\overline{V_{m, n}} \subseteq U_{m}$ para cada $n, m<\omega$. Então existe um refinamento aberto localmente finito de $\mathcal{U}$.

Dem: Definamos para cada $n<\omega, W_{n}=U_{n} \backslash \bigcup\left\{\overline{V_{i, k}}: i, k<n\right\}$. Deste modo, o conjunto $\mathcal{W}=\left\{W_{n}: n<\omega\right\}$ é um refinamento aberto localmente finito de $\mathcal{U}$. De fato, para mostrar que $\mathcal{W}$ é cobertura de $X$, consideremos $x \in X$ qualquer, seja $m=\min \left\{n<\omega: x \in U_{n}\right\}$; temos $x \in W_{m}$. Portanto, $\mathcal{W}$ é uma cobertura aberta de $X$, mas como $W_{n} \subseteq U_{n}$ para cada $n<\omega$, então também é refinamento aberto de $\mathcal{U}$.

Para mostrar que $\mathcal{W}$ é localmente finito, consideremos de novo $x \in X$, qualquer, e $m=\min \left\{n<\omega: x \in U_{n}\right\}$. Como $\mathcal{V}$ é cobertura aberta de $X$, existem $k, l<\omega(k \geq m)$ tais que $x \in V_{k, l}$. Seja $t=\max \{k, l\}$, logo se $n>t$ temos que $W_{n} \cap V_{k, l}=\phi$. Portanto existe $V_{k, l}$, vizinhança aberta de $x$, que intersecta um número finito de elementos de $\mathcal{W}$, o que termina a demonstração do lema.

Rudin e Starbird apresentaram o seguinte lema em [16, fato 6], (eles se referem para a sua demonstração a [22]): 
Lema B.2. Se $M$ é um espaço métrico, então existe uma família $\mathcal{G}=\left\{\mathcal{G}_{i}: i<\omega\right\}$ de coberturas abertas localmente finitas de $M$ tais que:

(a) $G \in \mathcal{G}_{n}$ então $\operatorname{diam}(G)<\frac{1}{2^{n}}$.

(b) $\mathcal{G}_{n+1}$ é refinamento de $\mathcal{G}_{n}$ para cada $n<\omega$.

Lema B.3. Sejam $X$ um espaço métrico e $\mathcal{G}$ uma família de coberturas abertas localmente finitas como no Lema B.2. Se $\mathcal{G}_{n}^{\prime}=\left\{G \in \mathcal{G}_{n}: G\right.$ é não discreto $\}$, então para cada $G \in \mathcal{G}^{\prime}=\bigcup\left\{\mathcal{G}_{n}^{\prime}: n<\omega\right\}$ podemos selecionar dois pontos $p_{G}$ e $q_{G}$ em $G$ tais que nenhum ponto de $X$ seja selecionado duas vezes.

Dem: Vamos indexar, para cada $n<\omega$, os elementos de $\mathcal{G}_{n}^{\prime}$ da seguinte forma: $\mathcal{G}_{n}^{\prime}=$ $\left\{G_{(n, \alpha)}: \alpha<\lambda_{n}\right\}$ (onde $\lambda_{n}$ é um ordinal para cada $n<\omega$ ).

Nós selecionamos nossos dois pontos de cada $G_{(n, \alpha)}$, de acordo com a ordem lexicográfica $(n, \alpha)<(m, \beta)$ se e só se $n<m$, ou $n=m$ e $\alpha<\beta$. Suponhamos escolhidos os pontos distintos em cada $G_{(n, \alpha)}$ para cada $(n, \alpha)<(m, \beta)$. Desejamos encontrar dois novos pontos em $G_{(m, \beta)}$. Como $G_{(m, \beta)} \in \mathcal{G}_{m}^{\prime}$, ele tem um ponto de acumulação $w$, mas como $\mathcal{G}_{n}$ é localmente finito para cada $n \leq m$, existe uma vizinhaça $W$ de $w$ aberta em $X$ tal que $W$ só intersecta um número finito de elementos de $\bigcup\left\{\mathcal{G}_{n}: n \leq m\right\}$. Segue-se que temos escolhidos só um número finito de elementos de $W$. Mas dado que $\left|W \cap G_{m \beta}\right|$ não é finita, podemos tomar dois pontos $p_{G_{(m, \beta)}}$ e $q_{G_{(m, \beta)}}$ em $G_{(m, \beta)}$ tais que eles são diferentes de $p_{G_{(n, \alpha)}}$ e $q_{G_{(n, \alpha)}}$ para cada $(n, \alpha)<(m, \beta)$.

Lema B.4. Se $X$ é um espaço métrico não discreto ou um espaço compacto infinito, então $X \times Y$ normal implica que $Y$ é enumeravelmente paracompacto

Dem: (Ver [16], fact $1 \mathrm{~d})$ ) 


\section{Referências Bibliográficas}

[1] Alas O, Aurichi L. F, Junqueira L e Tall F. D.: Non-productively Lindelöf spaces and small cardinals, To appear in Houston Journal of Mathematics.

[2] Alster K.: The product of a Lindelöf space with the space of irrationals under Martin's Axiom, Proceedings of the American Mathematical Society, Vol 110, 543$547,1990$.

[3] Beslagic A.: Normality in products ${ }^{a}$, The work of Mary Ellen Rudin, Ed. Franklin D. Tall. Annals of the New York Academy of Sciences, Vol 705, 17-46, 1993.

[4] van Douwen E.: The integers and topology, Handbook of Set-theoretic topology. North Holland, Amsterdam, 111-167, 1984.

[5] Dowker C. H.: On countably paracompact space, Canadian Journal of Mathematics, Vol 3, 219-224, 1951.

[6] Engelking R.: General Topology, Sigma Series In Pure Mathematics, Vol 6, Heldermann Verlag, Berlin, 1989.

[7] Fernandez P.: Medida e integração, IMPA, 1976.

[8] Hechler S. H.: On a Ubiquitous Cardinal, Proceedings of the American Mathematical Society, Vol 52, 348-352, 1975.

[9] Kunen K.: Set Theory - An Introduction to Independence Proofs, Vol 102 of Studies in Logic and the Foundations of Mathematics. North Holland Publishing Company, Amsterdan, 1980. 
[10] Lawrence L. B.: The influence of a small cardinal on the product of a Lindelöf space and the irrationals, Proceedings of the American Mathematical Society, Vol 110, Num 2, 535-542, 1990.

[11] Michael E.: The product of a normal space and a metric space need not be normal, Proceedings of the American Mathematical Society, Vol 69, 375-376, 1963.

[12] Morita K.: Note on paracompactness, Proceedings of the Japan Academy, Vol 37, 1-3, 1961.

[13] Morita K.: Paracompactness and product spaces, Fundamenta Mathematicae, Vol 50, 223-236, 1962.

[14] Morita K.: Products of normal spaces with metric spaces, Mathematische Annalen, Vol 154, 365-382, 1964.

[15] Przymusinski T. C.: Products of normal spaces. Handbook of Set-theoretic Topology. North Holland, editado por K. Kunen e J. E. Vaughan, Amsterdam, 781-826, 1984 .

[16] Rudin M. E. e Starbird M.: Products with a metric factor, General Topology and its Applications, Vol 5, 235-248, 1975.

[17] Rudin M. E.: Countable paracompactness and Souslin's problem, Canadian Journal of Mathematics, Vol 7, 543-547, 1955.

[18] Rudin M. E.: к-Dowker space; Czechoslovak Mathematical Journal, Vol 28, 324$326,1978$.

[19] Sierpinski W.: Sur une propriété topologique des ensembles dénombrables denses en soi, Fundamenta Mathematicae, Vol. 1, 11-16, 1920.

[20] da Silva, Samuel Gomes: Uma introdução aos pequenos cardinais e às suas aplicações em topologia, Dissertação de mestrado apresentada ao IME da Universidade de São Paulo, 1998. 
[21] Solovay R. M. e S. Tennenbaum: Iterated Cohen extensions and Souslin's problem, Annals of Mathematics, Vol 94 (2), 201-245, 1971.

[22] Stone A. H.: Paracompactness and product spaces. Bulletin of the American Mathematical Society, Vol 54 977-982, 1948. 